\title{
Relativistic outflows from remnants of compact object mergers and their viability for short gamma-ray bursts
}

\author{
M. A. Aloy, H.-T. Janka, and E. Müller \\ Max-Planck-Institut für Astrophysik, Postfach 1317, 85741 Garching, Germany \\ e-mail: maa@mpa-garching.mpg.de
}

Received 19 August 2004 / Accepted 20 January 2005

\begin{abstract}
We present the first general relativistic hydrodynamic models of the launch and evolution of relativistic jets and winds, driven by thermal energy deposition, possibly due to neutrino-antineutrino annihilation, in the close vicinity of black hole-accretion torus systems. The latter are considered to be the remnants of compact object mergers. Our two-dimensional simulations establish the link between models of such mergers and future observations of short gamma-ray bursts by the SWIFT satellite. They show that ultrarelativistic outflow with maximum terminal Lorentz factors around 1000 develops for polar energy deposition rates above some $10^{48} \mathrm{erg} \mathrm{s}^{-1}$ per steradian, provided the merger environment has a sufficiently low baryon density. By the interaction with the dense accretion torus the ultrarelativistic outflow with Lorentz factors $\Gamma$ above 100 is collimated into a sharp-edged cone that is embedded laterally by a wind with steeply declining Lorentz factor. The typical semi-opening angles of the $\Gamma>100$ cone are $5^{\circ}-10^{\circ}$, corresponding to about $0.4-1.5 \%$ of the hemisphere and apparent isotropized energies (kinetic plus internal) up to $\approx 10^{51}$ erg although at most $10-30 \%$ of the deposited energy is transferred to the outflow with $\Gamma>100$. The viability of post-merger black hole-torus systems as engines of short, hard gamma-ray bursts is therefore confirmed. The annihilation of neutrino-antineutrino pairs radiated from the hot accretion torus appears as a suitable energy source for powerful axial outflow even if only $\approx 10^{49} \mathrm{erg}$ are deposited within a cone of $45^{\circ}$ half-opening angle around the system axis. Although the torus lifetimes are expected to be only between some $0.01 \mathrm{~s}$ and several $0.1 \mathrm{~s}$, our models can explain the durations of all observed short gamma-ray bursts, because different propagation velocities of the front and rear ends will lead to a radial stretching of the ultrarelativistic fireball before transparency is reached. The ultrarelativistic flow reveals a highly non-uniform structure caused by the action of Kelvin-Helmholtz instabilities that originate at the fireball-torus interface. Large radial variations of the baryon density (up to several orders of magnitude) are uncorrelated with moderate variations of the Lorentz factor (factors of a few) and fluctuations of the gently declining radiation-dominated pressure. In the angular direction the Lorentz factor reveals a nearly flat plateau-like maximum with values of several hundreds, that can be located up to $7^{\circ}$ off the symmetry axis, and a steep decrease to less than 10 for polar angles larger than $15^{\circ}-20^{\circ}$. Lateral expansion of the ultrarelativistic core of the flow is prevented by a subsonic velocity component of about $0.05 c$ towards the symmetry axis, whereas the moderately relativistic wings show a subsonic sideways inflation with less than $0.07 c$ (measured in the frame comoving with the radial flow).
\end{abstract}

Key words. gamma rays: bursts - hydrodynamics - methods: numerical - ISM: jets and outflows - shock waves

\section{Introduction}

Gamma-ray bursts (GRBs) are transient astrophysical events which are characterized by the release of enormous amounts of energy in non-thermal, highly variable (in time and event by event) emission on timescales between some milliseconds and about a thousand seconds. The distribution of burst durations reveals two classes, "short bursts" which last less than about $2 \mathrm{~s}$ and have typical duration of $0.3-0.5 \mathrm{~s}$, and "long bursts" with typically softer spectra and a mean durations of about 30-60 s (Kouveliotou et al. 1993).

So far only long bursts could be sufficiently well localized on the sky to enable multi-wavelength observations. In many such cases afterglow emission was detected, the host galaxies were discovered, and the associated measurement of spectral line redshifts confirmed the cosmological distances to the burst sources (e.g., Costa et al. 1997; Piro et al. 1998; van Paradijs et al. 1997; Bremer et al. 1998; Frail et al. 1997). Observational evidence also indicates that the bursts originate from star formation regions, suggesting a possible link to massive star explosions. This idea was nourished by several cases where a bump-like re-brightening phase of the decaying afterglow emission was interpreted as a superimposed supernova lightcurve. The link was directly confirmed by two cases where the spatial and temporal coincidence of a GRB with a peculiar kind of supernova explosion was found, GRB 980425 with SN 1998bw (Galama et al. 1998), which was still ambiguous, and GRB 030329 with SN 2003dh (Stanek et al. 2003; 
Hjorth et al. 2003). For short bursts such constraining observations do not exist.

According to the most widely accepted theoretical concept GRBs are understood as the consequence of an explosive release of energy associated with the accretion of matter at huge rates (up to a several solar masses per second) onto stellarmass black holes, e.g., as a consequence of the birth of such a black hole $(\mathrm{BH})$ by the catastrophic collapse of a massive star in a so-called "collapsar" event (Woosley 1993; MacFadyen \& Woosley 1999; Aloy et al. 2000; MacFadyen et al. 2001) or by a merger of compact objects in a close binary (Pacy ński 1986; Goodman 1986; Eichler et al. 1989; Mochkovitch et al. 1993). The nascent black hole is initially girded by a thick gas torus from which it swallows matter at a hypercritical rate, i.e., at a rate far in excess of the Eddington limit. In this case radiation is advected inward with the accretion flow and the cooling is dominated by the emission of neutrinos (Popham et al. 1999), a situation for which the term "neutrino-dominated accretion disk" (NDAF) was coined (Narayan et al. 2001). The annihilation of neutrino-antineutrino pairs in the immediate vicinity of the black hole-torus system is considered to create an $\mathrm{e}^{+} \mathrm{e}^{-}$-pair plasma-photon fireball which could power an ultrarelativistic outflow of baryons (with typical Lorentz factors $>10^{2}$ ) provided the baryon loading remains sufficiently low (Cavallo \& Rees 1978). Alternatively, magnetohydrodynamic processes might accelerate matter to ultrarelativistic velocities by tapping the gravitational binding energy of the accretion flow (e.g., Drenkhahn 2002; Drenkhahn \& Spruit 2002) or the rotational energy of the black hole (Blandford \& Znajek 1977). When these highly relativistic ejecta finally reach optically thin conditions at much larger radii $\left(\sim 10^{14} \mathrm{~cm}\right)$ the kinetic energy is dissipated and the observable GRB is produced mainly by synchrotron radiation and inverse Compton scattering (e.g., see the recent review by Mészáros 2002).

A large number of theoretical studies focused on the physics of the relativistic fireball and the formation of the observable properties of the GRB and of the afterglow emission (for reviews, see e.g., Mészáros 2002; Piran 1999), assuming that the energy release by some compact central engine has been able to produce the ultrarelativistic outflow. However, only few of the suggested astrophysical scenarios or concepts have so far been investigated by detailed numerical simulations which attempt to develop a quantitative picture of the physical processes that play a role at the source of the energy and that are responsible for driving the highly relativistic ejection of matter.

Hypercritical steady-state accretion from accretion disks and tori was first discussed with semi-analytic models by Popham et al. (1999), and later reconsidered by Narayan et al. (2001), Di Matteo et al. (2002), and Kohri \& Mineshige (2002). These works have brought a theoretical understanding of the conditions that are present in the vicinity of the accreting $\mathrm{BH}$ and which determine the efficiency of energy loss by neutrino emission and the efficiency of energy conversion by neutrinoantineutrino annihilation. To this end, three-dimensional hydrodynamic simulations were performed by Ruffert \& Janka (1999) and more recently by Setiawan et al. (2004) for the time-dependent accretion in $\mathrm{BH}$-tori systems as the remnants of neutron star-neutron star (NS+NS) and NS+BH mergers. In this case the torus is not fed by an external mass reservoir as in case of a collapsar, and the timescale of $\mathrm{BH}$ accretion is determined by viscous transport in the torus, not by the collapse timescale of a massive star. The torus is very compact. Typically it contains a few hundredth up to some 0.1 solar masses and extends to 15-20 Schwarzschild radii in the equatorial plane and about half that distance in the vertical direction. Its density and accretion rate are therefore high so that the torus can become partly opaque to neutrinos. With neutrino luminosities in excess of $10^{53} \mathrm{erg} \mathrm{s}^{-1} v \bar{v}$-annihilation can deposit energy in the close vicinity of the black hole at rates between several $10^{50} \mathrm{erg} \mathrm{s}^{-1}$ and more than $10^{51} \mathrm{erg} \mathrm{s}^{-1}$ (Ruffert \& Janka 1999; Janka et al. 1999).

The early phase of the propagation of jets in collapsars has been studied by nonrelativistic (MacFadyen \& Woosley 1999; MacFadyen et al. 2001) as well as special (Zhang \& MacFadyen 2003) or general relativistic (Aloy et al. 2000; Aloy \& Martí 2002) hydrodynamic simulations in two dimensions (i.e., assuming axial symmetry). Also first axisymmetric magnetohydrodynamic simulations have been done for the formation of polar outflows from the central non-rotating $\mathrm{BH}$ in a collapsar in both Newtonian (Proga et al. 2003; using a pseudoNewtonian potential, Pacyński \& Wiita 1980) and general relativity (Mizuno et al. 2004; assuming a fixed Schwarzshild metric). Numerical modeling of compact object mergers with some realism of the microphysics input (equation of state, neutrino processes) has made progress in case of NS+NS binaries (Ruffert et al. 1996a,b; Ruffert \& Janka 2001; Rosswog \& Liebendörfer 2003; Rosswog et al. 2003) and NS+BH systems (Janka et al. 1999; Janka \& Ruffert 2002; Rosswog et al. 2004), but detailed hydrodynamic simulations of the formation of the relativistic outflow from the accreting post-merging $\mathrm{BH}$ have not been undertaken prior to the present work.

In this paper we present results of the first general relativistic (a fix Schwarzschild metric is assumed), axisymmetric models for the acceleration and expansion of ultrarelativistic jets and winds, which originate from the pair-plasma-photon fireball that is produced by the deposition of thermal energy above the poles of a stellar-mass $\mathrm{BH}$ that accretes matter from a thick accretion torus as obtained in NS+NS or NS+BH merger simulations (Ruffert \& Janka 1999; Janka et al. 1999). We use the high-resolution shock-capturing code GENESIS (Aloy et al. $1999)$ to integrate the general relativistic hydrodynamic equations in "2.5 dimensions" (i.e., in spherical $(r, \theta)$ coordinates assuming that there is no azimuthal $(\phi)$ dependence of physical quantities although the $\phi$-components of vectors can have non-zero values).

Our simulations attempt to answer questions about the collimation mechanism of the polar outflow, the opening angle of the ultrarelativistic ejecta, the maximum and mean Lorentz factors that can be attained, the internal structure of the outflow, the duration of a possible GRB event, and the isotropic equivalent energy which an observer would infer by assuming the source to expand isotropically.

These questions may at most be guessed but they cannot be reliably answered on grounds of merger models and a consideration of their energy release by neutrino emission and the subsequent conversion of some of this energy by $v \bar{v}$-annihilation to 
$\mathrm{e}^{+} \mathrm{e}^{-}$-pairs (Ruffert \& Janka 1999; Janka et al. 1999; Rosswog \& Ramirez-Ruiz 2002; Rosswog et al. 2003). We stress here that the fraction of the latter energy which ends up in ultrarelativistic outflow with low baryon loading cannot be deduced or "extrapolated" from known spatial distributions of mass and $v \bar{v}$-energy deposition at any instant of a merger or accretion simulation without self-consistently time-dependent hydrodynamic modeling. The relativistic outflow develops in a complex hydrodynamic interaction with the accretion torus, cleaning its own axial funnel such that later energy deposition encounters a much reduced baryon pollution.

Answers to the questions mentioned above are of crucial importance for discussing compact binary mergers as potential sources of observed short GRBs and the observational properties of those. Current work on these issues (e.g., Rosswog \& Ramirez-Ruiz 2003) is much handicapped by the lack of quantitative information from detailed numerical simulations. Conclusions in the latter paper, for example, depend sensitively on the collimation of the ultrarelativistic outflow. In this context work by Levinson \& Eichler (2000) is referred to, who suggest a hydrodynamic collimation of the relativistic fireball by a surrounding baryonic wind emanating from the accretion torus and calculate the corresponding opening angle by analytic solutions. The discussion by these authors, however, is based on a number of simplifications and assumptions and therefore awaits verification by more complete models. Our simulations, for example, reveal that the vertical extension of the thick accretion torus cannot be ignored but plays a decisive role with respect to outflow collimation, baryon entrainment and structure development by Kelvin-Helmholtz instabilities at the jettorus interface.

Our paper is organized as follows. In Sect. 2 we shall describe in some more detail our model setup, the initial conditions, the numerical methods, and the parameters and assumptions which are varied systematically in our studies. Section 3 will provide a discussion of our results for two types of model sequences with different assumptions about the conditions for the deposition of thermal energy in the vicinity of the BH-torus system. We distinguish "type-A" models (in Sect. 3.1) with fairly high density in the surroundings of the torus, and "type-B" models with low environmental density (Sect. 3.2). In Sect. 4 we shall also consider the evolution of type-B models for a period of $0.5 \mathrm{~s}$ after the onset of the energy release. In some of these models the energy deposition was taken constant up to $0.1 \mathrm{~s}$ and then switched off. In other models the energy deposition was modeled more "burst-like", reaching maximum values for a period of only 20 milliseconds and then decaying with time like a $t^{-3 / 2}$ power law (cf., Setiawan et al. 2004) or modulated during the whole evolution (including the decay phase) with a certain time variability of the energy released per unit of time. A summary of our main results and conclusions will follow in Sect. 5.

\section{The model}

Short GRBs (or, at least, a subclass of these events) might be produced by the systems resulting from mergers of pairs of compact objects (either two neutron stars or a neutron star and a black hole). The situation that can arise from the mentioned merging processes consists of a central black hole of a few solar masses girded by a thick accretion torus with mass between 0.05 and $\approx 0.3 M_{\odot}$ (see, e.g., Ruffert \& Janka 1999; Janka et al. 1999). Once the thick disk has formed, neutrino-antineutrino annihilation may release up to $\sim 10^{51} \mathrm{erg}$ above the poles of the black hole in a region that contains less than $10^{-5} M_{\odot}$ of baryonic matter. This may lead to the acceleration of this matter to ultrarelativistic speeds, possibly accounting for a GRB with observed properties. If the duration of the event is related to the lifetime of the system (Sari \& Piran 1997) this kind of events can only belong to the class of short GRBs because the expected timescale on which the black hole engulfs the disk is at most of a few hundred milliseconds (Ruffert \& Janka 1999; Janka et al. 1999; Lee \& Ramirez-Ruiz 2002; Setiawan et al. 2004).

In order to test the viability of post-neutron star merger systems as likely progenitors of short GRBs we have used general relativistic, hydrodynamic simulations for studying the formation, acceleration and early propagation of the relativistic outflow that is driven by the deposition of thermal energy near the accretion torus around a stellar-mass black hole. In such a black hole-torus configuration a jet is expected to be launched by any process which gives rise to a local deposition of energy and/or momentum, e.g., $v \bar{v}$-annihilation, or magnetohydrodynamic processes. We mimic this process by releasing thermal energy in a prescribed cone around the rotational axis of the system.

The initial model is set up to reproduce the expected state of a post-neutron star merger accretion torus around a central black hole with a mass in the range $2.44 M_{\odot}-3 M_{\odot}$. Thus, the initial model is not the result of a merger simulation of two compact objects (at least one of them being a neutron star) but instead, we use two different ad hoc procedures to construct it. In the first one, a toroidal distribution of matter and angular momentum is placed in our computational domain, embedded by a larger, essentially spherically symmetric dilute gas cloud. Such a configuration is suggested by the previous results of Ruffert \& Janka (1999) and Janka et al. (1999) where the gas cloud is produced by mass shed off the hot massive merger remnant. Of course, a more refined setup would require to take into account asphericities due to the angular momentum in the system. For the time being, however, we constrain ourselves to spherically symmetric external gas clouds and avoid further refinements. This initial configuration is not in equilibrium and we need to evolve it for some tenths of a second in order to obtain a relaxed initial state. The relaxation of the model leads to the shedding of mass from the torus that expands into the environment. Hence, the relaxed model has a relatively high environmental density of $\simeq 10^{2} \mathrm{~g} / \mathrm{cm}^{-3}$ (Fig. 3) between $r \simeq 10^{7} \mathrm{~cm}$ and $r \simeq 10^{9} \mathrm{~cm}$, declining steeply down to $\simeq 2.5 \times 10^{-3} \mathrm{~g} / \mathrm{cm}^{-3}$ at $r \simeq 3 \times 10^{9} \mathrm{~cm}$. We will refer to this kind of initial models as type-A models (Fig. 1). The torus and black hole masses of type-A models are $0.17 M_{\odot}$ and $3 M_{\odot}$ after the relaxation time, respectively. The inner grid boundary is located in type-A models at 4 gravitational radii $\left(R_{\mathrm{g}}=G M / c^{2} ; G, M\right.$ and $c$ being the gravitational constant, the mass of the black hole and the speed 
of light in vacuum, respectively), i.e., at $\approx 1.78 \times 10^{6} \mathrm{~cm}$ from the center.

The production of type-B initial models uses a prescription very close to that of Font \& Daigne (2002) in order to build an equilibrium torus around a Schwarzschild black hole. Differences with respect to Font \& Daigne (2002) arise from the fact that we do not use a barotropic equation of state (EoS) and, therefore, it is not possible to obtain from the specific enthalpy distribution (which is the primary thermodynamical variable that is obtained from the Euler equation) unambiguously all other thermodynamic variables in the equilibrium torus. Thus, the adiabatic index $(\gamma)$ at every point has to be guessed, i.e., the initial model is not in perfect equilibrium (although the initial state is much closer to equilibrium than in the previous case), and it also requires some transient period until the initial model evolves to a relaxed configuration. However, as the deviation from equilibrium is small we can start releasing energy right from the beginning of the simulation because whatever the changes due to the relaxation are, they are relatively small and they propagate radially at a speed smaller than any ultrarelativistic outflow. On the other hand, a neutron star merger remnant originates from a violent event and, therefore, both the emergent accretion torus and its environment must naturally be expected to exhibit some variability in time. Thus, the initial models of type-B, although not fully relaxed, are not in obvious conflict with the situation that is expected from merger simulations (Fig. 2). The type-B models have a much smaller environmental density than those of typeA for radii larger than $\approx 10^{7} \mathrm{~cm}$ and their density gradient (in most of the computational domain) is also steeper than in the other case $\left(\rho \sim r^{-3.4}\right.$; Fig. 3$)$. Models of type-B have a torus and a black hole mass of $0.13 M_{\odot}$ and $2.44 M_{\odot}$, respectively. The inner grid radial boundary is located in type-B models at $2 R_{\mathrm{g}}$, i.e., at $\approx 7.57 \times 10^{5} \mathrm{~cm}$ from the center.

\subsection{Numerical details}

In the radial direction the region of energy deposition extends from the inner to outer grid boundaries. In the angular direction, the half-opening angle of the deposition cone $\left(\theta_{0}\right)$ around the system axis spans a range which was varied between $30^{\circ}$ and $75^{\circ}$. From the results of Ruffert \& Janka (1999) and Janka et al. (1999) we have inferred a power-law decline for the energy deposition rate per unit of volume by $v \bar{v}$-annihilation in the direction perpendicular to the equatorial plane of the accretion torus. We have chosen an explicit form of the energy deposition law in terms of observer frame coordinates as

$\dot{q}=\dot{q}_{0}\left(z_{0} / z\right)^{n}$,

where $z$ is the distance along the rotation axis, $n$ is the powerlaw index, $z_{0}=2 R_{\mathrm{g}}$ is the minimum vertical distance above the equator where energy is deposited, and $\dot{q}_{0}$ is the normalization factor that we use to set the total energy deposition rate $\dot{E}$ as measured by a static observer at infinity. In this paper we have considered only the case $n=5$, because this value complies best with the data in Janka et al. (1999). We assume energy deposition rates which vary in the range from $10^{49} \mathrm{erg} \mathrm{s}^{-1}$ to $5 \times 10^{51} \mathrm{erg} \mathrm{s}^{-1}$ (for the summed values of both hemispheres) and which are constant in time. We have also included two cases where the energy release is chosen to be time dependent (the explicit time dependence of models B07 and B08 is provided in Eqs. (10) and (11), respectively). The considered values roughly bracket the expected energy deposition rates from $v \bar{v}$-annihilation in the context of post-neutron star merger accretion tori.

The simulations were performed with the multidimensional relativistic hydrodynamic code GENESIS (Aloy et al. 1999) using 2D spherical coordinates $(r, \theta)$. We assume equatorial symmetry and we cover the $\theta$-direction with 200 uniform zones between $0^{\circ}$ and $90^{\circ}$. In the $r$-direction the computational grid consists of 400 or 500 zones. These are spaced logarithmically between the inner boundary located at $4 R_{\mathrm{g}}$ (type-A models) or $2 R_{\mathrm{g}}$ (type-B models) and an outermost radius of $R_{\max }=$ $3 \times 10^{9} \mathrm{~cm}$ (type-A models) or $R_{\max }=2 \times 10^{10} \mathrm{~cm}$ (type-B models). Even for this moderate resolution, the typical number of time steps per model in order to reach a time of $0.5 \mathrm{~s}$ is $\approx 10^{6-7}$. Models with a larger number of radial zones were computed to verify the accuracy of our results (see Appendix A). From the comparison of these test runs it can be concluded that 500 radial zones are sufficient to exclude major influence of the numerical resolution on our results.

The first set of models (type-A), i.e., those having the smaller $R_{\max }$, is employed to study only the generation phase of a relativistic fireball by following the evolution of the resulting outflow in a medium of relatively high density up to $100 \mathrm{~ms}$. The second set of models, i.e., those having the larger $R_{\max }$, start from non-relaxed type-B initial models and, as the computational domain extends up to a larger radius, allows us to study the evolution of the fireballs in relatively low-density environments until $\sim 0.5 \mathrm{~s}$. We have also extended the domain of one of the most energetic type-A models in order to follow the evolution of fireballs in relatively high-density environments until $\sim 0.4 \mathrm{~s}$.

The space-time around the non-rotating black hole is described by the steady spherical Schwarzschild metric. Effects on the dynamics due to the self-gravity of the accretion torus or the external gas are neglected, i.e., we consider only the gravitational potential of the black hole. The values of the energy deposition rate that we will use throughout the paper are measured by an observer at infinity. Therefore, the energy deposition rate per unit of coordinate volume $\dot{q}$ measured by such an observer has to be blue-shifted in order to determine the local value of the energy deposition rate in the close vicinity of the BH. This value has to be further transformed to the local comoving fluid frame because it is numerically included in the algorithm as a source of the comoving energy density. The amount of energy released per unit of coordinate time and proper volume in the comoving frame is

$$
\dot{q}^{\prime}=\frac{\dot{q}}{\left(1-2 R_{\mathrm{g}} / r\right)^{1 / 2}\left(\gamma\left(\Gamma^{2}-1\right)+1\right)},
$$

where $\Gamma$ is the bulk Lorentz factor of the moving fluid.

The EoS assumes nuclei to be disintegrated to free, non-relativistic nucleons, treated as a mixture of Boltzmann gases, and includes the contribution from radiation, and an approximate correction due to $e^{+} e^{-}$-pairs as described in 


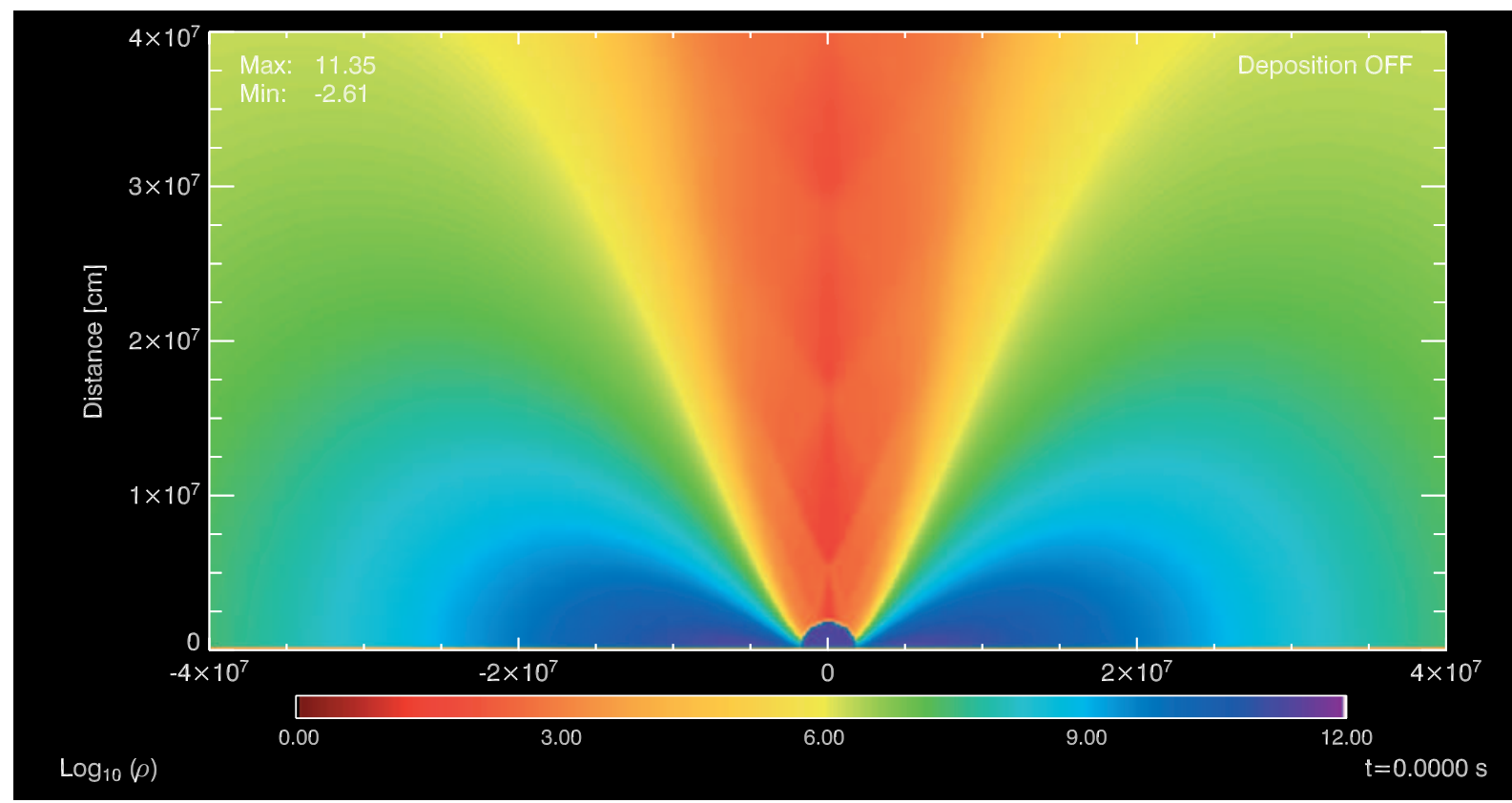

Fig. 1. Logarithm of the rest-mass density of the initial model of type-A simulations showing the structure of the relaxed accretion torus. The scale of the plot is limited in order to be able to distinguish properly the toroidal structure. In the upper left corner the maximum and minimum values of the displayed variable are reported.

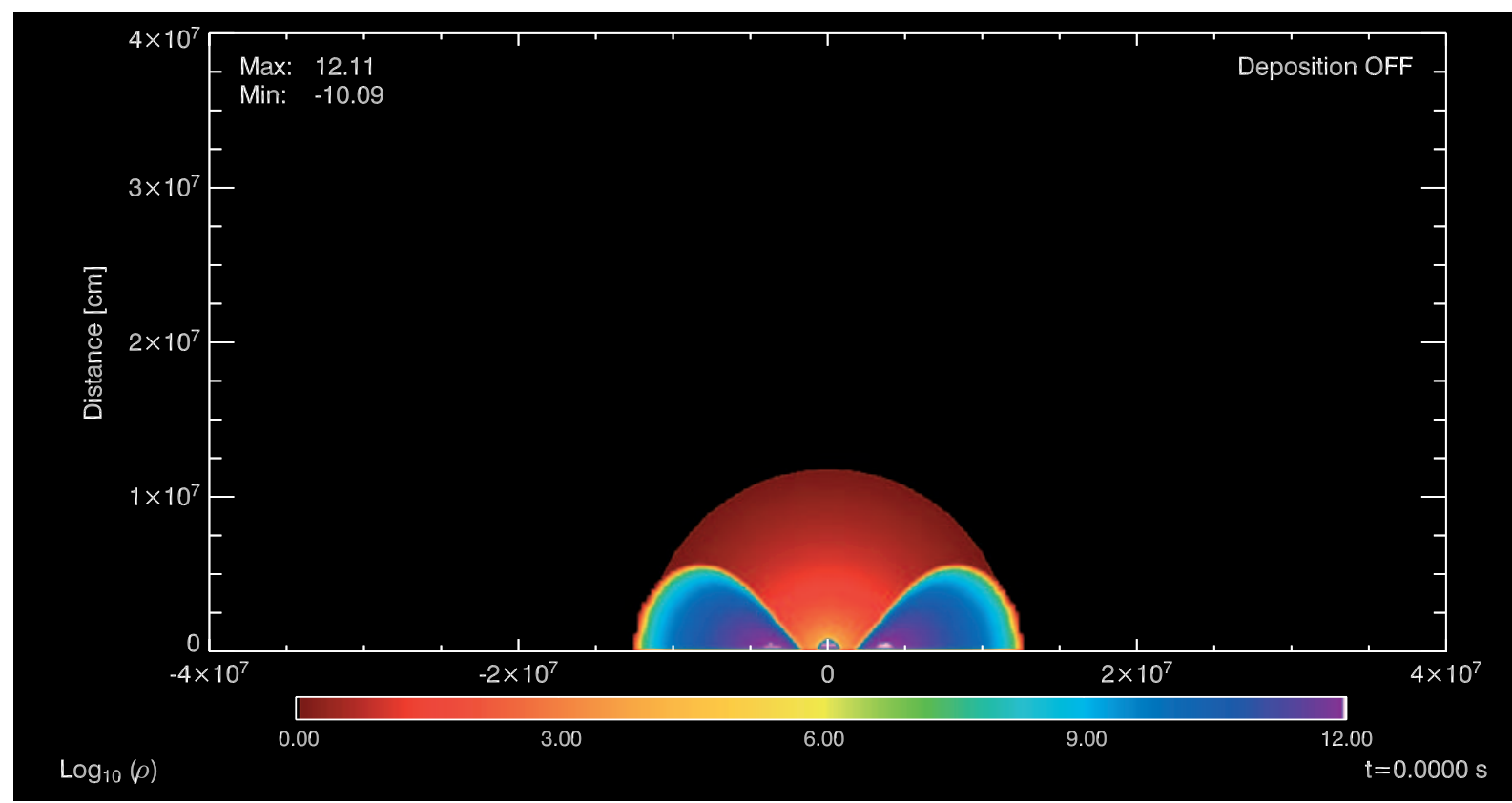

Fig. 2. Logarithm of the rest-mass density of the initial model of type-B simulations showing the structure of the accretion torus. The scale of the plot is limited in order to be able to distinguish properly the toroidal structure. In the upper left corner the maximum and minimum values of the displayed variable are reported. Note that in contrast to Fig. 1 the torus is much denser and more compact.

Witti et al. (1994). Complete ionization is assumed, and the effects due to possible degeneracy are neglected.

\section{Results}

Given the fact that our models do not include a fully consistent treatment of all relevant physics and our initial conditions are set up ad hoc instead of resulting from merger simulations of compact binary stars, we have performed a series of simulations trying to cover a physically meaningful parameter space. Nonetheless, we point out here that, even if we had done consistent simulations, the variety of resulting initial configurations could be very large because the properties of the forming black hole-accretion torus system and its environment are sensitive to a number of parameters which are incompletely known or variable (the masses and spins of the binary objects, the neutron star equation of state, general relativistic effects in the binary merging, etc.). 


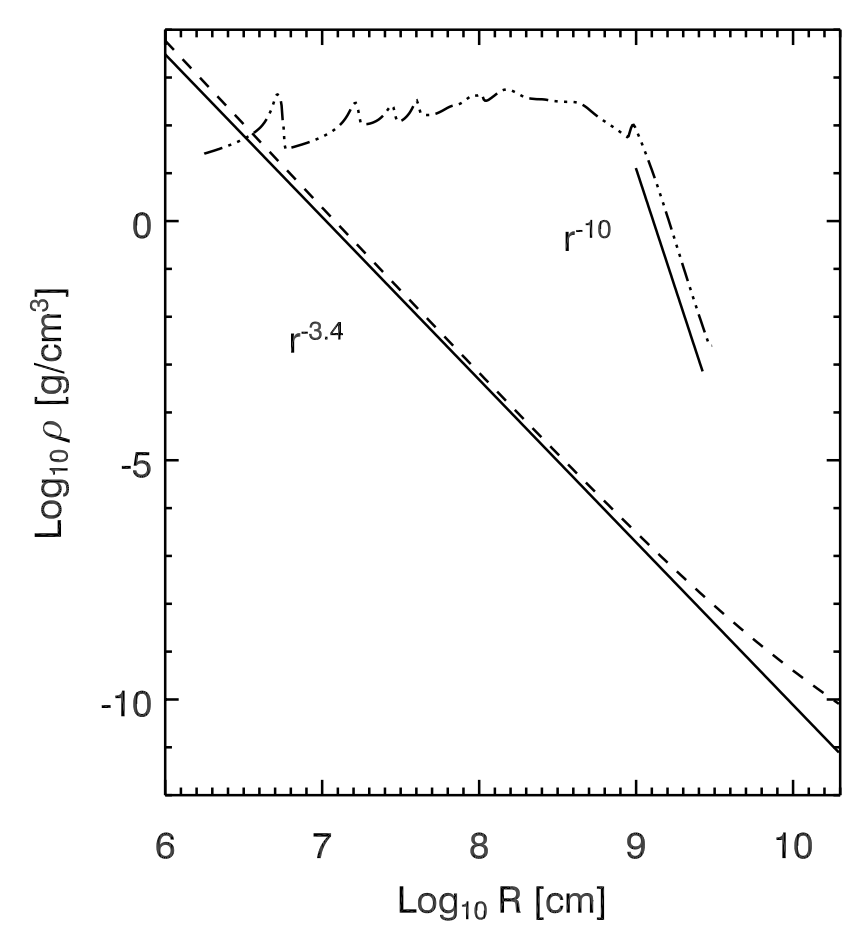

Fig. 3. Density vs radius along the symmetry axis of the two types of initial models. The dashed-dotted (dashed) line corresponds to initial models of type-A (type-B). The solid straight lines display power-law fits to both density distributions.

According to Ruffert \& Janka (1999), the accretion torus might be surrounded by a halo of relatively high density (a byproduct of the merger) that could extend up to a few hundred kilometers from the center of the merger. On the other hand, merger events should mostly occur in the interstellar medium and, therefore, a merger and its halo should be surrounded by a very low-density environment. The size and the exact density profile of the halo can strongly depend on, e.g., the physical parameters of the merging progenitors, the details of the merger dynamics, the time delay of the black hole formation, and the neutrino-driven baryonic outflow from a transient, massive, hot post-merging neutron star. A high density halo would have to be expected if, for example, the collapse to a black hole were delayed due to the effects of very rapid (differential) rotation or viscous heating (e.g., Duez et al. 2004; Morrison et al. 2004), in which case the hot neutron star would radiate neutrinos and a neutrino-driven wind (e.g., Duncan et al. 1986) would lead to a dense, expanding baryonic cloud around the merger site. It may also be possible to find situations where the accretion torus is surrounded by a thin, dilute halo, in particular, if the BH forms during the merger or within a few dynamical timescales afterwards (e.g., Shibata \& Uryū 2000; Shibata et al. 2003; Oechslin et al. 2004). In regard of these considerations we have performed two series of simulations. The first one (models A01 to A09 of Table 1) uses an initial model of type-A and corresponds to a case where the density of the halo is high and the density decline with distance is moderate (Fig. 3). The second set of models (B1 to B8) start from an initial model of type-B and, therefore, they correspond to the low-density halo class.

\subsection{The case of a high density halo}

For models of type-A, the parameter study comprises three different aspects. The first one is the study of the effects associated with a variation of the total energy deposition rate from $\dot{E}=10^{49} \mathrm{erg} \mathrm{s}^{-1}$ to $\dot{E}=5 \times 10^{51} \mathrm{erg} \mathrm{s}^{-1}$, fixing the value of the opening half-angle of the deposition region at $\theta_{0}=30^{\circ}$ and using a power law index of the energy deposition profile of $n=5$. The models computed for this purpose are A01, A02, A05 and A09 (Table 1). The second aspect is the dependence of the results on $\theta_{0}$ while keeping the rest of the parameters fixed ( $\dot{E}=2 \times 10^{50} \mathrm{erg} \mathrm{s}^{-1}, n=5$ ). The models used in this study are A02, A03 and A04 (Table 1). Finally, we have made two experiments where the energy released per unit of volume and time (the deposition rate density, hereafter) was kept constant but both $\dot{E}$ and $\theta_{0}$ were changed (models A03 and A08).

As a general conclusion, we find that, for energy deposition rates larger than a certain threshold, all the models lead to a relativistic or ultrarelativistic outflow (i.e., a fireball). This threshold is caused by the need to overcome the ram pressure $p_{\text {ram }}$ that the external medium exerts on the nascent fireball close to its initiation site. If the amount of energy per unit of time and per unit of volume that is pumped into the region extending up to the scale hight of the torus $\left(H \sim 10^{7} \mathrm{~cm}\right.$; note that about $97 \%$ of the energy is released in this region due to the large value of $n$ in Eq. (1)) in a time interval of about the freefall time of fluid elements at a distance of the order of $H$, is less than $p_{\text {ram }}$, then the fireball is unable to expand, and is swallowed by the $\mathrm{BH}$. The precise value of this threshold is model dependent. The threshold is higher for halos of higher density than for dilute ones. The shape of the fireball and its dynamical features depend very strongly on the type of initial model that is considered as will be discussed below.

\subsubsection{Dependence on the total energy deposition rate}

As we have already pointed out, there is a threshold energy deposition rate below which a relativistic fireball is not initiated. For models of type-A, the threshold is $\dot{E}_{\mathrm{thr}} \simeq 10^{49} \mathrm{erg} \mathrm{s}^{-1}$ (for $\theta_{0}=30^{\circ}$ ), while for models of type-B $\dot{E}_{\mathrm{thr}}<10^{48} \mathrm{erg} \mathrm{s}^{-1}$ (for $\theta_{0}=45^{\circ}$ ). The difference between both thresholds comes from the fact that the higher halo density of models of type-A leads to a higher ram pressure than in models of type-B.

In models of type-A, when $\dot{E}$ is above $\dot{E}_{\mathrm{thr}}$, the resulting outflow consists of either (i) a stratified (both in radial and polar directions) wind of approximately conical shape; or (ii) a relativistic jet. Let us note that, by definition, the difference between a wind and a jet is that in the latter the lateral boundaries are causally connected while they are not so in case of a wind. This distinction is relevant when the stability properties of both kind of solutions are considered ${ }^{1}$.

A wind results for high energy deposition rates per solid angle (models A05 and A09, Figs. 5, 6). In the polar direction, the wind is confined in a cone the angle of which is model dependent (see below). Its structure consists of a sheared wind with

\footnotetext{
${ }^{1}$ For example, a jet can be destroyed by Kelvin Helmholtz instabilities that originate at its surface, while a wind only develops a turbulent layer which does not affect the whole solution.
} 
Table 1. The first three columns show the model name and the two main parameters which characterize the energetics of the models, namely the energy deposition rate $\dot{E}$ (which represents the summed value for both hemispheres) and the half-opening angle of the cone over which the energy is released, respectively. For the models B07 and B08 entry $\dot{E}$ gives the peak values of the energy deposition rate $\left(\dot{E}_{0}\right.$, Eq. $\left.(10)\right)$. Columns four, five and six give some kinematic and dynamic properties of the outflow: the velocity $\left(\iota_{\mathrm{p}}\right)$ of the propagation of the fireball in the radial direction (i.e., the velocity of the leading, radial edge of the outflow), the maximum Lorentz factor $\left(\Gamma_{\max }\right)$ attained in the outflow and, the wind (or jet) half-opening angle $\left(\theta_{w}\right)$ of the outflow after $10 \mathrm{~ms}$ or $100 \mathrm{~ms}$, respectively. The last column gives the mass of the outflow $\left(M_{\mathrm{f}}\right)$ after $100 \mathrm{~ms}$. For models A04 and A06, there are data only up to $10 \mathrm{~ms}$. The half-opening angle of the outflow is computed as the maximum $\theta$-coordinate of all computational cells in where the fluid Lorentz factor exceeds a value of 10 and the radial velocity is positive. The mass of the fireball is computed by adding up the mass in all computational cells that match the same criterion as the one used to compute $\theta_{w}$. The choice of a relatively small value of the Lorentz factor $(\Gamma=10)$ in our criterion to separate the fireball matter from the external medium is motivated by the still ongoing acceleration of the fluid in the fireball that takes place at the expense of the large internal energy of the outflow.

\begin{tabular}{l|c|c|cc|cc|cc|c}
\hline \hline Model & $\dot{E}\left[\mathrm{erg} \mathrm{s}^{-1}\right]$ & $\theta_{0}$ & \multicolumn{2}{|c|}{$v_{\mathrm{p}}[c]$} & \multicolumn{2}{|c|}{$\Gamma_{\max }$} & \multicolumn{2}{|c|}{$\theta_{w}$} & $M_{\mathrm{f}}[\mathrm{g}]$ \\
& & & $(10 \mathrm{~ms})$ & $(100 \mathrm{~ms})$ & $(10 \mathrm{~ms})$ & $(100 \mathrm{~ms})$ & $(10 \mathrm{~ms})$ & $(100 \mathrm{~ms})$ & $(100 \mathrm{~ms})$ \\
\hline A01 & $10^{49}$ & $30^{\circ}$ & 0.67 & 0.62 & 18 & 18 & $<1^{\circ}$ & $<1^{\circ}$ & $4.0 \times 10^{15}$ \\
A02 & $2 \times 10^{50}$ & $30^{\circ}$ & 0.63 & 0.63 & 81 & 232 & $11.3^{\circ}$ & $6^{\circ}$ & $8.8 \times 10^{23}$ \\
A03 & $2 \times 10^{50}$ & $45^{\circ}$ & 0.80 & 0.67 & 11 & 27 & $9.5^{\circ}$ & $3.9^{\circ}$ & $4.5 \times 10^{24}$ \\
A04 & $2 \times 10^{50}$ & $75^{\circ}$ & 0.67 & - & 7 & - & $8.5^{\circ}$ & - & - \\
A05 & $10^{51}$ & $30^{\circ}$ & 0.99 & 0.82 & 84 & 562 & $15.0^{\circ}$ & $15^{\circ}$ & $3.5 \times 10^{25}$ \\
A06 & $10^{51}$ & $45^{\circ}$ & 0.97 & - & 80 & - & $15.8^{\circ}$ & - & - \\
A07 & $10^{51}$ & $75^{\circ}$ & 0.90 & 0.60 & 13 & 37 & $12.5^{\circ}$ & $8.13^{\circ}$ & $2.4 \times 10^{25}$ \\
A08 & $10^{50}$ & $31.4^{\circ}$ & 0.83 & 0.70 & 20 & 20 & $3.8^{\circ}$ & $2.9^{\circ}$ & $1.4 \times 10^{22}$ \\
A09 & $5 \times 10^{51}$ & $30^{\circ}$ & 0.70 & 0.97 & 91 & 748 & $23^{\circ}$ & $26^{\circ}$ & $3.3 \times 10^{26}$ \\
B01 & $2 \times 10^{50}$ & $45^{\circ}$ & 0.995 & 0.99994 & 33 & 247 & $36^{\circ}$ & $30^{\circ}$ & $5.4 \times 10^{24}$ \\
B02 & $2 \times 10^{50}$ & $60^{\circ}$ & 0.999 & 0.99995 & 40 & 274 & $35^{\circ}$ & $21^{\circ}$ & $5.0 \times 10^{24}$ \\
B03 & $2 \times 10^{50}$ & $75^{\circ}$ & 0.97 & 0.998 & 17 & 17 & $9.4^{\circ}$ & $2.3^{\circ}$ & $6.2 \times 10^{22}$ \\
B04 & $10^{49}$ & $45^{\circ}$ & 0.96 & 0.99991 & 30 & 244 & $30^{\circ}$ & $18^{\circ}$ & $3.2 \times 10^{23}$ \\
B05 & $10^{51}$ & $45^{\circ}$ & 0.999 & 0.99997 & 33 & 232 & $34^{\circ}$ & $28^{\circ}$ & $2.8 \times 10^{25}$ \\
B06 & $10^{50}$ & $41.4^{\circ}$ & 0.9991 & 0.99992 & 40 & 238 & $30^{\circ}$ & $23^{\circ}$ & $2.8 \times 10^{24}$ \\
B07 & $2.35 \times 10^{50}$ & $45^{\circ}$ & 0.995 & 0.99996 & 34 & 238 & $35^{\circ}$ & $28^{\circ}$ & $4.0 \times 10^{24}$ \\
B08 & $2.35 \times 10^{50}$ & $45^{\circ}$ & 0.999 & 0.99996 & 34 & 253 & $33^{\circ}$ & $24^{\circ}$ & $3.8 \times 10^{24}$ \\
\hline & & & & & & & & & \\
\end{tabular}

a central, low density, unshocked, ultrarelativistic part (near the symmetry axis) surrounded by a slow (mildly relativistic), denser, shocked layer. Surfaces of constant physical variables are almost spherical in the ultrarelativistic part of the wind. In the radial direction, the wind extends radially from a fiducial point (located at $\sim 3 \times 10^{6} \mathrm{~cm}$ ) to a terminal shock where it interacts with the external medium. Due to the larger density of the external medium, two shocks form: a reverse and a bow shock. Through the reverse shock the wind decelerates from ultrarelativistic velocities to mildly relativistic ones (between $0.6 c$ and $0.97 c$, see $v_{\mathrm{p}}$ in Table 1 ). The propagation speed of the bow shock is smaller than that of the fluid in the wind (Fig. 4). This explains why the radial expansion of the wind is only moderately relativistic.

In a conically expanding relativistic wind the physical variables change with the distance $z$ along the axis of rotational symmetry according to simple power laws (e.g., Levinson \& Eichler 2000). For example, the pressure, the density and the
Lorentz factor of a fluid whose adiabatic index is $\gamma=4 / 3$ follow the relations:

$p / p_{0}=\left(z / z_{0}\right)^{-4}, \rho / \rho_{0}=\left(z / z_{0}\right)^{-3}, \Gamma / \Gamma_{0}=z / z_{0}$,

where $p_{0}, \rho_{0}$ and $\Gamma_{0}$ are the values of the pressure, of the density and of the Lorentz factor at the fiducial point of the wind located at a distance $z_{0}$. In our models (Fig. 4), we obtain for the unshocked region of the outflow (the shocked one does not follow a power law)

$p / p_{0}=\left(z / z_{0}\right)^{-3.77}$
$\rho / \rho_{0}=\left(z / z_{0}\right)^{-2.98}$
$\Gamma / \Gamma_{0}=\left(z / z_{0}\right)^{0.925}$

The discrepancy in the power-law indices arises from the fact that the value of $\gamma$ is slightly smaller than $4 / 3$ in the wind of our models due to the presence of $\mathrm{e}^{+} \mathrm{e}^{-}$-pairs in the fluid. Since for type-A models the pressure and the density in the wind decrease faster than in the external medium up to $z \sim 10^{9} \mathrm{~cm}$ 


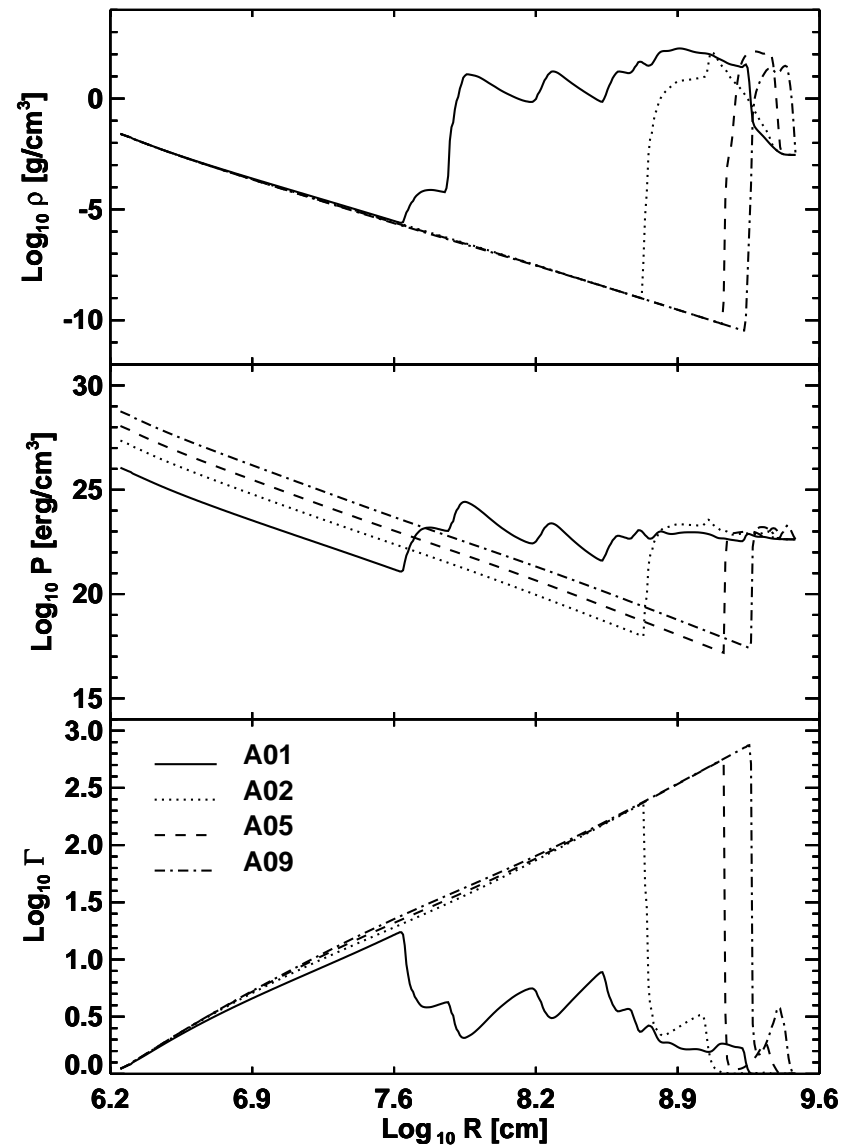

Fig. 4. Logarithm of the rest-mass density (top panel), of the pressure (middle panel) and of the fluid Lorentz factor (bottom panel) vs. radius along the symmetry axis for the models A01 (solid lines), A02 (dashed lines), A05 (dashed-dotted lines) and A09 (long-dashed lines) after $100 \mathrm{~ms}$. The displayed time is larger than the transition time in models A01 and A02 and, therefore, the recollimation of the initial ultrarelativistic wind solution yields relativistic jets in these two cases.

(Fig. 3) the outflow turns into a jet, because the wind pressure eventually becomes equal to that of the external medium. At that point, a recollimation shock forms starting from the wind surface and propagating towards the axis. Since the fiducial values of the pressure increase with an increasing $\dot{E}$, a higher $\dot{E}$ reduces the possibility of matching the pressure of the external medium. Therefore, the structure of the wind tends to remain conical when the energy deposition rate is increased. If the recollimation shock does not form before the fireball reaches the part of the environment where a steep density decline sets in $\left(\gtrsim 10^{9} \mathrm{~cm}\right.$; Fig. 3), it might not form at all (because for radial distances larger than $\sim 10^{9} \mathrm{~cm}$ the rate of decline of the pressure in the external gas exceeds that in the ultrarelativistic wind).

When the energy deposition rate is only slightly (model A01) or moderate (model A02) above the threshold, the conical wind converts to a relativistic jet due to the recollimation shock (Fig. 5). The outflow then possesses all the morphological elements known for light relativistic jets, i.e., a knotty, unsteady beam that ends in a Mach disc surrounded by an over-pressured cocoon. The outflow begins as a transient, expanding, over pressured, conical wind. But after a transition time $\left(\Delta t_{\text {trans }} \approx 10 \mathrm{~ms}\right.$ for model A01 and $\Delta t_{\text {trans }} \approx 30 \mathrm{~ms}$ for model A02) a recollimation shock forms due to the pressure confinement of the external medium reducing the opening angle to values less than $1^{\circ}$. The formation of the recollimation shock has two main consequences. First, it separates the outflow in two parts: an unshocked inner flow (close to the deposition region; the smooth region in Fig. 4), and an outher shocked flow where the pressure and the density have substantially larger values (Fig. 4). Second, the recollimation shock effectively stops the linear increase (Eq. (5)) of the beam Lorentz factor observed in the more energetic models (A05 and A09; see Fig. 4) even at large distances. Hence, the propagation speed of the head of the outflow is about $35 \%$ smaller in models A01 and A02 than that in models with higher energy deposition rates (Table 1) after the initial transient phase. As the fiducial pressure of the fireball grows with $\dot{E}$, while the pressure decrease with distance is approximately the same for the wind outflow in all models (Eq. (5)), the distance where the pressure of the wind and the external medium match increases with growing $\dot{E}$ (the same trend holds for $\Delta t_{\text {trans }}$ ). Whether pressure matching occurs or not depends on the value of the fiducial pressure and on the pressure gradient in the halo. Within $100 \mathrm{~ms}$ this happens in none of the more energetic models (A05 and A09).

Increasing the energy deposition rate in models of type-A leads to a progressive widening of the resulting fireball (Fig. 6). For models that produce an ultrarelativistic wind (but not a relativistic jet), Table 1 shows that the half-opening angle of the wind is smaller than the half-opening angle $\theta_{0}$ of the energy deposition cone (in case of a jet the half-opening angle is set by the interaction with the external medium and the cocoon dynamics). Thus, the opening angle is not determined by our ad-hoc choice of $\theta_{0}$. Instead, the opening angle is defined by the angular coordinate where the pressure of the fireball equals that of the torus. This angle depends on two factors: (i) the torus pressure at a given radial distance, increases as one moves from the axis to the equator; and (ii) the pressure of the fireball, at a given radial distance, is an increasing function of the energy deposition rate. Hence, as we increase $\dot{E}$ the location where the pressure of the fireball matches that of the torus shifts to larger angles, i.e., the opening angle of the outflow grows.

Within an outflow crossing time of the torus in the axial direction ( $\gtrsim 1 \mathrm{~ms}$ ), the fluid accelerates up to Lorentz factors of $\approx 10$ (Fig. 4) and, if it does not form a jet, the fluid becomes ultrarelativistic, i.e., any motion except in the radial direction is prevented. Hence, a sideways expansion is drastically reduced, and the initially imprinted opening angle remains unchanged. After $10 \mathrm{~ms}$ the maximum Lorentz factor rises to 100 in the case of winds while it is only $\sim 18$ for jets.

The amount of mass in the fireball (Table 1), defined as the sum of the masses of each computational cell in the outflow that moves out radially with a $\Gamma>10$, increases almost linearly with $\dot{E}$ during the period in which energy is released in the system. The mass is mainly concentrated in the cocoon that surrounds either the outflowing jet or wind. In the case of winds, only a small fraction of the mass moves at Lorentz factors larger than 100. 


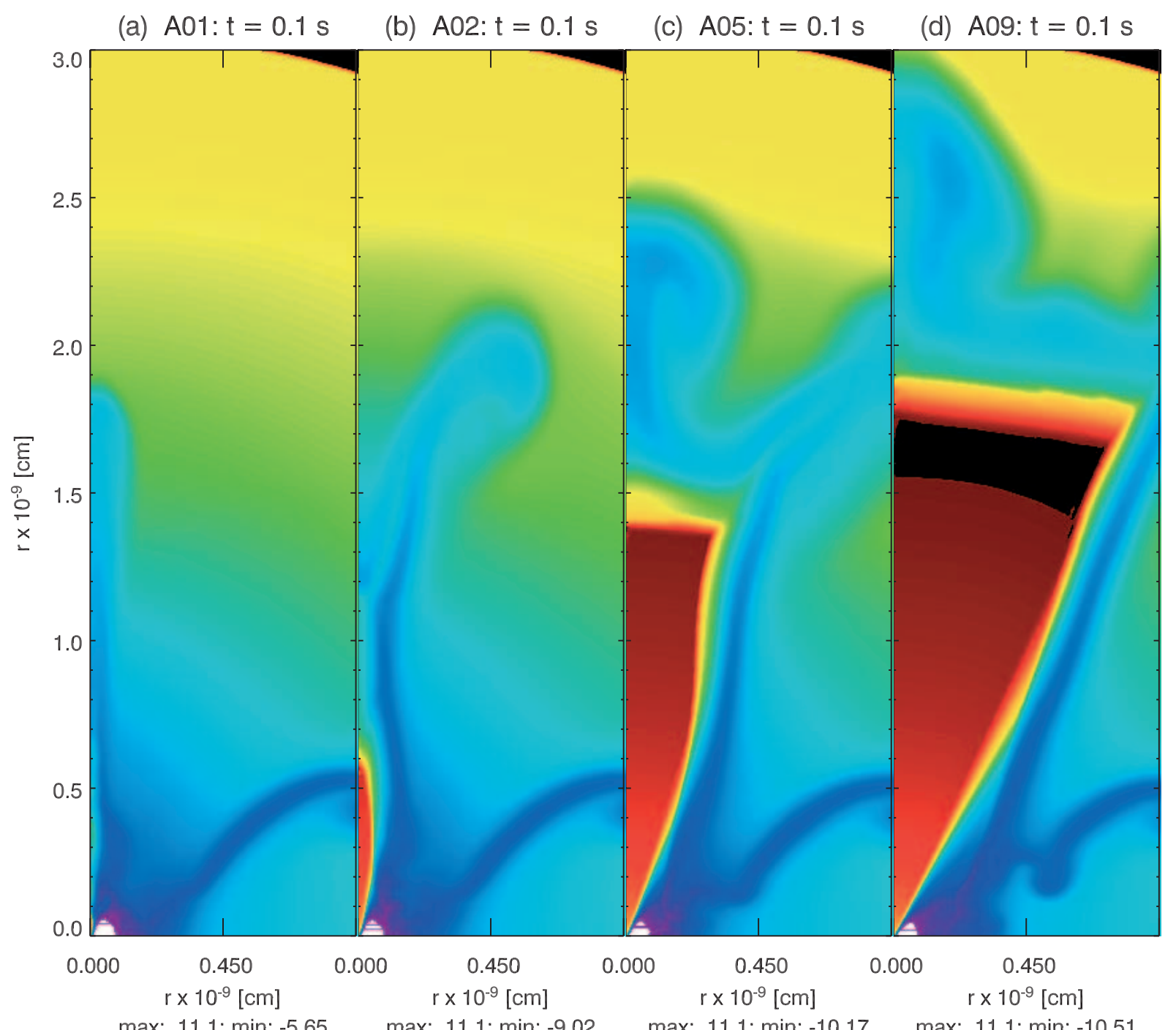

$\max : 11.1 ; \min :-5.65 \quad \max : 11.1 ; \min :-9.02 \quad \max : 11.1 ; \min :-10.17 \quad \max : 11.1 ; \min :-10.51$

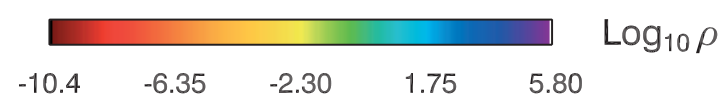

Fig. 5. Logarithm of the rest-mass density for models A01 (panel a)), A02 (panel b)), A05 (panel c)) and A09 (panel d)) after 100 ms of energy deposition. The color scale is limited in order to enhance the details of the outflow region. The maximum and minimum density values (in logarithmic scale) of each model are given below the panels.

\subsubsection{Dependence on the half-opening angle of the energy deposition cone}

We have performed a series of runs where we compared for fixed energy deposition rates the influence of an increasing angular width of the region of energy release, $\theta_{0}$ from $30^{\circ}$ to $75^{\circ}$. The models considered in this study are divided into two groups. The first group consists of A02, A03 and A04 (Table 1, Fig. 7) which have a moderate energy deposition rate of $\dot{E}=2 \times 10^{50} \mathrm{erg} / \mathrm{s}$ (group M hereafter). The second group possesses a higher deposition power of $10^{51} \mathrm{erg} / \mathrm{s}$, and consists of models A05, A06 and A07 (Table 1, Fig. 8; group H hereafter).

For a fixed, moderate energy deposition rate the opening angle of the outflow is initially quite insensitive to the increase of the deposition angle $\theta_{0}$ : for the M-group, while $\theta_{0}$ grows from $30^{\circ}$ to $75^{\circ}, \theta_{w}$ only decreases from $11.3^{\circ}$ to $8.5^{\circ}$ (we define $\theta_{w}$ as the angle of the outflow having $\Gamma>10$ ) in $10 \mathrm{~ms}$. After longer evolutionary times all the models of group $M$ tend to form relativistic jets with opening angles that depend on the interaction between the beam, the cocoon and the external medium, but not on $\theta_{0}$. For models of group $\mathrm{H}$, depending on the value of $\theta_{0}$, we obtain outflows with opening angles after $100 \mathrm{~ms}$ of evolution that exhibit a rather small range of variation between $15^{\circ}\left(\mathrm{A} 05 ; \theta_{0}=30^{\circ}\right)$ and $8.13^{\circ}\left(\mathrm{A} 07 ; \theta_{0}=75^{\circ}\right)$. Hence, the trend for group $\mathrm{H}$ is a slight non-monotonic decrease of the outflow opening angle as we deposit the energy in a wider region.

These results confirm, on the one hand, that the opening angle of the deposition region is not directly related to the 
(a) $\mathrm{A} 01: \mathrm{t}=0.1 \mathrm{~s}$

(b) $A 02: t=0.1 \mathrm{~s}$

(c) $\mathrm{A05}: \mathrm{t}=0.1 \mathrm{~s}$

(d) A09: $\mathrm{t}=0.1 \mathrm{~s}$

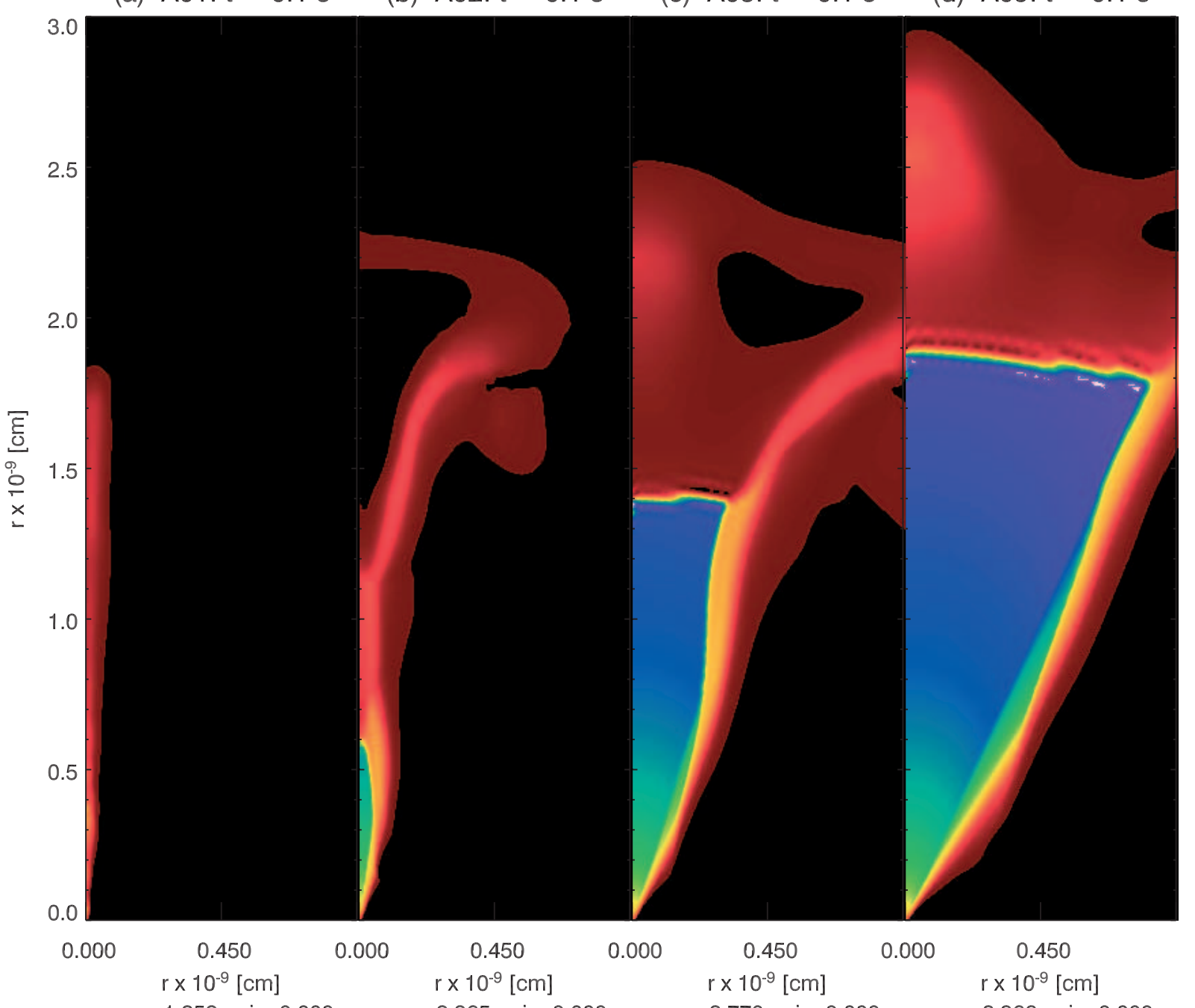

$\max : 1.252$; $\min : 0.000 \quad \max : 2.365 ; \min : 0.000 \quad \max : 2.770 ; \min : 0.000$ max: 2.966 ; $\min : 0.000$

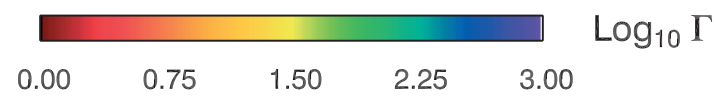

Fig. 6. Logarithm of the Lorentz factor for models A01 (panel a)), A02 (panel b)), A05 (panel c)) and A09 (panel d)) after 100 ms of energy deposition. The color scale is limited in order to enhance the details of the outflow region. The maximum and minimum density values (in logarithmic scale) for each model are given below the panels.

final opening angle of the outflow and, on the other hand, that the mechanism of collimation of this series of models is significantly different from the mechanism discussed by Levinson \& Eichler (2000). These authors propose that a baryon-rich wind emerging from the torus is able to collimate the baryon poor jet moving along the symmetry axis of the system. For type-A models the role of such a baryon-rich wind is played by an almost static cloud that is formed during the initial relaxation phase of the torus. This cloud has a rather low velocity $(\simeq 0.027 c)$ and a density of $\simeq 10^{6} \mathrm{~g} / \mathrm{cm}^{-3}$ at a distance of $10^{7} \mathrm{~cm}$. The density does not fall off as $r^{-2}$ (as in case of the baryon-rich wind of Levinson \& Eichler 2000), but with a much smaller power. Therefore, the main effect of the environment is, in our case, to provide sufficient inertial confinement for preventing excessive sideways expansion of the axial ultrarelativistic flow.
The insensitivity of $\theta_{w}$ to $\theta_{0}$, arises because the opening angle of the relativistic outflow is mainly determined by the inclination angle of the torus walls around the rotation axis. As discussed in Sect. 3.1.1, the torus does not have a sharp surface that separates it from the halo. Instead, a gradient of density and pressure connects it with the surrounding medium. We have argued that the region where the pressure of the torus and of the emerging fireball match each other determines the initial $\theta_{w}$. In the considered set of models, the energy deposition rate per unit of volume decreases with growing $\theta_{0}$. This fact implies a smaller pressure (at any given radial distance). Thus, pressure equilibrium between the fireball and the torus occurs at a smaller polar angle, which in turn reduces the value of $\theta_{w}$ as can be seen in Figs. 7 and 8 . 
(a) A02: $t=0.01 \mathrm{~s}$

(b) A03: $\mathrm{t}=0.01 \mathrm{~s}$

(c) $\mathrm{A} 04: \mathrm{t}=0.01 \mathrm{~s}$
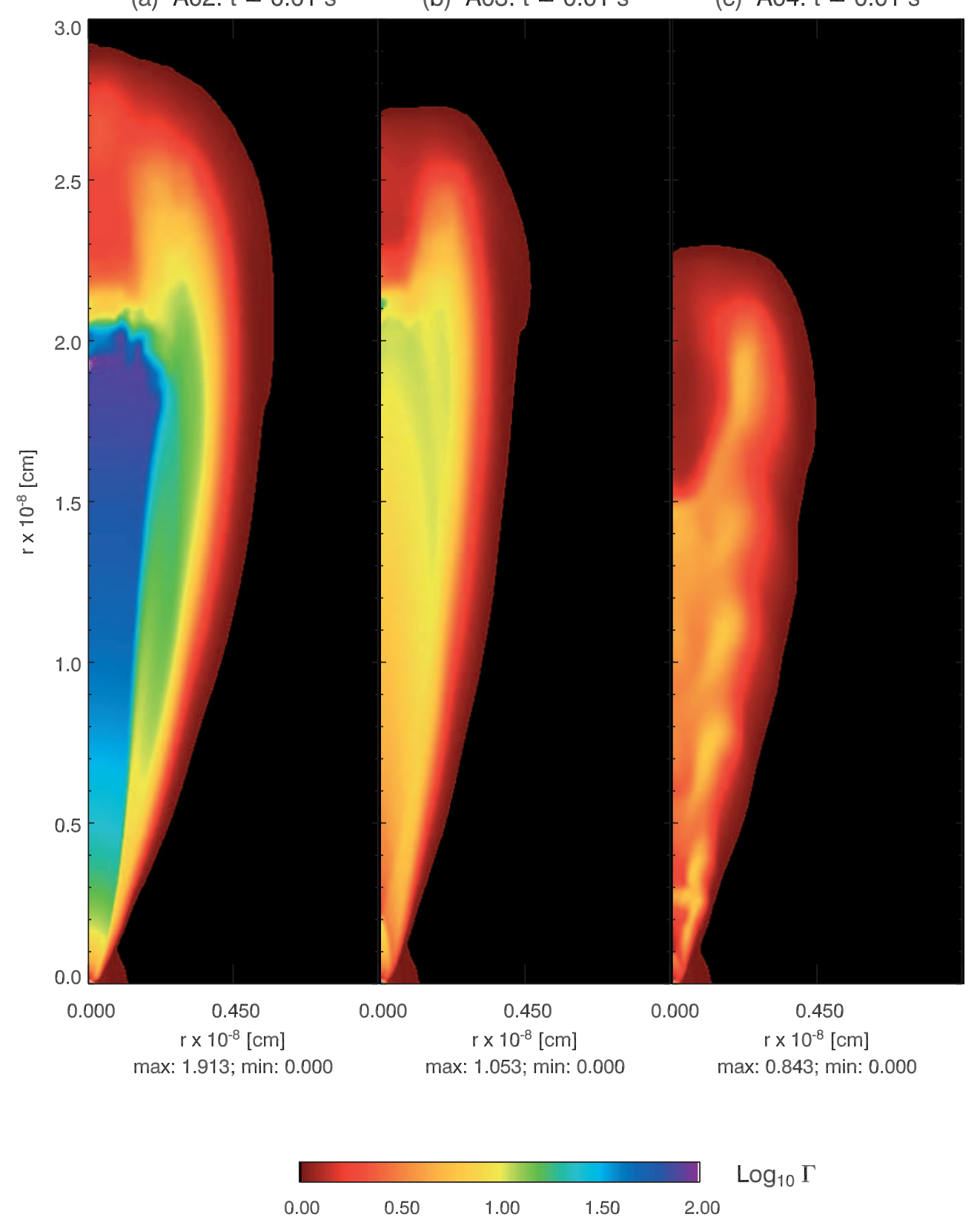

Fig. 7. Logarithm of the Lorentz factor for models A02 (panel a)), A03 (panel b)) and A04 (panel c)) after 10 ms of energy deposition. The color scale is limited in order to enhance the details of the outflow region. The maximum and minimum Lorentz factor values (in logarithmic scale) of each model are given below the panels.

Another consequence of increasing $\theta_{0}$ is the transition from a sheared, ultrarelativistic wind to a knotty, moderately relativistic jet. For models of group $\mathrm{M}$ and $\theta_{0} \geq 45^{\circ}$, this transition takes place very soon after the birth of the fireball ( $\Delta t_{\text {trans }} \lesssim$ $10 \mathrm{~ms}$; Fig. 7) at small radial distances (models A03 and A04). In the case of the largest $\theta_{0}$ considered (model A04, $\theta_{0}=75^{\circ}$ ), the maximum Lorentz factor is only 6.97 , and the jet is prone to large amplitude Kelvin-Helmholtz (KH) instabilities that lead to a wrinkled surface and an inhomogeneous beam. Increasing the energy deposition rate (models of group $\mathrm{H}$ ), we observe the same qualitative behavior, but the wind-to-jet transition happens at a larger value of $\theta_{0}\left(\approx 75^{\circ}\right)$.
Model A04 produces an inhomogeneous, low-velocity jet because the imposed energy deposition rate per unit volume for the chosen value of $\theta_{0}$ is only slightly above the threshold value of $\dot{E}$ that is necessary to launch an outflow at all. We have confirmed this by performing another simulation with the same energy deposition rate but with $\theta_{0}=90^{\circ}$, which did not produce any outflow. For a given threshold value for the energy deposition rate per unit of volume, the minimum total energy deposition rate to produce an outflow increases with larger $\theta_{0}$. The formation of jets or winds depends on the energy released per unit of time and of volume. The larger the energy release, the larger is the chance for wind formation. 

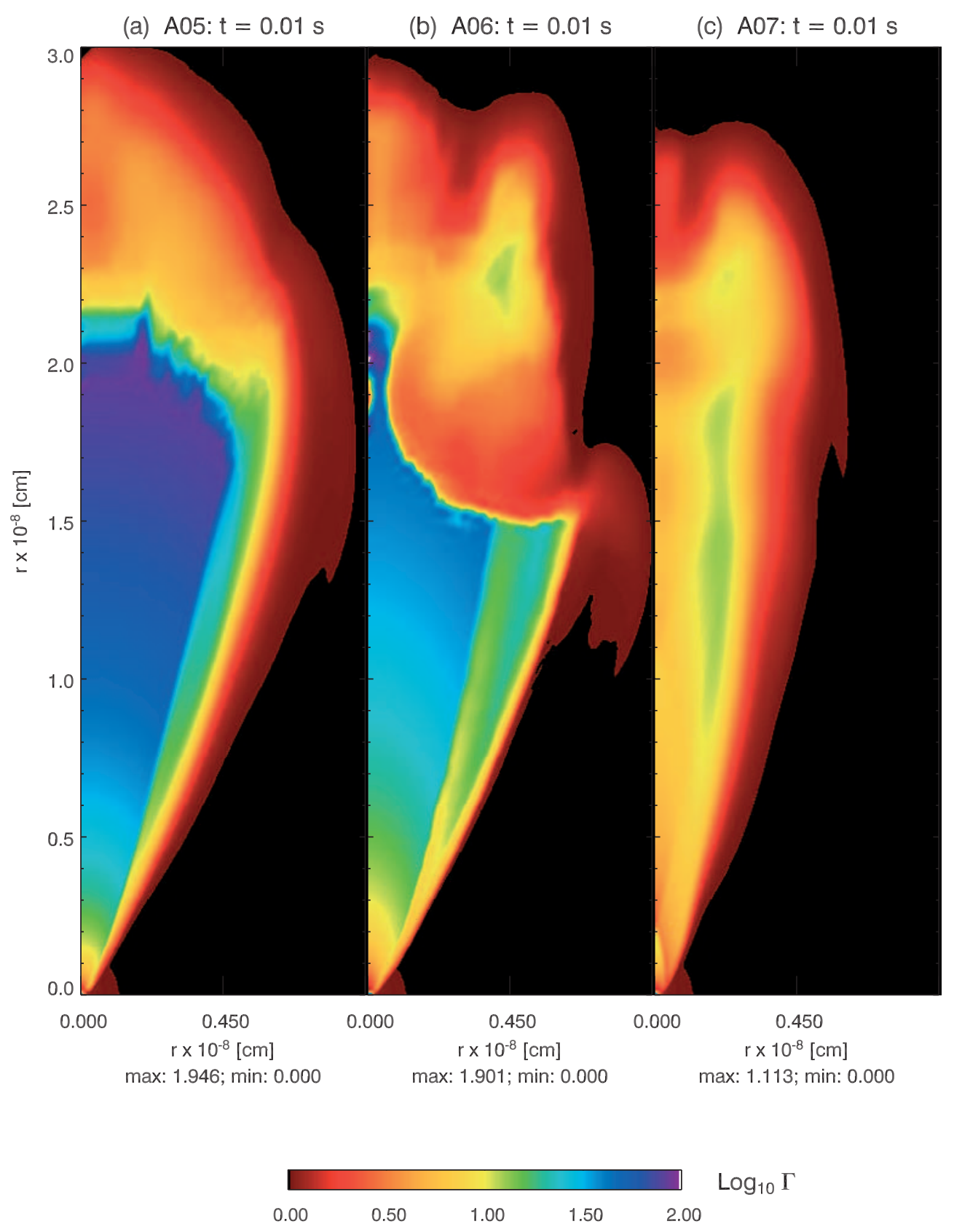

Fig. 8. Logarithm of the Lorentz factor for models A05 (panel a)), A06 (panel b)) and A07 (panel c)) after 10 ms of energy deposition. The color scale is limited in order to enhance the details of the outflow region. The maximum and minimum Lorentz factor values (in logarithmic scale) of each model are given below the panels.

\subsubsection{Dependence on the deposition volume $V_{\text {dep }}$ and on the total energy deposition rate $\dot{E}$ for $\dot{E} / V_{\text {dep }}=$ constant}

In this section we will study the dependence on the deposition volume if the rate per unit volume at which energy is released in the system is fixed. The two models that we consider here are A03 (having a large deposition rate and a large deposition angle) and A08 (with smaller deposition rate and deposition angle but the same energy released per unit of time and volume as A03). Both models develop a mildly relativistic jet and display very similar properties at the beginning of the evolution (Fig. 9). However, their later evolution differs.
For example, the propagation velocity of the jet head is smaller for A03 than for A08, because model A03 accumulates more mass in the cocoon, and a part of this mass enters the beam thus leading to a more massive jet $\left(M_{\mathrm{f}}^{A 03}=4.5 \times 10^{24} \mathrm{~g}\right.$ while $M_{\mathrm{f}}^{A 08}=1.4 \times 10^{22} \mathrm{~g}$ ).

This can be understood from the fact that in model A03 the cone where energy is deposited has a larger half-opening angle $\theta_{0}$ and thus overlaps more with the outer layers of the torus than it does in model A08. The energy release in this overlap region causes mass shedding from the outer layers of the torus where the densities (for $31.4^{\circ}<\theta<45^{\circ}$ ) are 2 to 4 orders of magnitude higher than those in the solid angle corresponding to the deposition cone of model A08. Hence, the outflow in 

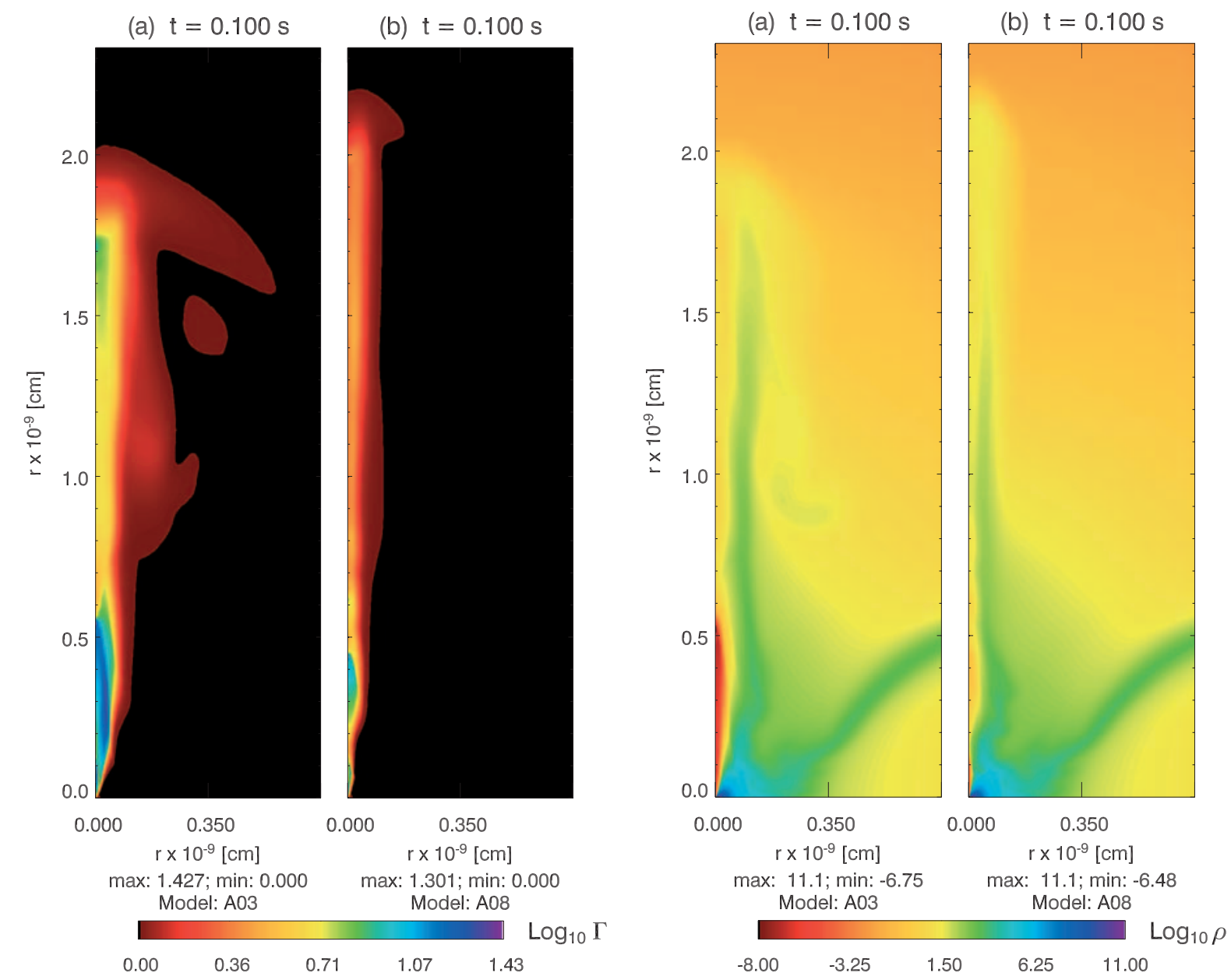

Fig. 9. Logarithm of the rest mass density (right two panels) and of the Lorentz factor (left two panels) for models A03 (panels a)), and A08 (panels b)) after $100 \mathrm{~ms}$ of energy deposition. The color scales are limited in order to enhance the details of the outflow region. The maximum and minimum values of the respective variable are given below the panels. Note the strong pinching of the jet of model A08 at $r \approx 10^{8} \mathrm{~cm}$ which is due to the shedding of the most external shells of the accretion torus.

model A03 becomes baryon-enriched. The outflow velocities are correspondingly lower, although the rate of energy deposition per unit volume is the same as in model A08.

Independent of the differences in mass, velocity and maximum Lorentz factors reached in the two models, the final opening angle becomes less than $4^{\circ}$ in both cases (Table 1), because a series of recollimation shocks in the beam prevents a large sideways expansion of the forming jet. Nonetheless, the cocoon of the jet of model A03 is twice as thick $\left(\approx 3 \times 10^{8} \mathrm{~cm}\right.$ when the jet has propagated for $100 \mathrm{~ms}$ ) as that of model A08. These differences in the cocoon can be understood from the different angular extensions of the deposition regions, too.

\subsubsection{Evolution after the switch off of the central energy source}

We have done simulations where, starting from model A09 (which has the largest $\dot{E}$ of all type-A models), the energy deposition was switched off after a source activity time $t_{\mathrm{sa}}=$ $100 \mathrm{~ms}$. In this case the ultrarelativistic wind solution was destroyed within less than $2 t_{\mathrm{sa}}$, because the fireball sweeps up mass when it moves through the high density halo in models of
type-A (Figs. 10, 11). The swept up mass prevents the terminal shock of the ultrarelativistic wind from moving at ultrarelativistic speeds (it actually propagates at a speed of $\approx 0.97 c$ at $100 \mathrm{~ms}$ ). Therefore the ultrarelativistic wind is decelerated when it catches up with the reverse shock (Figs. 10b, 11b). In case of type-A models that form jets, a similar argument can be used to explain the mildly relativistic speeds at which the heads of the jets propagate $(\approx 0.67 c)$. Hence, models of type-A will, in general, not lead to successful GRBs, because they accumulate too much mass in the fireball and decelerate well before the outflow becomes optically thin.

Note that a necessary condition for a fireball to produce a successful GRB is that the rest mass carried by the fireball multiplied by $c^{2}$ should be about two orders of magnitude smaller than the energy of the fireball by the time at which transparency sets in. If the mass of the fireball grows too much before the fireball reaches the photosphere, the Lorentz factor corresponding to the terminal shock of the wind, and as a consequence, the fluid Lorentz factor, will be reduced below the critical limit (namely, $\Gamma \approx 100$ ) to account for the properties of observed GRBs. An estimate of the maximum Lorentz factor the fireball could ultimately reach (if all the internal 

(a) A09: $\mathrm{t}=0.130 \mathrm{~s}$
(b) $A 09: t=0.200 \mathrm{~s}$
(c) A09: $\mathrm{t}=0.383 \mathrm{~s}$

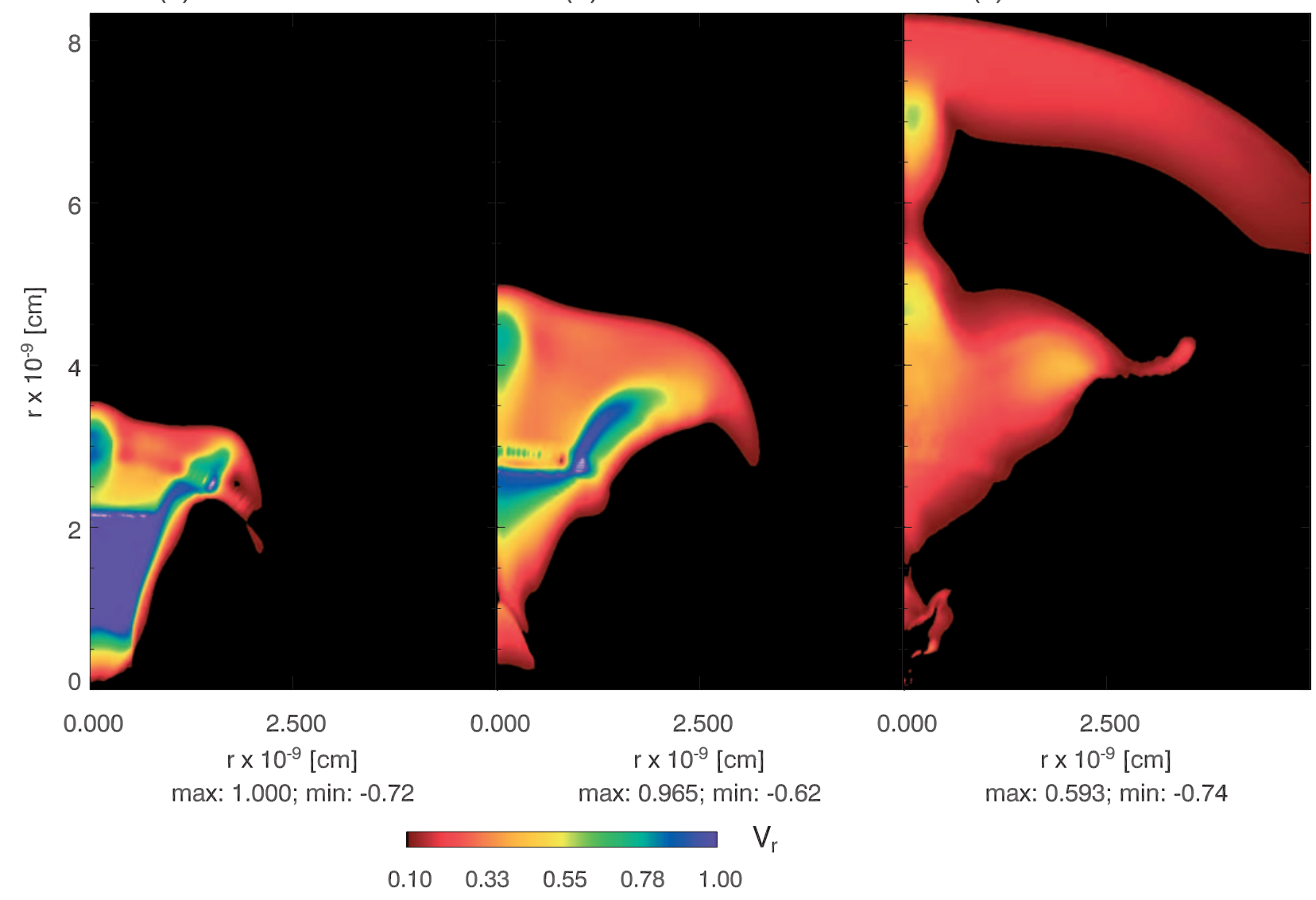

Fig. 10. Snapshots of the radial velocity of model A09 after the shutdown of the energy release (the snapshot times are provided above each panel). The color scale is limited in order to enhance the details of the outflow region. The maximum and minimum radial velocity values of each model are given below the panels. Although the outflow has reached Lorentz factors above 700 after 100 ms of evolution, and continues accelerating (panel a)) until the rear end of the outflow catches up with the reverse shock (panel b)), after 500 ms only a subrelativistic outflow has survived and propagates with radial velocities of $\sim 1.25 \times 10^{10} \mathrm{~cm} \mathrm{~s}^{-1}$.

energy could be converted into kinetic energy) is given by the ratio $\eta \equiv E / M c^{2}$, where $E$ is the total energy of the fireball at any given time after the energy deposition is switched off. $M$ is the mass of the fireball computed according to the definition given in the caption of Table 1. In model A09 $\eta$ decreases with time once the energy deposition is switched off, because of the increasing mass of the fireball. Thus, this model will not meet the criterion $\eta \approx 100$ which is required for producing an observable GRB.

Although type-A models are not expected to produce GRBs, we can estimate the kind of observational signature that this kind of events might generate. We assume that after some hundred milliseconds of evolution the bipolar outflow can be roughly approximated by two spherical, homogeneous clouds of hot radiation-dominated plasma, each with a mass $M \sim 10^{-5} M_{\odot}$, radius $R_{0} \sim 10^{9} \mathrm{~cm}$, temperature $T_{0} \sim 1.5 \times 10^{9} \mathrm{~K}$ and internal energy $U_{0} \sim 2 \times 10^{50} \mathrm{erg}$ (Figs. 10c, 11c). Assuming that the subsequent evolution of such a cloud is adiabatic, its temperature and internal energy will decrease as the cloud expands. Transparency is reached when the radius of the plasma cloud becomes

$R_{\mathrm{t}} \approx 3 \times 10^{13} \mathrm{~cm}\left(\frac{\kappa}{\kappa_{\mathrm{e}}}\right)^{1 / 2}\left(\frac{M}{10^{-5} M_{\odot}}\right)^{1 / 2}$ on a time scale (assuming that the cloud expands with about the light speed)

$t_{\mathrm{t}} \approx R_{\mathrm{t}} / c \approx 10^{3} \mathrm{~s}\left(\frac{\kappa}{\kappa_{\mathrm{e}}}\right)^{1 / 2}\left(\frac{M}{10^{-5} M_{\odot}}\right)^{1 / 2}$,

where $\kappa \approx \kappa_{\mathrm{e}} \approx 0.2 \mathrm{~cm}^{2} \mathrm{~g}^{-1}$ is the mean opacity (with $\kappa_{\mathrm{e}}$ being the opacity caused by electron scattering for an electron-tobaryon ratio of 0.5 ).

When transparency sets in, both the temperature and the internal energy of the cloud have decreased to the following values

$$
\begin{aligned}
T_{\mathrm{t}} \approx & 5 \times 10^{4} \mathrm{~K}\left(\frac{T_{0}}{1.5 \times 10^{9} \mathrm{~K}}\right)\left(\frac{\kappa}{\kappa_{\mathrm{e}}}\right)^{-1 / 2} \\
& \times\left(\frac{M}{10^{-5} M_{\odot}}\right)^{-1 / 2}\left(\frac{R_{0}}{10^{9} \mathrm{~cm}}\right), \\
U_{\mathrm{t}} \approx & 7 \times 10^{45} \mathrm{erg} \mathrm{cm}^{-3}\left(\frac{T_{0}}{1.5 \times 10^{9} \mathrm{~K}}\right)^{4}\left(\frac{\kappa}{\kappa_{\mathrm{e}}}\right)^{-1 / 2} \\
& \times\left(\frac{M}{10^{-5} M_{\odot}}\right)^{-1 / 2}\left(\frac{R_{0}}{10^{9} \mathrm{~cm}}\right)^{4} .
\end{aligned}
$$




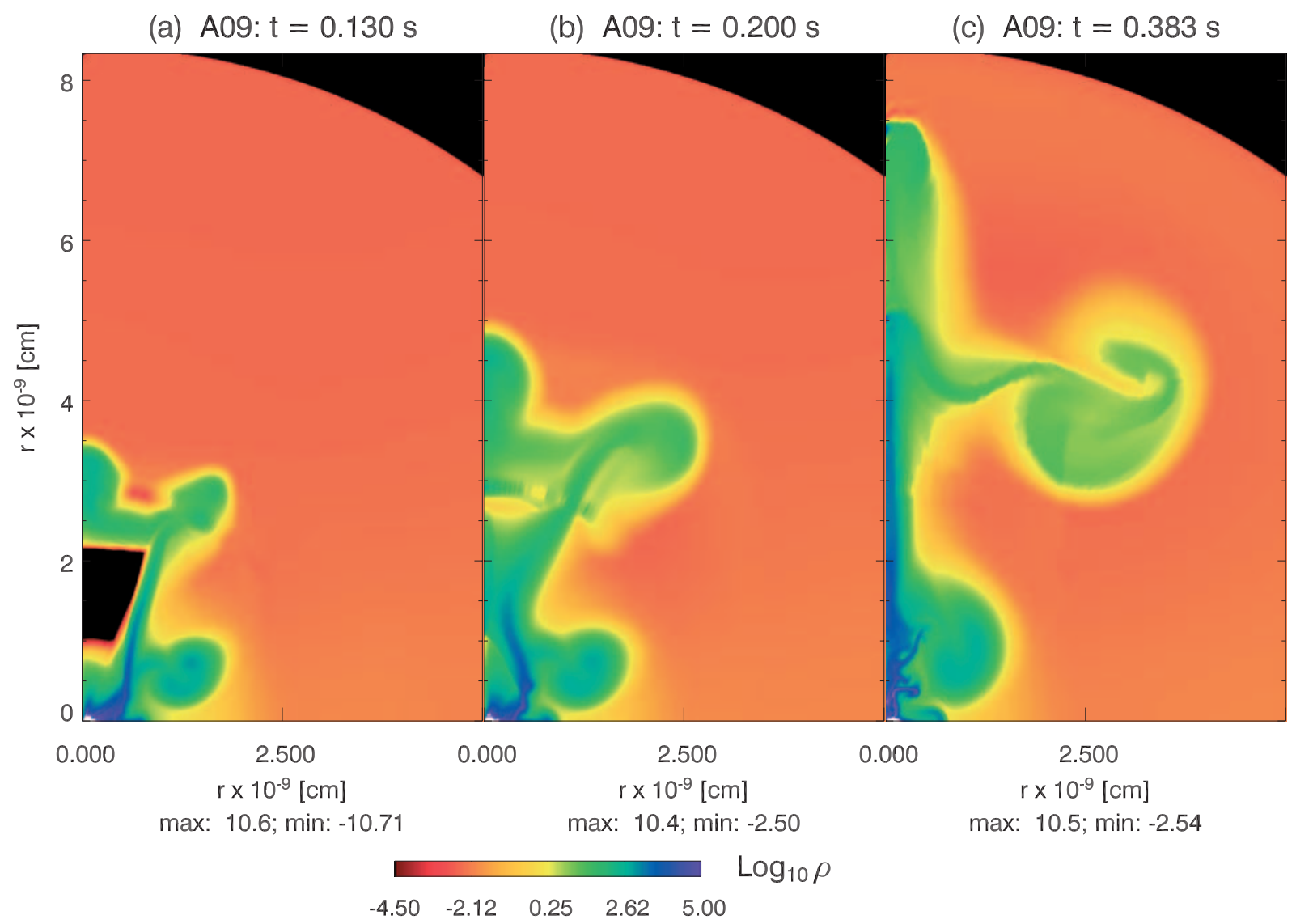

Fig. 11. Snapshots of the logarithm of the rest mass density of model A09 before the shutdown of the energy release (the snapshot times are provided above each panel). The color scale is limited in order to enhance the details of the outflow region. The maximum and minimum rest mass density values (in logarithmic scale) of each model are given below the panels.

After optically thin conditions are reached, most of the internal energy of the cloud will be radiated over a time scale of the order of $t_{\mathrm{t}}$ and, therefore, the peak luminosity $L_{\mathrm{m}} \approx U_{\mathrm{t}} / t_{\mathrm{t}}$ will be roughly

$$
\begin{aligned}
L_{\mathrm{m}} \approx & 7 \times 10^{42} \mathrm{erg} \mathrm{s}^{-1}\left(\frac{T_{0}}{1.5 \times 10^{9} \mathrm{~K}}\right)^{4}\left(\frac{\kappa}{\kappa_{\mathrm{e}}}\right)^{-1} \\
& \times\left(\frac{M}{10^{-5} M_{\odot}}\right)^{-1}\left(\frac{R_{0}}{10^{9} \mathrm{~cm}}\right)^{4} .
\end{aligned}
$$

Therefore, we expect a low-luminosity, soft UV-flash to be emitted as a result of the nonrelativistic outflow expanding from $\mathrm{BH}$-tori systems in high-density merger halos. Due to the small value of $L_{\mathrm{m}}$ only nearby events might be detectable.

\subsection{The case of low-density halo}

In case of a type-B progenitor, our parameter study comprises the same three different aspects as for type-A models. We first study the dependence on the energy deposition rate which is varied from $10^{49} \mathrm{erg} \mathrm{s}^{-1}$, to $2 \times 10^{50} \mathrm{erg} \mathrm{s}^{-1}$ keeping the value of the half-opening angle of the deposition cone fixed to $\theta_{0}=45^{\circ}$ and the power-law index of the vertical decrease of the energy deposition rate set to $n=5$ (see Sect. 3.1). The corresponding models are B01, B04 and B05 (Table 1). The second aspect is the dependence of the results on $\theta_{0}$ for $\dot{E}=2 \times 10^{50} \mathrm{erg} \mathrm{s}^{-1}$ and $n=5$ (models B01, B02 and B03; Table 1). Thirdly we compare models where the energy deposition rate per unit volume is fixed, but $\theta_{0}$ was varied (models B02 and B06).

We have further computed two models where the energy deposition rate was assumed to vary with time (models B07 and B08). These models were set up according to the following prescription for the energy deposition rate (guided by the results obtained by Setiawan et al. 2004)

$\dot{E}_{\mathrm{B} 07}= \begin{cases}\dot{E}_{0} \frac{t}{0.01} & \text { for } t \leq 0.01 \mathrm{~s}, \\ \dot{E}_{0} & \text { for } 0.01 \mathrm{~s}<t \leq 0.03 \mathrm{~s}, \\ \dot{E}_{0}\left(\frac{t}{0.03}\right)^{-3 / 2} & \text { for } t>0.03 \mathrm{~s},\end{cases}$

where $\dot{E}_{0}=2.35 \times 10^{50} \mathrm{erg} \mathrm{s}^{-1}$. With this value of $\dot{E}_{0}$ the same amount of energy is released in model B07 until time infinity as in model B01 during its on-time of $100 \mathrm{~ms}$. We point out that due to the rather steep power-law decrease of the energy deposition rate with time in B07, more than $60 \%$ of the total energy is released within the first $100 \mathrm{~ms}$. In model B08, the energy deposition rate follows the same average time dependence as in B07 but is sinusoidally modulated with a time period $t_{\mathrm{var}}=10 \mathrm{~ms}$ according to

$\dot{E}_{\mathrm{B} 08}=\dot{E}_{\mathrm{B} 07} \cdot\left(0.5 \sin \left(2 \pi t / t_{\mathrm{var}}\right)+1\right)$.

In all other models of type-B we have initiated the energy deposition by raising the energy deposition rate linearly from zero 
to its terminal value within $10 \mathrm{~ms}$. This timescale leads to a more modest onset of the hydrodynamic evolution. It can be motivated by the fact that in neutron star mergers the neutrino luminosities and, therefore, the energy deposition by $v \bar{v}$ annihilation increase within a few milliseconds in response to the viscous shear heating of the merger remnant (see, e.g., Janka et al. 1999; Rosswog \& Liebendörfer 2003).

In type-A models, a relativistic or ultrarelativistic outflow (i.e., a fireball) is obtained when the energy deposition rate is larger than a certain threshold value (Sect. 3.1). The threshold for type-B models is much smaller than that for models of type- $\mathrm{A}$ in case the opening angle of the deposition cone is chosen to be the same (for $\theta_{0}=30^{\circ}$ the threshold is well below $10^{48} \mathrm{erg} \mathrm{s}^{-1}$ ). This can be understood by the lower ram pressure that the low-density gas exerts on the fireball close to its site of initiation in type-B models.

The shape and the internal structure of the fireballs in models of type-B (e.g., Figs. 15 and 16) differ substantially from those of type-A models (e.g., Fig. 6). Fireballs in type-B models that reach ultrarelativistic speeds (all the B-cases shown in Table 1 except B03) display a conical core with an opening angle of $\approx 5^{\circ}$ to $\approx 20^{\circ}$ which spreads into a $26^{\circ}$ to $35^{\circ}$ wide conical region at larger radii. In addition, the full structure is surrounded by an approximately spherical shell of matter that expands with moderate Lorentz factors $(<10)$. In this shell the variation of the Lorentz factor with polar angle depends strongly on the model. This structure is produced by the deposition of energy in the surface layers of the torus. Different from models of type-A, the unshocked region of the outflow (i.e., the region of the outflow between the fiducial point and the first recollimation shock) extends to small radial distances (after $100 \mathrm{~ms}$, this region covers $\lesssim 10^{7} \mathrm{~cm}$; Fig. 12, except for models B02 and B03) and there is no simple stratification in the angular direction. In models of type- $\mathrm{B} \mathrm{KH}$ instabilities originating in the walls of the torus are stronger. They produce variations of Lorentz factor (Fig. 13) and density (Fig. 14) in the outflow, in particular close to the angular edge of the central conical structure. In the radial direction the KH instabilities cause a modulation of the on-average increasing Lorentz factor, and local fluctuations of the density and the pressure.

The growth of $\mathrm{KH}$ modes from the interface between two relativistic fluids depends, among other parameters, on the contrast of density $(\chi)$ and of specific enthalpy between the two fluids. Roughly, the larger the density contrast is, the faster the growth of the $\mathrm{KH}$ modes. Thus, the faster growth of $\mathrm{KH}$ instabilities close to the accretion torus in most of the models of type-B can be attributed to the larger density contrast between the torus and the outflow $\left(\chi \equiv \rho_{\text {torus }} / \rho_{\text {outflow }} \simeq 10^{14}\right.$ ) as compared to models of type-A $\left(\chi \simeq 10^{12}\right)$. As a consequence of the growth of $\mathrm{KH}$ instabilities, mass is entrained in the relativistic outflow which modifies the local speed of the fluid. The total mass entrained is comparable to that found for typeA models. However, in contrast to those models, the amount of matter piled up in the front part of the outflow is much smaller now (because the halo is less dense). This allows a highly relativistic propagation velocity of the fireballs of type-B models (Table 1). Indeed, the propagation speed is so large that

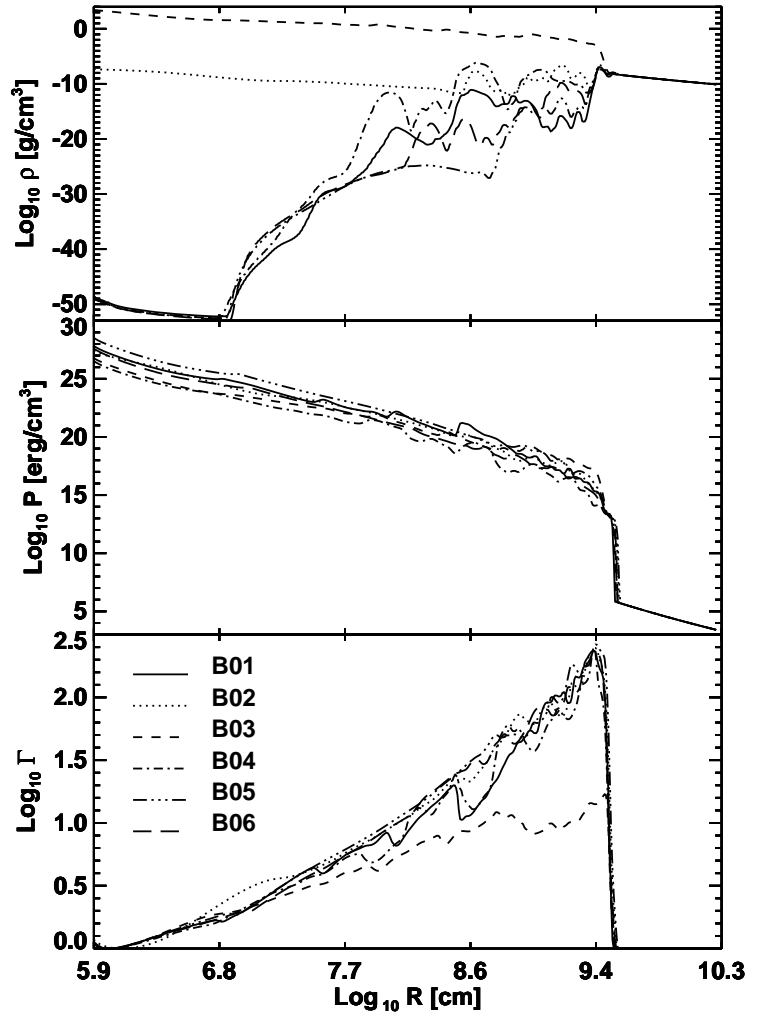

Fig. 12. Logarithm of the rest-mass density (top panel; in $\mathrm{g} \mathrm{cm}^{-3}$ ), of the pressure (middle panel) and of the fluid Lorentz factor (bottom panel) vs. radius along the symmetry axis for the models B01 to B06 after $100 \mathrm{~ms}$.

models of type-B are viable to produce GRBs as will be further discussed in Sect. 4.

The mixing of non-relativistic baryons with the relativistic fluid of the outflow leads to a change of the adiabatic index in the fireball which occurs mainly along the boundaries of the outflow. In a cone of about $20^{\circ}$ the outflow is still strongly radiation dominated because the average temperatures and densities are $3 \times 10^{7} \mathrm{~K}$ and $10^{-12} \mathrm{~g} \mathrm{~cm}^{-3}$ (except in case of models B02 and B03 where the density is much larger, although they are still radiation dominated; Fig. 12), respectively. Thus the adiabatic index in this region is very close to $4 / 3$. However, the fit of the physical variables by power laws as a function of the distance along the rotational axis produces results which are significantly different from those obtained for type-A models (Sect. 3.1.1), and which are not in agreement with the analytic estimates of Levinson \& Eichler (2000). This disagreement has two reasons. On the one hand, the interaction between the external wind medium and the relativistic outflow is strongly affected by $\mathrm{KH}$ instabilities and not restricted to a thin layer as assumed by Levinson \& Eichler 2000). On the other hand, the accretion torus in our models is thick (i.e., vertically extended) and not an infinitesimally thin ring as in the analytic treatment by Levinson \& Eichler (2000). This prevents the outflow from being sufficiently smooth to be well fitted by a single power law. 


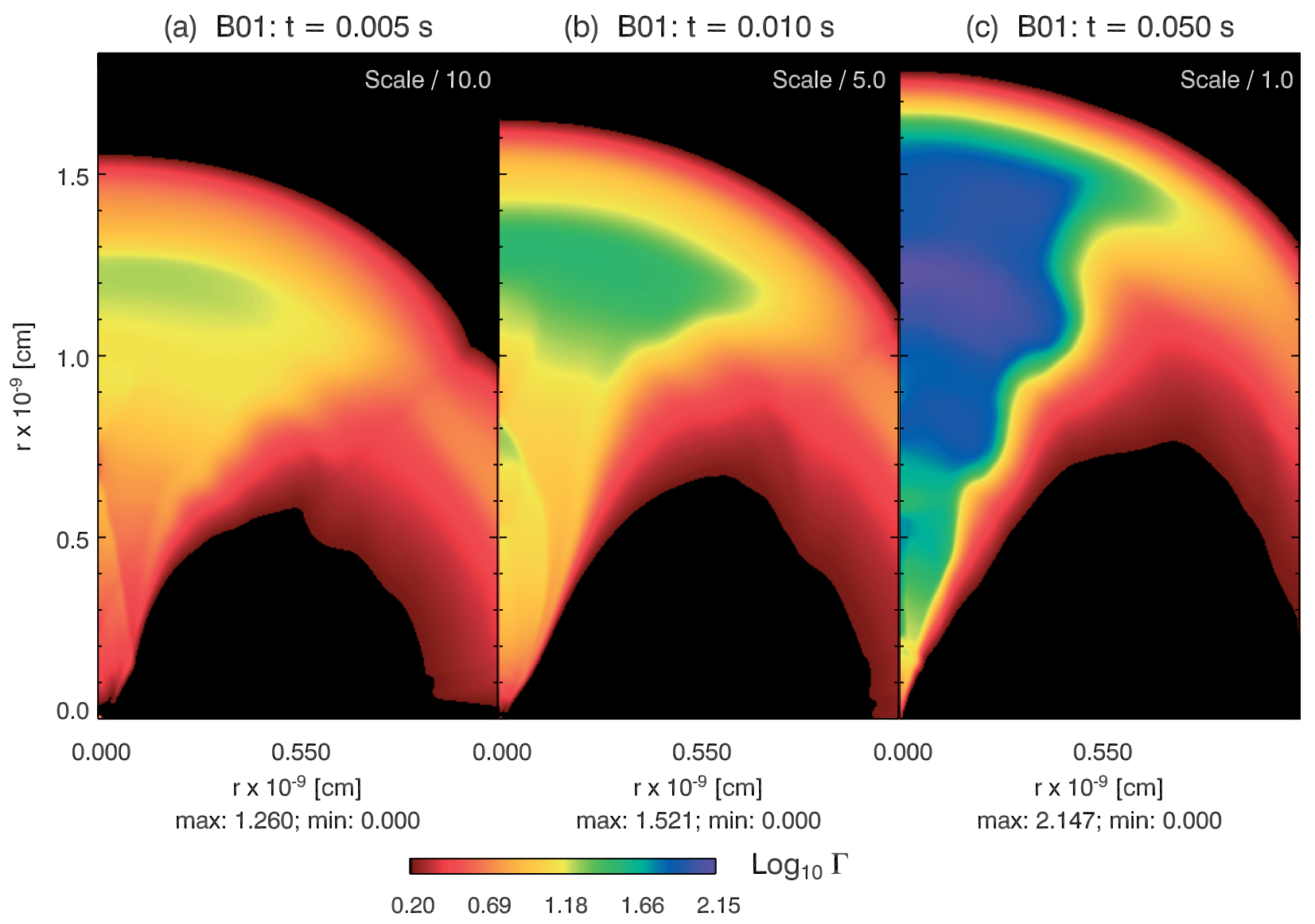

Fig. 13. Snapshots of the logarithm of the Lorentz factor of model B01 before the shut off of the energy release (the snapshot times are provided above each panel). The color scale is limited in order to enhance the details of the outflow region. Note that the distance scale has been adjusted in the two left panels in order to enlarge the details of the earlier phases of the evolution. The maximum and minimum Lorentz factor values (in logarithmic scale) of each model are given below the panels.

\subsubsection{Dependence on the total energy deposition rate $\dot{E}$}

To study the dependence of the results for models of type-B on the assumed total energy deposition rate we have fixed the half-opening angle of the deposition cone to $45^{\circ}$. The models involved in this study are B01, B04 and B05 (Table 1) with energy deposition rates of $2 \times 10^{50} \mathrm{erg} \mathrm{s}^{-1}, 10^{49} \mathrm{erg} \mathrm{s}^{-1}$ and $10^{51} \mathrm{erg} \mathrm{s}^{-1}$, respectively.

Increasing the energy deposition rate in models of type-B leads, on the one hand, to a progressive widening of the ultrarelativistic outflow and, on the other hand, to an increase of the average Lorentz factor of the fireball at all times (Fig. 15). From Table 1 we learn that the opening angle of the wind is smaller than the deposition angle $\theta_{0}$. Thus, as for models of type-A, the opening angle is not set by our choice of $\theta_{0}$, but is constrained by the presence of the thick torus.

Within an outflow crossing time of the torus in the axial direction $(\approx 0.5 \mathrm{~ms}$; note that the torus is more compact than in models of type-A, i.e., the crossing time is smaller), the fluid accelerates up to Lorentz factors of $\approx 3$, i.e., the Lorentz factor exceeds the inverse of the release angle $\left(\theta_{0}^{-1}=1.27\right)$. Hence, the sideways expansion of the outflow is drastically reduced and the initially imprinted opening angle remains almost unchanged during the later propagation of the fireball. Indeed, if the outflow is not affected by strong $\mathrm{KH}$ instabilities (as in the case of model B04), the fluid quickly enters an ultrarelativistic regime where, after $10 \mathrm{~ms}$, the maximum Lorentz factor can be larger than 30 (Table 1).

The amount of mass in the fireball (Table 1) increases linearly with $\dot{E}$. A linear fit (above the critical threshold) yields $\log M_{\mathrm{f}}=0.97 \log \dot{E}-24.2$, where $\dot{E}$ and $M_{\mathrm{f}}$ are given in cgs units. The mass mainly accumulatess in the cocoon which surrounds the outflowing fireball, and near the leading edge of the outflow. In contrast to the wind solutions found for typeA models, the density varies by up to 10 orders of magnitude over radial scales of $\approx 10^{8} \mathrm{~cm}-10^{9} \mathrm{~cm}$ within the central cone of the fireball (Fig. 12).

\subsubsection{Dependence on the half-opening angle of the energy deposition cone}

For a fixed, moderate energy deposition rate of $2 \times 10^{50} \mathrm{erg} / \mathrm{s}$, we study the influence of an increasing opening half-angle of the deposition cone from $45^{\circ}$ to $75^{\circ}$ (models B01, B02 and B03; Table 1; Fig. 15). We point out that these models also differ in the energy deposition rate per unit of volume $\left(\dot{E} / V_{\mathrm{dep}}\right)$.

Depending on the opening angle, either a highly relativistic outflow is produced (models B01 and B02 display $\Gamma_{\max } \gtrsim 240$ ), 


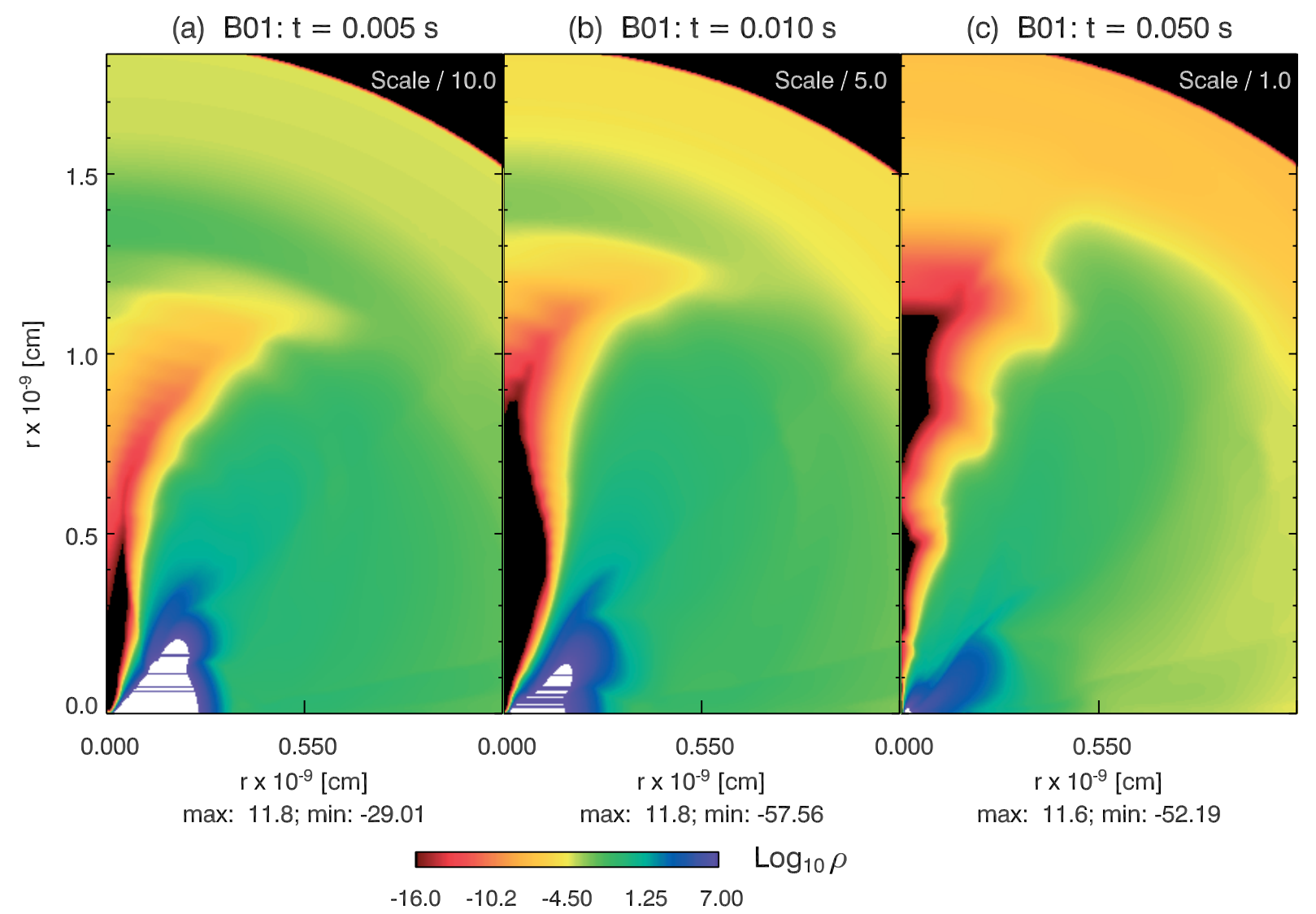

Fig. 14. Snapshots of the logarithm of the rest mass density of model B01 before the shut off of the energy release (the snapshot times are provided above each panel). The color scale is limited in order to enhance the details of the outflow region. Note that the distance scale has been adjusted in the two left panels in order to enlarge the details of the earlier phases of the evolution. The maximum and minimum density values (in logarithmic scale) of each model are given below the panels.

or only a moderately relativistic jet is generated (model B03, $\Gamma \gtrsim 10$; Fig. 15). Therefore, the value of $\dot{E} / V_{\text {dep }}$ determines whether a collimated jet (for small values) or a wide-angle jet (for larger values) is produced.

Type-B models display a moderately relativistic $(\Gamma \gtrsim 10)$ shell surrounding the central ultrarelativistic cone of the outflow, which makes it difficult to define an opening angle of the outflow. We define $\theta_{w}$ as the angle of the cone around the symmetry axis where $\Gamma>\Gamma_{\text {op }} \equiv 10$. Using values of $\Gamma_{\text {op }}$ in the interval [10-25] does not lead to substantially different values of $\theta_{w}$ (except for model B03 where the maximum value of $\Gamma$ is $\Gamma_{\max }=17.22$ ). With this definition, and for a fixed, moderate total energy deposition rate the opening angle of the outflow $\theta_{w}$ is sensitive to an increase of the deposition angle $\theta_{0}$ (Table 1 ) because the layer of the outflow between $\approx 17^{\circ}$ and $\approx 35^{\circ}$ enclosing the central relativistic or ultrarelativistic cone is mostly filled with matter which was pulled up and accelerated from that part of the torus overlapping with the deposition region. In the three models under consideration the deposition region extends beyond $35^{\circ}$. The energy per unit of mass released in the above mentioned layer is sufficient to speed up the fluid beyond $\Gamma \gtrsim 100$, however only in models B01 and B02.

After $100 \mathrm{~ms}, \theta_{w}$ ranges from $2.3^{\circ}$ (model $\mathrm{B} 03$ ) to $30^{\circ}$ (model B01), the values decreasing with time (Table 1). In models B01 and B02 this decrease is caused by the change from a relativistic to an ultrarelativistic flow due to the continuing deposition of energy. When the fluid speed in the radial direction approaches the speed of light the transverse velocity component can only be very small, i.e., the sideways expansion is largely suppressed. In case of B03, a highly collimated jet forms very early (in less than $20 \mathrm{~ms}$ ), and after this wind-to-jet transition the opening angle of the outflow remains almost constant. Different from type-A models the transition is not caused by the inertial confinement due to the external medium, but by the growth of KH instabilities which tend to pinch the conical channel. These KH instabilities are of much larger amplitude for models with large $\theta_{0}$, because the over-pressure generated by the release of energy is smaller, i.e., more torus matter is entrained into the outflow. Indeed, the energy in the ultrarelativistic outflow and the mass of the fireball $M_{\mathrm{f}}$ tends to decrease with increasing $\theta_{0}$. Since the entrained mass is not homogeneously distributed but localized towards the lateral edges of the fireball, in these regions the Lorentz factor of the outflow decreases below 10. Therefore, they are not included in our computation of the fireball mass (Table 1). The process of mass entrainment is extreme in the case of model B03, where for most of the outflow mass $\Gamma<10$ holds.

Type-B models produce radiation dominated, wide-angle, shocked jets (see the profiles of the density, pressure and Lorentz factor in Fig. 12). Since they are radiation dominated 


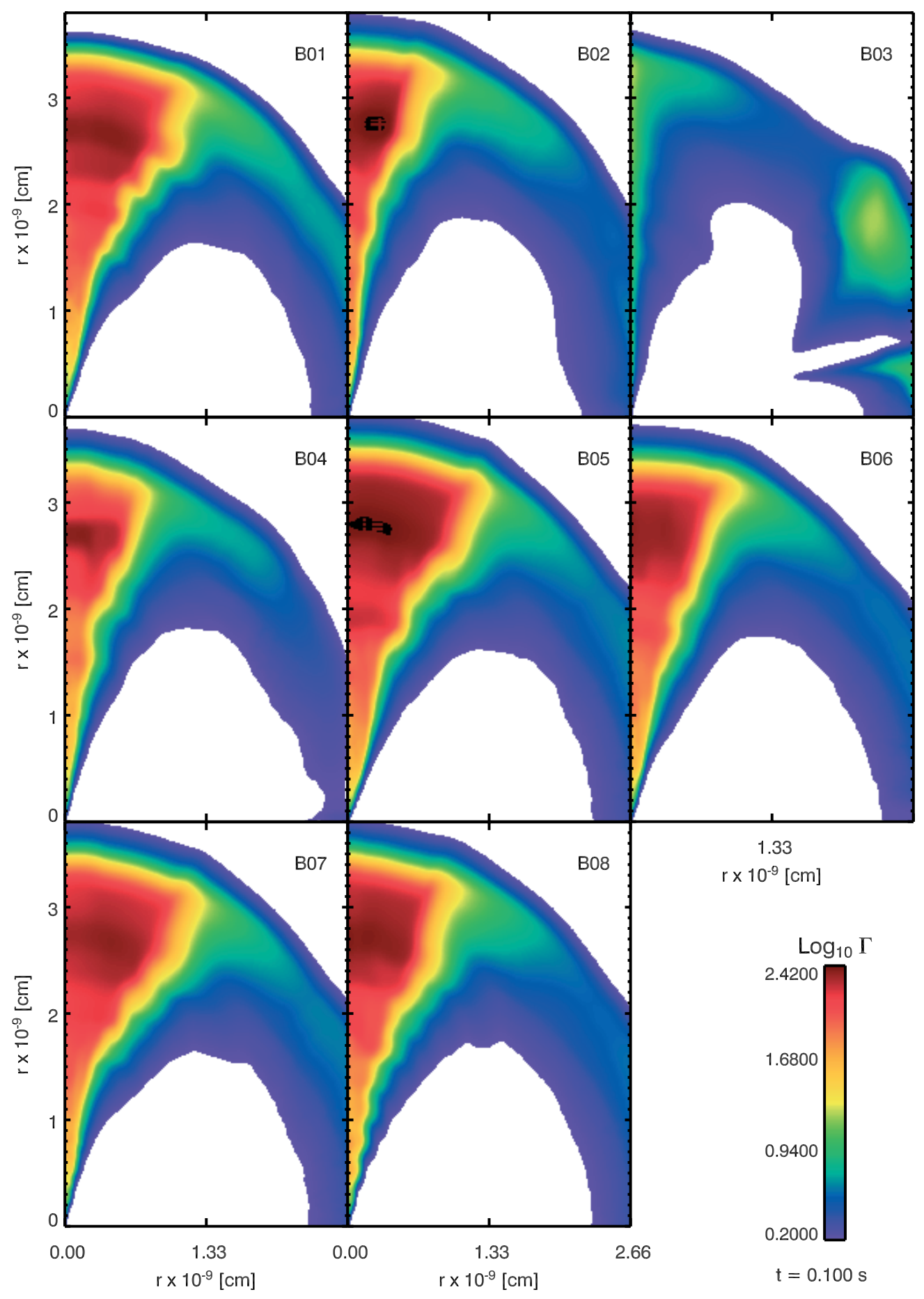

Fig. 15. Logarithm of the Lorentz factor of the models of type-B after $0.1 \mathrm{~s}$ of their evolution. The color scale is limited in order to enhance the details of the outflow region.

the pressure variations effectively decouple from the density fluctuations (Aloy et al. 2002). While the radiation pressure displays rather small variability across and along the fireball and falls almost monotonically about 15 orders of magnitude in the radial direction, the density variation in the same direction is more than 50 orders of magnitude and clearly non-monotonic (Fig. 12). The temperature of the fireball behaves similar as the pressure but with even less variability. The Lorentz factor, which also shows a non-monotonic increase with distance, is modulated by a number of shocks whose shape is either biconical (up to $\approx 10^{9} \mathrm{~cm}$ ) or radial (at larger distances). As a result of the non-monotonic behaviour, the outflow of type-B models cannot be properly fitted by power laws as in case of type-A models. The cause of this difference is easy to understand. The outflow of type-B models is much less dense than that of type-A models. Thereby, the entrainment of high density matter from the torus produces larger relative density perturbations than in type-A models. 


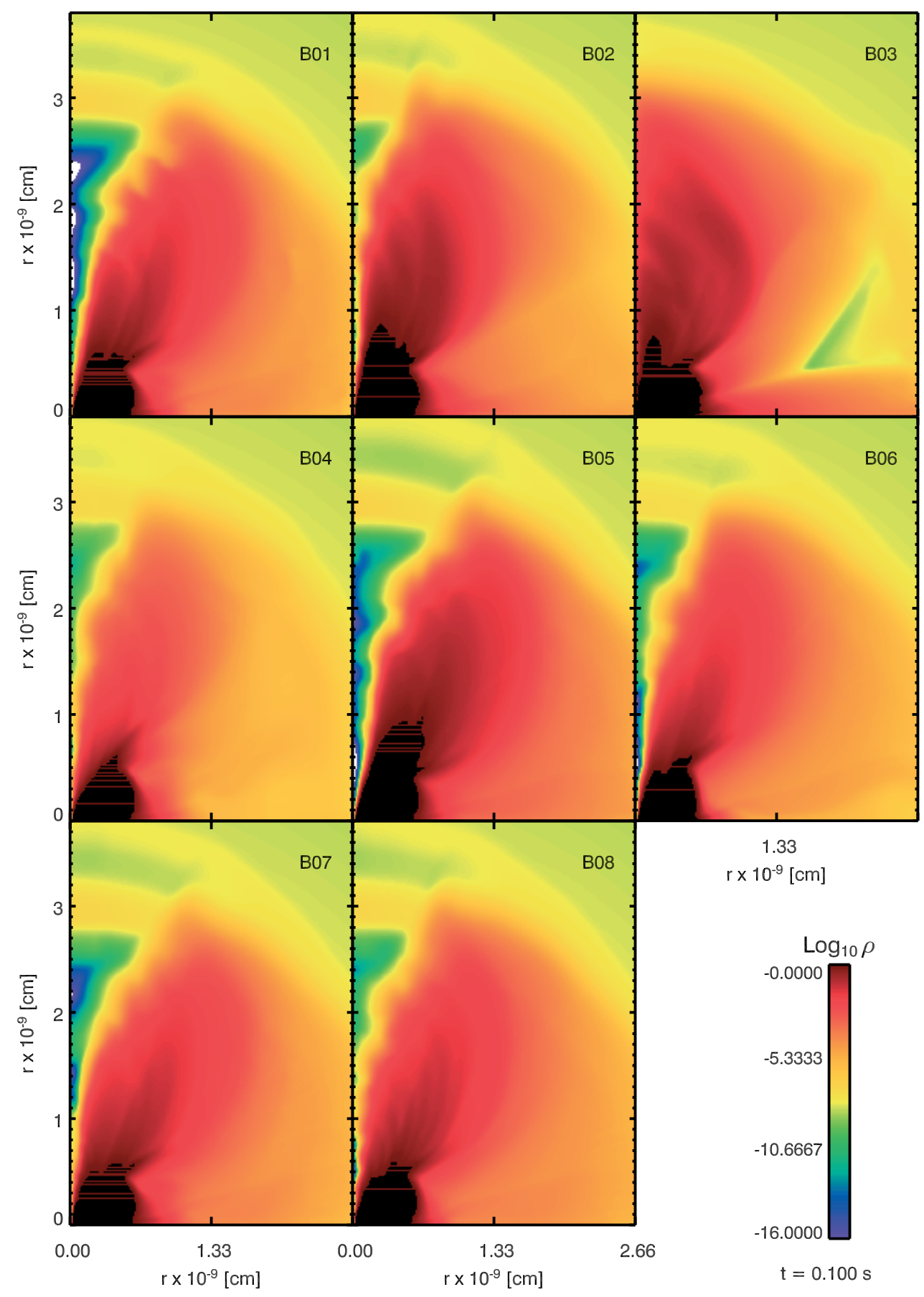

Fig. 16. Logarithm of the rest-mass density (in $\mathrm{g} \mathrm{cm}^{-3}$ ) of the models of type-B after $0.1 \mathrm{~s}$ of their evolution. The color scale is limited in order to enhance the details of the outflow region.

The differences between models of type-B and type-A (and also the theoretical models of Levinson \& Eichler 2000) are particularly large in the radially outer part of the fireball, while in the unshocked region the two types of models differ much less. This is expected because, on the one hand, the shocked region is neither included in simplified theoretical considerations nor can it be fitted by power-laws. On the other hand, the exact scaling laws of the outflow depend strongly on the details of the interaction between the surface of the fireball and the external medium. While this interaction is assumed by Levinson \& Eichler (2000) to be confined to a thin layer, we find that it takes place in an angularly extended region where large-amplitude $\mathrm{KH}$ instabilities occur.

As in case of type-A models (see Sect. 3.1.2) the theory of Levinson \& Eichler (2000) is also not suitable to describe the collimation of the ultrarelativistic outflow obtained in our type-B models, where the presence of the torus is responsible for the jet collimation, too. This fact is supported by the results shown in Fig. 17 where the values of the half-opening angle $(\alpha)$ of the ultrarelativistic outflow are displayed calculated according to the simplified analytic model of Levinson \& Eichler (2000), which assumes that an external, baryonic wind is 


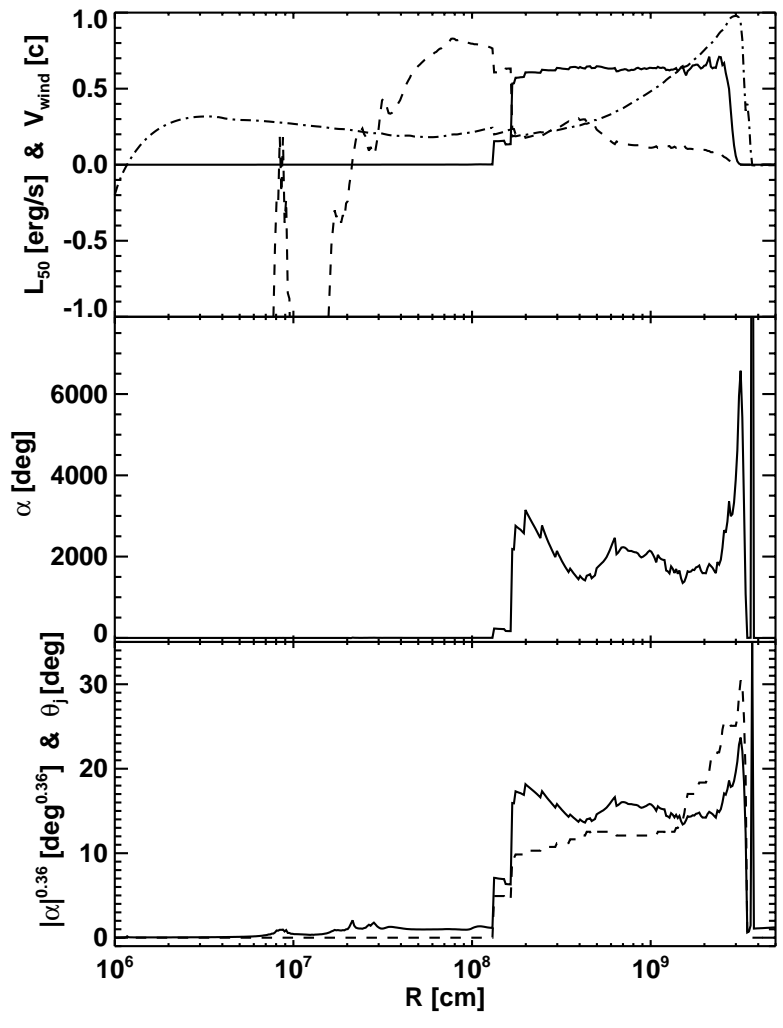

Fig. 17. The top panel shows the luminosity of the ultrarelativistic fireball (solid line) and of the surrounding moderately relativistic wind (dashed line) in units of $10^{50} \mathrm{erg} \mathrm{s}^{-1}$, as well as the angularlyaveraged, radial velocity of the wind $v_{w} / c$ (dashed-dotted line) as a function of the radial distance for model B01 at $t=0.1 \mathrm{~s}$. The middle panel shows the expected fireball half-opening angle (in degrees) as predicted by the analytic model of Levinson \& Eichler (2000), i.e., $\alpha=\pi \beta_{w}^{-1} \Gamma_{\mathrm{fo}}^{-2}\left(L_{\mathrm{f}} / L_{w}\right)$, where $\beta_{w}=v_{w} / c$ is the velocity of the surrounding wind medium $\left(v_{w}\right)$ in units of the speed of light $(c), \Gamma_{\mathrm{fo}}$ is the Lorentz factor at the base of the jet (which is of order unity in all our simulations), and $L_{\mathrm{f}}$ and $L_{w}$ are the luminosities of the fireball and of the external wind, as defined in the text. For the calculation of $\alpha$, the angularly-averaged, radial velocity $v_{w}$ of the external wind medium (see top panel) is computed at all radii $R$. Finally, the bottom panel shows $|\alpha|^{0.36}$ as a function of the radius (solid line) compared with the local half-opening angle of the fireball $\theta_{j}$ as a function of the radius (dashed line). The value of $\theta_{j}$ is computed as the maximum polar angle where, at a distance $R$, the Lorentz factor of the fluid is larger than 10 . Note that the fireball half-opening angle $\theta_{w}$ (given in Tables 1 and 2) is defined as the maximum of all the local half-opening angles $\theta_{j}$.

responsible for the collimation of the jet: $\alpha \approx \pi \beta_{w}^{-1} \Gamma_{\mathrm{fo}}^{-2}\left(L_{\mathrm{f}} / L_{w}\right)$, where $\beta_{w}=v_{w} / c, v_{w}$ is the velocity of the wind, $\Gamma_{\mathrm{fo}}$ is the Lorentz factor at the base of the jet $\left(\Gamma_{\text {fo }} \approx 1\right.$ in all our simulations), and $L_{\mathrm{f}}$ and $L_{w}$ are the luminosities of the fireball and of the external wind, respectively. These luminosities are evaluated as $L_{\mathrm{f}}=2 \pi R^{2} c^{3}\left(1-2 R_{\mathrm{g}} / R\right)^{3 / 2} \int_{0}^{\theta_{w}(R)} \mathrm{d} \theta \sin ^{2} \theta \rho \Gamma \beta_{r}(h \Gamma-1)$ and $L_{w}=2 \pi R^{2} c^{3}\left(1-2 R_{\mathrm{g}} / R\right)^{3 / 2} \int_{\theta_{w}(R)}^{\pi / 2} \mathrm{~d} \theta \sin ^{2} \theta \rho \Gamma \beta_{\mathrm{r}}(h \Gamma-1)$, respectively. In these expressions, $h$ is the specific enthalpy and $\beta_{\mathrm{r}}=v_{\mathrm{r}} / c$ with $v_{\mathrm{r}}$ being the radial velocity. For this quantitative comparison we identify the moderately relativistic outflow with $\Gamma<10$ around the fireball with the baryonic wind which produces the jet collimation in Levinson \& Eichler's model.
We further assume that the velocity of the wind $v_{w}$ can be approximated at each radial distance $R$ by the angularly-averaged, radial velocity of the external medium. The latter velocity is computed as the arithmetic mean of the radial velocities of all cells at a distance $R$ in the interval between $\theta_{w}(R)$ (the polar angle at which the Lorentz factor becomes smaller than 10 at a radial distance $R$ ) and $\pi / 2$.

Comparing the values of the fireball half-opening angle shown in Table 1 with the huge (meaningless) values shown in the bottom panel of Fig. 17, we conclude that the nonrelativistic wind is clearly not responsible for the fireball collimation. We point out that the value of $\alpha$ is not unique. It changes by a factor of $\sim 3$ depending on the radial distance at which it is computed. A similar variability is also found for the local half-opening angle of the fireball, $\theta_{j}$, calculated as the maximum polar angle where, at a distance $R$, the Lorentz factor of the fluid is larger than 10 (Fig. 17 bottom panel). We have found that the function $|\alpha|^{0.36}$ reasonably fits $\theta_{j}$.

\subsubsection{Dependence on the deposition volume $V_{\text {dep }}$ and on the total energy deposition rate $\dot{E}$ for $\dot{E} / V_{\text {dep }}=$ constant}

In two models of type-B we have varied the half-opening angle of the energy deposition cone simultaneously with the total energy deposition rate $\dot{E}$, while keeping the rate of energy deposition per unit of volume $\dot{E} / V_{\text {dep }}$ constant. The two corresponding models are $\mathrm{B} 02$ (with a large energy deposition rate and deposition angle $\theta_{0}=60^{\circ}$ ) and B06 (with a smaller deposition rate and $\theta_{0}=41.4^{\circ}$ ).

Both models develop a wide-angle ultrarelativistic jet because the lateral edges of the deposition region and, further downstream, also the lateral boundaries of the outflow are causally connected, i.e., KH modes can grow modulating the variation of the physical variables inside the outflow. Both models show similar global properties (propagation speed of the fireball, opening angle of the wind, mass of the fireball) during the time when energy is deposited (Table 1). A more detailed comparison reveals minor differences, for example, in the maximum Lorentz factor (larger in model B02), the propagation velocity (larger in B02), and the fireball opening angle after a canonical time of $100 \mathrm{~ms}$ (smaller in B02 than in B06, although in both cases $\theta_{w} \approx 22^{\circ}$; see Table 1 ). The model with the smaller deposition angle (B06) sweeps up less mass from the external medium, and drags less mass off the torus (the deposition region overlaps with the torus by $\approx 16^{\circ}$ ) than the model with the larger deposition angle and deposition rate (the energy deposition cone overlaps with the torus by $\approx 35^{\circ}$ in B02). This explains why the outflow in the latter model is more massive $\left(M_{\mathrm{f}}^{B 02}=2.5 \times 10^{24} \mathrm{~g}\right)$ than in model B06 $\left(M_{\mathrm{f}}^{B 06}=1.4 \times 10^{24} \mathrm{~g}\right)$.

Looking at the density distribution (Fig. 16) we see that the ultrarelativistic region of model B02 is denser (on average) and displays much smaller density fluctuations than model B06 (Fig. 12, top panel). Furthermore, model B06 develops a more extended unshocked region (Fig. 12), where the Lorentz factor is larger than that of model B02 after an evolutionary time of $100 \mathrm{~ms}$ (Fig. 12, bottom panel). The propagation velocity, 


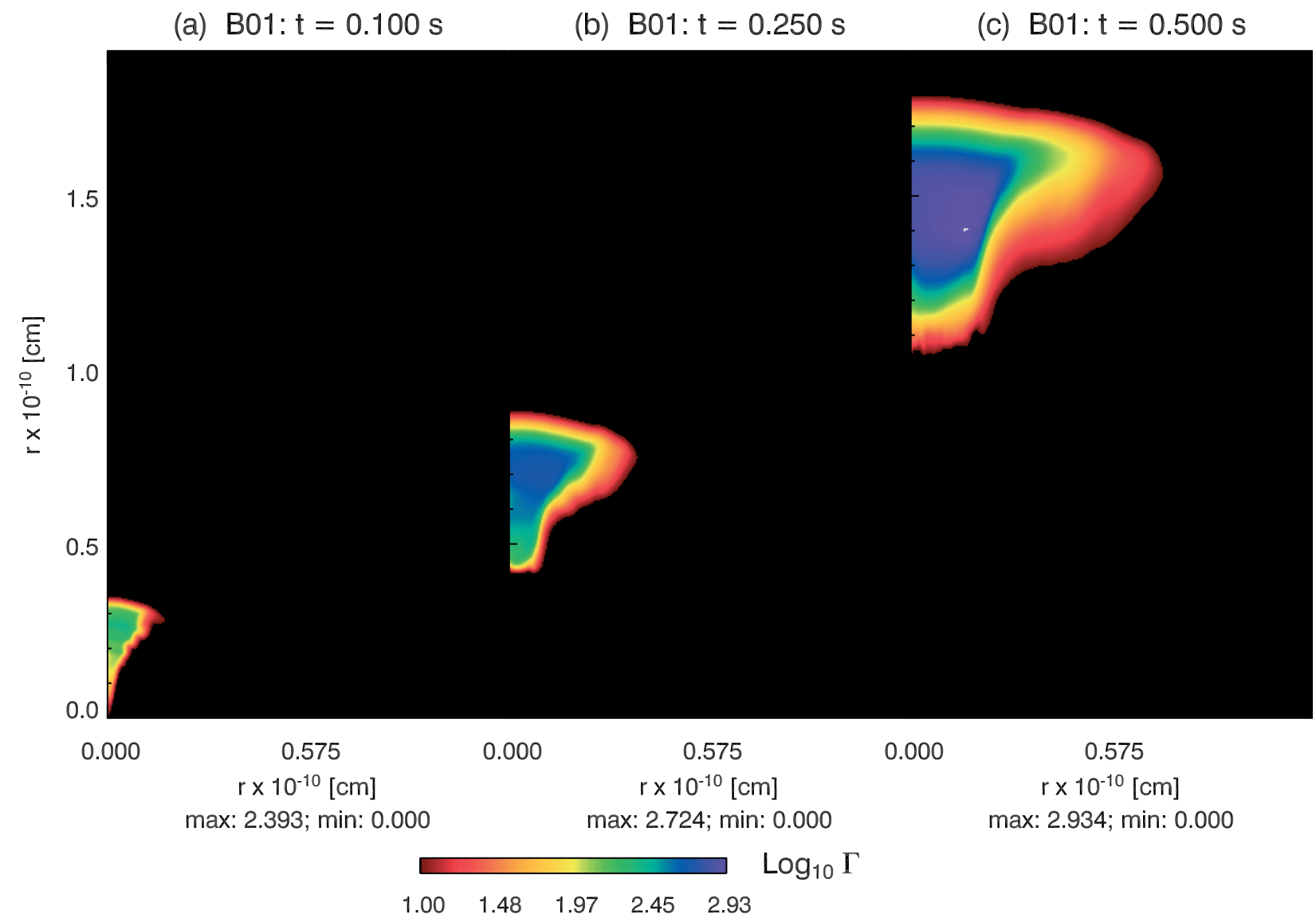

Fig. 18. Evolution of the logarithm of the Lorentz factor of model B01 after the shut down of the central energy supply. Time is measured from the moment when the energy deposition was started.

although very similar, is systematically smaller in model B06. The differences between the two models arise from the larger angular extent of the deposition region in model B02. Since the deposition region overlaps more with the torus in model B02 than in model B06 there is more mass pulled up from the torus in the former model. This explains the larger density and, as a result, the smaller fluid Lorentz factor in the shocked region of the fireball.

\subsubsection{Dependence on the time variability of the energy source}

In models B07 and B08 we have varied the energy deposition rate according to the prescriptions given in Eqs. (10) and (11). The energy released in models B07 and B08 until time infinity is the same as in model B01 during $100 \mathrm{~ms}$. The width of the deposition region is also equal to that of model B01. With both models we try to determine the consequences of an intrinsic source variability as compared to the variability caused by the interaction with the accretion torus and the merger halo.

After $100 \mathrm{~ms}$ of continuous deposition of energy, the decay of the energy deposition rate of model B07 for $t>0.03 \mathrm{~s}$ has not produced any obvious signature in the Lorentz factor of the outflow, which in fact looks similar as in model B01 (Fig. 15). Even the variations of Lorentz factor in the outflow are almost indistinguishable between the model with constant energy release and the models with a variable, burst-like deposition of energy. However, the ultrarelativistic central core of the outflow of model B07 is (on average) 50\% denser than that of model B01 (Fig. 16), which is a consequence of a larger mass entrainment resulting from the smaller $\dot{E} / V_{\text {dep }}$ near the system axis after $\sim 35 \mathrm{~ms}$ relative to model B01.

The periodic variation of the energy deposition rate yields a modulated growth of the Lorentz factor of model B08 the periodicity following that of the energy release (in particular up to distances of $\sim 2 \times 10^{9} \mathrm{~cm}$, where the grid resolution starts to become too coarse, and along the boundaries of the ultrarelativistic core of the fireball; Fig. 15). Model B08 also has a larger mass entrainment than model B01 for the reason mentioned above. It is slightly larger than in model B07 because of the sinusoidal variation of the deposition rate: $\dot{E} / V_{\text {dep }}$ of model B08 is $50 \%$ smaller than that of model B07 during the epochs of minimum energy release (i.e., when $\sin \left(2 \pi t / t_{\text {var }}\right)=-1$ ), when a larger amount of mass is entrained. Once that mass is inside of the fireball, it is never expelled again.

\section{Evolution after the switch-off of the central energy source}

In the previous sections we have discussed the evolution of the relativistic outflow that forms by the release of thermal energy around a black hole-accretion torus system. The simulations were carried on for a canonical time of $100 \mathrm{~ms}$ 


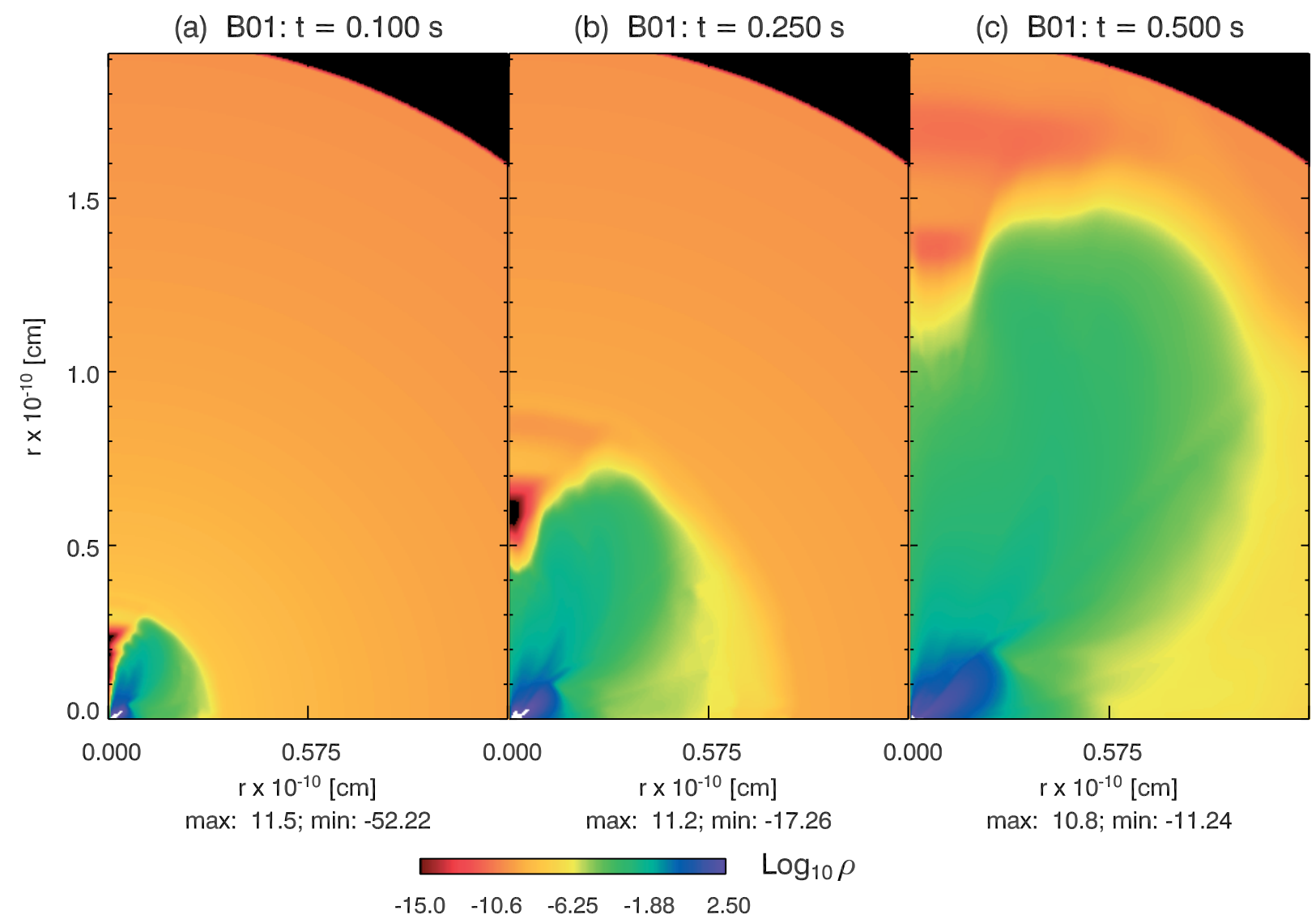

Fig. 19. Evolution of the logarithm of the rest-mass density (in $\mathrm{g} \mathrm{cm}^{-3}$ ) of model B01 after the shutdown of the central energy supply. Time is measured from the moment when the energy deposition was started.

during which the source was assumed to release energy either at a constant rate (models B01 to B06) or with a rate decaying with time (models B07 and B08) as $t^{-3 / 2}$ (see Sect. 3.2). After $100 \mathrm{~ms}$, the different fireballs have reached a distance of $\simeq 3 \times 10^{9} \mathrm{~cm}$. However, according to the standard model, the GRB phenomenon can be produced only at radial distances of $\gtrsim 10^{13} \mathrm{~cm}$ from the energy source, i.e., the outflow must still propagate and persist over another 4 orders of magnitude in radius before an observable signal emerges. In order to investigate longer timescales than the epoch of energy release, we have followed the evolution of the type-B models for another $400 \mathrm{~ms}$ after the central energy supply had either been shut off (B01 to B06) or during which it continued at a decreasing rate (models B07 and B08).

In the following sections we will concentrate on the evolution of the prototype model B01 (Sect. 4.1), and afterwards we will outline the particularities of the other type-B models (Sect. 4.2).

\subsection{Model B01}

After the epoch of $100 \mathrm{~ms}$ of a constant rate of energy release has ended (except for the first $10 \mathrm{~ms}$ during which the energy deposition rate is linearly increased), the high-Lorentz factor outflow possesses a conical shape which decouples from the energy deposition region, and moves away from the black hole (Fig. 18). The shape is mainly a consequence of the continuing radial acceleration of the matter in the outflow. The angular structure of the adiabatically expanding fireball is non-uniform because of a larger baryon loading at larger polar angles. The Lorentz factor reaches values between 20 and 50 (approximately, between $\approx 15^{\circ}$ and $30^{\circ}$ off axis) where the fireball is loaded with baryons extracted from the outermost layers of the torus. These interaction layers have still mildly relativistic or subrelativistic velocities at the time the energy release is switched off. In contrast, the ultrarelativistic core has swept up only matter from the dilute halo. One, therefore, finds variations of the Lorentz factor (Fig. 18) which depend on the lateral angle (in addition to the radial variations of $\Gamma$ ). The maximum Lorentz factor $\left(\Gamma_{\max } \simeq 850\right)$ is attained close to the lateral edges of the fireball core (at polar angles around $8^{\circ}$ ) and in its rear part, while in the region around the axis $\left(\theta<3^{\circ}\right)$ the fluid Lorentz factor is about $20 \%$ smaller than its maximum value (Fig. 21). For small angles, the Lorentz factor exhibits a fast rise from the backward end of the fireball to its center where values beyond 800 are found (Fig. 20). From this local maximum the Lorentz factor decreases slightly towards the terminal shock of the wind (see the violet shade in the right panel of Fig. 18). For larger angles $\left(\theta>15^{\circ}\right)$ the radial profile of the Lorentz factor is similar although the values here are much smaller $(\Gamma \simeq 30)$. This radial profile of the Lorentz factor is the result of the beginning of a coasting phase, that starts soon after the energy release ceases. 


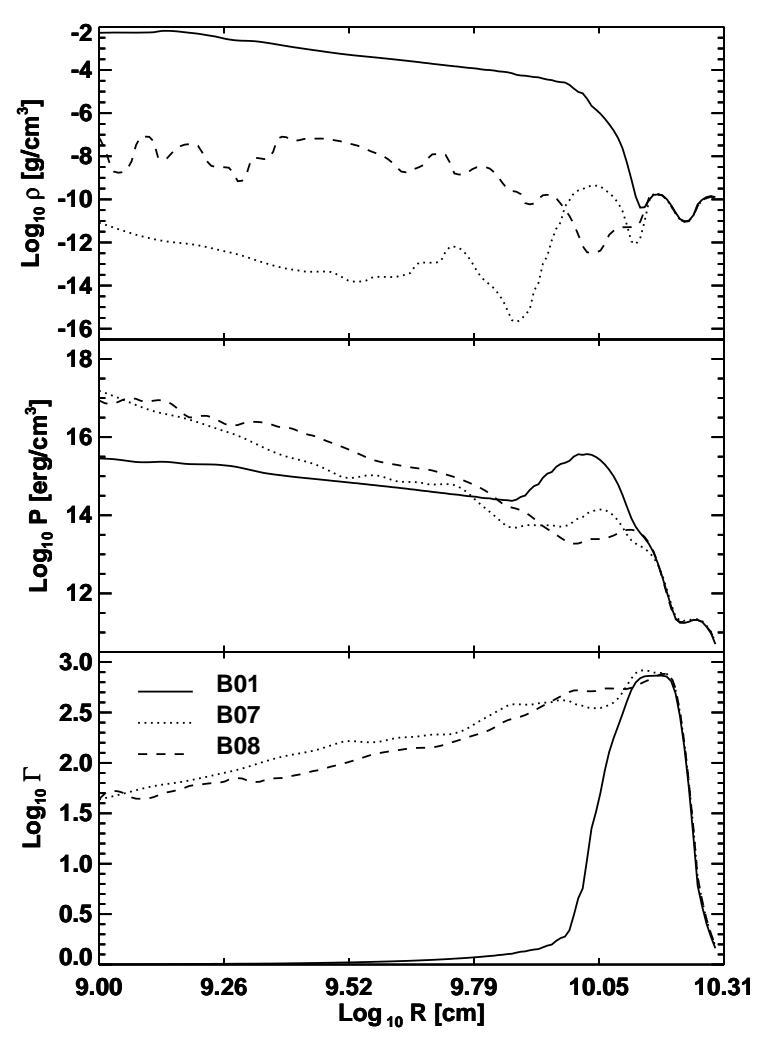

Fig. 20. Logarithm of the rest-mass density (top panel), of the pressure (middle panel) and of the fluid Lorentz factor (bottom panel) vs. radius along the symmetry axis for models B01, B07 and B08 (see legend in the plot) after $500 \mathrm{~ms}$.

The rest-mass density (Fig. 19) in the fireball does not preserve the irregular structure imprinted by the $\mathrm{KH}$ instabilities that had developed near the lateral edges during the earlier evolution. After the shutdown of the energy deposition, two voids (density minima) show up in the central part of the fireball, one close to its rear part and another one close to the front of the fireball (solid line in Fig. 20). The two voids are radially separated by a 20 times denser "wall", corresponding to a reverse shock that sweeps backwards into the fireball.

The pressure in the fireball decreases with increasing radial distance and increasing polar angle. Across the fireball, in the polar direction, the pressure is lowest close to the side edges of the fireball core and is highest around the symmetry axis (where the density is about a factor of 2 larger than at the sides; Fig. 21). The pressure variation in the radial direction can be as large as three orders of magnitude (Fig. 20). However, due to the fact that the fireball is radiation dominated, the variation of pressure (or temperature) is much smaller than that of the rest-mass density. The structure of the outflow can be understood by the combination of three main dynamical effects. First, there is a trend to adopt a radially stratified wind structure due to the expansion of the outflow as it moves radially outwards. This explains the decrease of the pressure, temperature and density (and the increase of the Lorentz factor) in the radial direction. Second, there are local variations of the physical variables caused by the $\mathrm{KH}$ instabilities during the early interaction of the fireball with the external medium and, particularly,

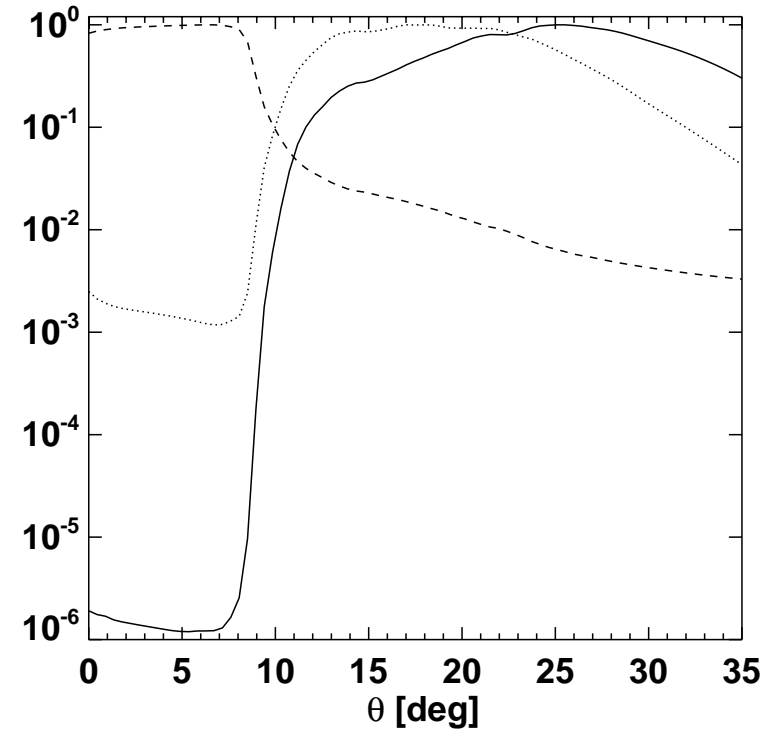

Fig. 21. Lorentz factor (dashed line), pressure (dotted line) and restmass density (solid line) in polar direction at a radial distance of $1.4 \times$ $10^{10} \mathrm{~cm}$ after $500 \mathrm{~ms}$ of evolution of model B01. The quantities are normalized to their respective maxima in the displayed angular interval. Note the rarefaction visible between the center and the edges of the fireball $\left(\right.$ at $\left.\approx 8^{\circ}\right)$.

with the torus. The largest of these instabilities survive beyond the shutdown of the energy deposition. Most of the small scale variations are (numerically) suppressed because of the logarithmic coarsening of the grid in the radial direction. However, $\mathrm{KH}$ modes with wavelengths smaller than the width of the thick, expanding layer surrounding the central ultrarelativistic cone are also physically damped (Birkinshaw 1991). Third, there is a genuinely relativistic effect (Pons et al. 2000; Rezolla \& Zanotti 2002) that leads to the generation of a rarefaction wave and a shock (instead of two shocks) for collisions between fluids in case of a small impact angle (an example of this phenomenon in the context of parsec scale jets can be found in Aloy et al. 2003). The effect arises in the presence of a non-vanishing tangential velocity component at a relativistic shock. The generalized jump conditions require that the quantity $h \Gamma v_{\mathrm{t}}$ is equal (where $h$ and $v_{\mathrm{t}}$ are the specific enthalpy and the velocity component tangential to the shock front, respectively) on both sides of the shock. The rarefaction propagates from the collision region into the medium that has smaller density and higher specific enthalpy. The shock moves in the opposite direction into the fluid that has larger density and smaller specific enthalpy.

This described situation applies to our models when the fireball expands sideways (i.e., in the lateral direction) and interacts with the external medium and the torus. Tangential velocity components are present due to the radial fluid motion in the fireball, the moderately relativistic rotation of the torus, and the expansion of the nonrelativistic ejecta that are blown off the torus surface due to the energy deposition. The interaction leads, on the one hand, to the formation of a rarefaction wave that starts from the lateral edges of the fireball, propagates very slowly towards the axis, and leaves behind a region with negative angular $(\theta-)$ velocities (i.e., velocities directed to the axis). On the other hand, the interaction generates a shocked layer 

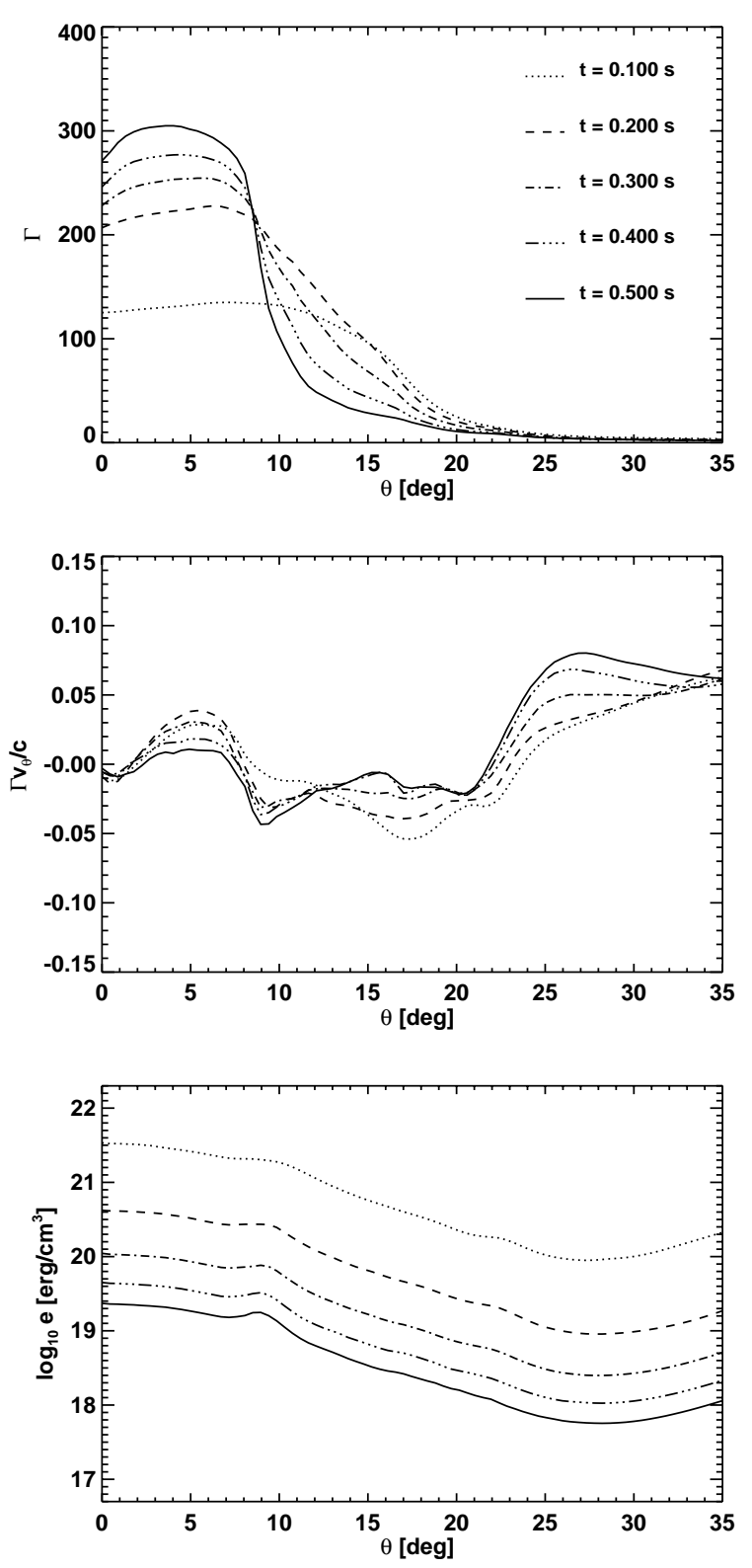

Fig. 22. Radially averaged values of the Lorentz factor (top panel), angular component of the four-velocity (intermediate panel) and energy density (bottom panel) as a function of the polar angle for model B01. The different lines correspond to different moments in time spaced by $100 \mathrm{~ms}$ (the times are listed in the top panel).

that encloses the fireball's side edges. Because of the rarefaction wave the pressure near the edges of the fireball core (which is a region of low density and high specific enthalpy) is smaller than near the axis (in Fig. 21, the rarefaction extends from $\approx 7^{\circ}$ to $\approx 14^{\circ}$ ). Analytic modeling of the collision of the fireball with the external medium in two dimensions (assuming planar symmetry) shows that a rarefaction wave forms instead of a shock when the impact angle is smaller than $\sim 35^{\circ}$.

We point out that the existence of such a rarefaction wave was included neither in analytic models - Levinson \& Eichler (2000) assumed that a thin shocked layer that results from the propagation of two oblique shocks - nor in phenomenological fireball-jet models assumed to be either homogeneous (e.g.,
Piran 1999, 2000) or structured (e.g., Kumar \& Granot 2003; Salmonson 2003).

From the above discussion it is clear that the ultrarelativistic outflow is laterally inhomogeneous and not bounded by sharp edges (as assumed in the so-called homogeneous or top-hat jet models). The outflow profile can also not be fitted by a monotonic function (a power-law or a Gaussian function as assumed in the structured jet models). Let us now consider the one-dimensional angular outflow structure by calculating the radial average of the quantities displayed in Fig. 22. We apply the formula $\bar{A}=\left(\Delta V_{r}\right)^{-1} \int_{R_{\min }}^{R_{\max }} r^{2} A \mathrm{~d} r$, with $\Delta V_{r}=$ $\left(R_{\max }^{3}-R_{\min }^{3}\right) / 3, R_{\max }$ and $R_{\min }$ being the radii of the front and of the rear end of the relativistic outflow, respectively. We find that the evolution of the angular profiles is rather different from the behaviour discussed by Kumar \& Granot (2003). The angular wedge with high Lorentz factors becomes narrower with time and the angular location of the peak Lorentz factor moves to smaller angles. At the same time the peak value increases and becomes larger than the value on the symmetry axis (Fig. 22). A good fit for the radially averaged Lorentz factor $(\bar{\Gamma})$ as a function of the polar angle in the interval $\left[0^{\circ}, 90^{\circ}\right]$ is given by the expression

$$
\bar{\Gamma}=1+\frac{a_{0}+a_{1} x+a_{2} x^{2}+a_{3} x^{3}}{a_{4}+a_{5} x+\exp \left(a_{6} x\right)} .
$$

The values of the seven coefficients $a_{i}$ and the $\chi^{2}$ of the fit are listed in Table 3 for the type-B models at a time of $0.4 \mathrm{~s}$ after the shutdown of the central energy source. Attempts to fit simpler functions in a narrower interval (namely, $\left[0^{\circ}, 20^{\circ}\right]$ ) were unsuccessful. In particular, a Gaussian profile of the form $a \mathrm{e}^{b x^{2}}+1$, or a quadratic ansatz of the form $a /\left(b+c x^{2}\right)$ had to be rejected because the resulting $\chi^{2}$ values are 176 and 860 , respectively.

Figure 22 shows that during the last $0.4 \mathrm{~s}$ the increase of the averaged Lorentz factor per unit of time in a $10^{\circ}$ cone around the axis is much smaller than during the first $0.1 \mathrm{~s}$. However, we also see that the averaged Lorentz factor has not yet converged to an asymptotic value after $0.5 \mathrm{~s}$, i.e., acceleration continues. Table 2 gives a rough estimate of the maximum bulk Lorentz factor (defined in the table caption) that can be asymptotically reached by the fireball when its whole internal energy is converted into kinetic energy (provided there is no further acceleration or deceleration due to the presence of a pressure gradient in the ambient medium of swept-up matter, and that the medium at larger distances from the fireball origin is uniform and very diluted). The values of $\Gamma_{\infty}$ are still $\sim 2$ times larger than the corresponding values of $\Gamma_{\max }$ except for model B03 (Table 2). This is consistent with our hypothesis according to which the value of $\Gamma_{\infty}$ sets an upper limit to the maximum bulk Lorentz factor of the outflow. Furthermore, the fact that $\Gamma_{\max }<\Gamma_{\infty}$ suggests that there is still room for additional acceleration of the fireball fluid.

Profiles of the radially averaged energy density $(\bar{e})$ can be fitted as a function of the polar angle, in the interval $\left[0^{\circ}, 90^{\circ}\right]$, by polynomials of the form

$\bar{e}=\sum_{i=0}^{4} b_{i} x^{i}$ 
Table 2. Type-B progenitor models. The first column gives the model name, and the second one the total energy $E_{\mathrm{d}}$ released in two hemispheres during the simulated time. Columns 3 to 6 display the ratio of the total energy (internal plus kinetic) in the region of the outflow that has a Lorentz factor larger than a certain threshold value (given by the subscript of the energy in each column) relative to the energy that is released (in one hemisphere). The seventh column provides the fraction of the deposited energy (per hemisphere) that is converted into kinetic energy of the outflow with Lorentz factor larger than 100. Columns 8 to 10 show the maximum Lorentz factor $\Gamma_{\max }$, the half-opening angle $\theta_{w}$ and the mass of the fireball $M_{\mathrm{f}}$, respectively. The half-opening angle of the fireball is computed as the maximum $\theta$-coordinate of all computational cells where the fluid has a Lorentz factor above 10 and positive velocities. The mass of the fireball is computed by adding up the mass in all computational cells that match the same criterion as the one used to compute $\theta_{w}$. The last column displays an estimate of the asymptotic value of the Lorentz factor of the outflow computed as $\Gamma_{\infty}=E_{\Gamma>10} /\left(M_{\mathrm{f}} c^{2}\right)$, i.e., assuming that all internal energy is eventually converted into kinetic energy, and no mass is subsequently swept up from the environment. All the data in the table correspond to a time of evolution of $0.5 \mathrm{~s}$.

\begin{tabular}{lcccccccccc}
\hline \hline Model & $E_{\mathrm{d}}[\mathrm{erg}]$ & $\frac{E_{\Gamma>100}}{E_{\mathrm{d}}}$ & $\frac{E_{\Gamma>50}}{E_{\mathrm{d}}}$ & $\frac{E_{\Gamma>10}}{E_{\mathrm{d}}}$ & $\frac{E_{\Gamma>2}}{E_{\mathrm{d}}}$ & $\frac{E_{k, \Gamma>100}}{E_{\mathrm{d}}}$ & $\Gamma_{\max }$ & $\theta_{w}$ & $M_{\mathrm{f}}[\mathrm{g}]$ & $\Gamma_{\infty}$ \\
\hline B01 & $2 \times 10^{49}$ & 0.29 & 0.36 & 0.59 & 0.70 & $4.4 \times 10^{-3}$ & 859 & $24^{\circ}$ & $7.4 \times 10^{24}$ & 1765 \\
B02 & $2 \times 10^{49}$ & 0.09 & 0.15 & 0.35 & 0.48 & $4.8 \times 10^{-3}$ & 687 & $15^{\circ}$ & $6.4 \times 10^{24}$ & 1217 \\
B03 & $2 \times 10^{49}$ & 0.00 & 0.00 & $1.2 \times 10^{-4}$ & 0.04 & 0.00 & 16 & $3^{\circ}$ & $1.9 \times 10^{22}$ & 142 \\
B04 & $10^{48}$ & 0.17 & 0.26 & 0.52 & 0.65 & $5.1 \times 10^{-3}$ & 492 & $15^{\circ}$ & $3.6 \times 10^{23}$ & 1601 \\
B05 & $10^{50}$ & 0.30 & 0.37 & 0.60 & 0.71 & $8.3 \times 10^{-3}$ & 979 & $25^{\circ}$ & $3.6 \times 10^{25}$ & 1848 \\
B06 & $10^{49}$ & 0.19 & 0.29 & 0.54 & 0.66 & $3.7 \times 10^{-3}$ & 717 & $18^{\circ}$ & $3.4 \times 10^{24}$ & 1761 \\
B07 & $1.65 \times 10^{49}$ & 0.14 & 0.25 & 0.59 & 0.72 & $3.4 \times 10^{-3}$ & 839 & $25^{\circ}$ & $7.6 \times 10^{24}$ & 1429 \\
B08 & $1.67 \times 10^{49}$ & 0.18 & 0.25 & 0.49 & 0.60 & $4.5 \times 10^{-3}$ & 789 & $21^{\circ}$ & $5.6 \times 10^{24}$ & 1607 \\
\hline
\end{tabular}

Table 3. Fit coefficients of the lateral profiles (Eq. (12)) of the radially averaged Lorentz factor $0.4 \mathrm{~s}$ after of the cease of the energy release. Note that model B03 cannot be matched with the functional form given in Eq. (12) and $\chi^{2}$ provides a measure of the quality of the fit.

\begin{tabular}{lcccccccc}
\hline \hline Model & $a_{0}$ & $a_{1}$ & $a_{2}$ & $a_{3}$ & $a_{4}$ & $a_{5}$ & $a_{6}$ & $\chi^{2}$ \\
\hline B01 & $1.2 \times 10^{5}$ & $-5.3 \times 10^{5}$ & $-4.9 \times 10^{6}$ & $2.5 \times 10^{7}$ & $4.4 \times 10^{2}$ & $-3.1 \times 10^{3}$ & 31 & 5.3 \\
B02 & $5.9 \times 10^{2}$ & $-1.5 \times 10^{4}$ & $1.6 \times 10^{5}$ & $-2.2 \times 10^{5}$ & $1.5 \times 10^{0}$ & $-9.1 \times 10^{2}$ & 28 & 1.6 \\
B04 & $1.5 \times 10^{3}$ & $-2.2 \times 10^{4}$ & $8.3 \times 10^{4}$ & $3.4 \times 10^{5}$ & $8.1 \times 10^{0}$ & $-1.6 \times 10^{2}$ & 28 & 0.4 \\
B05 & $2.1 \times 10^{5}$ & $-1.2 \times 10^{6}$ & $-5.5 \times 10^{6}$ & $3.3 \times 10^{7}$ & $6.2 \times 10^{2}$ & $-4.4 \times 10^{3}$ & 31 & 2.8 \\
B06 & $4.5 \times 10^{4}$ & $-3.5 \times 10^{5}$ & $-1.0 \times 10^{6}$ & $1.3 \times 10^{7}$ & $1.9 \times 10^{2}$ & $-1.7 \times 10^{3}$ & 35 & 1.0 \\
B07 & $7.1 \times 10^{3}$ & $2.3 \times 10^{4}$ & $-7.1 \times 10^{5}$ & $2.7 \times 10^{6}$ & $1.8 \times 10^{1}$ & $-1.9 \times 10^{1}$ & 24 & 3.2 \\
B08 & $1.3 \times 10^{3}$ & $-1.4 \times 10^{4}$ & $9.7 \times 10^{5}$ & $-7.9 \times 10^{4}$ & $2.5 \times 10^{0}$ & $-5.8 \times 10^{1}$ & 20 & 0.9 \\
\hline
\end{tabular}

The values of the five fit coefficients $b_{i} 0.4 \mathrm{~s}$ after the shut down of the energy deposition are listed in Table 4. Simple second-order polynomials provide good fits to $\bar{e}$ in the interval $\left[0^{\circ}, 20^{\circ}\right]$.

Radial averages of the angular component of the fourvelocity $\left(\overline{\Gamma v_{\theta}}\right)$ display profiles with negative values in some regions of the body of the fireball. This is a consequence of the above mentioned rarefaction wave that results from the interaction between the external medium and the fireball. These negative velocities tend to collimate the outflow. Hence, as $\overline{\Gamma v_{\theta}}$ is a measure of the lateral expansion velocity in the comoving frame, it turns out that the part of the fireball which has average Lorentz factors $10 \lesssim \Gamma \lesssim 100$ is contracting while matter with relatively large Lorentz factors ( $\$ 100)$ expands with velocities that are considerably subsonic (Fig. 22). In this point we qualitatively agree with Kumar \& Granot (2003): The lateral expansion is slower than that assumed by the simplest homogeneous
Table 4. Fit coefficients of the lateral profiles of the radially averaged energy density (Eq. (13)) at $t=0.5 \mathrm{~s}$.

\begin{tabular}{lcccccc}
\hline \hline Model & $b_{0}$ & $b_{1}$ & $b_{2}$ & $b_{3}$ & $b_{4}$ & $\chi^{2}$ \\
\hline B01 & 19 & 4.1 & -44 & 81 & -41 & 0.003 \\
B02 & 20 & -0.26 & -37 & 88 & -53 & 0.004 \\
B03 & 19 & -4.2 & -2.6 & 22 & -15 & 0.002 \\
B04 & 18 & -0.72 & -40 & 110 & -79 & 0.010 \\
B05 & 20 & 4.3 & -33 & 44 & -13 & 0.004 \\
B06 & 19 & $8.6 \mathrm{e}$ & -73 & 140 & -82 & 0.003 \\
B07 & 19 & -0.99 & -21 & 44 & -21 & 0.005 \\
B08 & 19 & 2.5 & -45 & 94 & -53 & 0.002 \\
\hline
\end{tabular}


models (which consider expansion velocities close to the sound speed or even larger). We also point out that the absolute values of $\overline{\Gamma v_{\theta}}$ tend to decrease with time.

An upper bound for the duration of a GRB event might be set by the time interval between the moments when the forward and the rear ends of the expanding fireball reach transparency at large radii. This time difference is linked to the difference in speed between the forward and rear ends of the fireball in the radial direction. It turns out that the velocity of propagation of the forward edge corresponds to that of the terminal shock of the wind or the head of the jet in our models. This velocity is determined by the amount of mass swept up by the bow shock of the jet. Since the halo density in models of type-B is low, the propagation speed of the fireball is ultrarelativistic (Table 1). On the other hand, the velocity of the rear part of the wind is set by the speed of the contact discontinuity that separates the fireball from the external medium after the energy release has stopped. Behind this wave, matter fills the wake of the fireball, i.e., the ultrarelativistic wind structure is lost. The contact discontinuity propagates at a speed very close to the speed of light because the relativistic wind is very rarefied. In addition, the speed of this wave is not constant, because the plasma in the fireball experiences ongoing acceleration during the transformation of its internal energy into kinetic energy. In order estimate this velocity we have approximated the hydrodynamic states at the rear, radial edge of the fireball by two constant states corresponding to the fireball (right) and to the external medium (left). The two states are (in cgs units): $\left(p_{\mathrm{L}}, \rho_{\mathrm{L}}, \Gamma_{\mathrm{L}}\right) \approx$ $\left(10^{14}, 4 \times 10^{-4}, 2\right)$ and $\left(p_{\mathrm{R}}, \rho_{\mathrm{R}}, \Gamma_{\mathrm{R}}\right) \approx\left(6 \times 10^{14}, 10^{-7}, \Gamma_{\mathrm{R}}\right)$. The generic solution of the Riemann problem (see above) consists of a contact discontinuity flanked by two rarefaction waves (one advancing into the fireball and the other one extending into the external medium) where the fluid progressively accelerates from $\Gamma_{\mathrm{L}}$ to $\Gamma_{\mathrm{R}}$. We have computed the exact solution of the Riemann problem set by this series of states as a function of $\Gamma_{R}$ with $80 \leq \Gamma_{R} \leq 220$, which are typical values of the Lorentz factor near the rear edge of the fireball as it accelerates after the energy deposition is stopped. The Lorentz factor associated with the contact discontinuity $\left(\Gamma_{\mathrm{CD}}\right)$ is a monotonic function of $\Gamma_{\mathrm{R}}$ that can be fitted by the linear relation

$\Gamma_{\mathrm{CD}} \approx 6.42 \times 10^{-2} \Gamma_{\mathrm{R}}+12.60$,

i.e., $\Gamma_{\mathrm{CD}} \leq \Gamma_{\mathrm{R}}$ for ultrarelativistic fireballs.

$\Gamma_{\mathrm{R}}$ is, in turn, smaller than the Lorentz factor associated with the leading radial edge of the fireball during the acceleration phase of the outflow, which leads to a progressive stretching of the fireball in the radial direction. A possible important consequence of this stretching is that the duration of the GRB event might be considerably longer than the time interval of the activity of the central engine. If the fireball stretches radially on its way to the optically thin regime, the on-time of the energy source would not necessary be a suitable measure of the GRB duration. In order to derive an order of magnitude estimate of the stretching effect it is necessary to extrapolate from our computed evolution to much later times. For this purpose we have calculated from our hydrodynamic models the speed of the fireball tail and compared it with the analytic estimate of Eq. (14). It turns out that the analytic result agrees reasonably well with the numerical computation. The contact discontinuity, which roughly corresponds to the tail of the fireball, moves with an average velocity of $v_{\mathrm{CD}}=0.99_{-0.04}^{+0.009}$. Hence, if the burst is produced after the fireball has traveled a distance $R$, an upper bound to its duration will approximately be given by

$\Delta t_{\mathrm{b}} \lesssim\left(R-\frac{v_{\mathrm{CD}}}{v_{\mathrm{p}}}\left(R-t_{\mathrm{sa}} c\right)\right) c^{-1}$,

where $v_{\mathrm{p}}$ is the propagation velocity of the fireball in the radial direction and $t_{\mathrm{sa}}=0.1 \mathrm{~s}$ is the time of source activity.

Taking a typical distance $R=10^{13} \mathrm{~cm}$, Eq. (15) yields a maximum burst duration of $\Delta t_{\mathrm{b}} \simeq 4.3_{-3.0}^{+10.3} \mathrm{~s}$, which is considerably longer than the time interval of source activity $\left(t_{\mathrm{sa}}=0.1 \mathrm{~s}\right)$. It is emphasized that Eq. (15) and the arguments given in this context do not allow one to make quantitatively meaningful predictions for observable GRB durations. The estimate of Eq. (15) assumes that no deceleration of the fireball head occurs by the accumulation of matter that is swept up from the ambient medium until the fireball reaches the distance $R$. A detailed model of the long-term evolution and structure of the fireball also requires a realistic, time-dependent model for the torus accretion and the associated energy release (see the subsequent discussion of models B07 and B08). Moreover, the velocities of the head and the tail of the fireball cannot be extracted to very high accuracy from our relativistic hydrodynamics results (mainly because of the limited resolution at large radial distances), but are nevertheless used to extrapolate the simulations by several orders of magnitude in radius. The velocities also do not need to be decisive for the actual duration of an observable GRB, in particular, because the tail velocity was deduced from the motion of the rear contact discontinuity of the fireball, which might not be the backward boundary of highly relativistic matter with $\Gamma>100$ that forms the $\gamma$-ray emitting part of the GRB fireball. Equation (15) is mainly meant to manifest the fact that the GRB duration, in particular for short bursts, is not necessary given by the on-time of the energy source, but can be significantly longer due to the discussed fireball stretching. Indeed, the stretching may account for the major part of the GRB duration. The numerical estimates obtained from Eq. (15) at best are rough upper limits to this effect. In contrast, in the collapsar model for long bursts, for example, the accretion time and thus the period of source activity clearly dominates the observed GRB duration.

\subsection{Models B02 to B06}

This series of models has in common with B01 that the source activity time is limited to $0.1 \mathrm{~s}$. In this section we shall discuss which are the similarities and differences of their evolution after the end of the energy release relative to model B01. In Fig. 25 the fluid Lorentz factor of all type-B models is displayed at the end of our computed evolution $(0.5 \mathrm{~s})$. The high Lorentz factors suggest that all models, except B03, may be suitable for producing cosmic GRBs (at least they fulfill the canonical requirement of $\Gamma \gtrsim 100$ ). All models have outflow opening angles which are smaller than the opening angle of the deposition region (see also Table 2), confirming once again that the opening angle of the outflow is set by its interaction 
with the side walls of the torus. The diversity of opening angles (from $3^{\circ}$ to $25^{\circ}$ ) is caused by the differences in the energy that is released around the $\mathrm{BH}$ per unit of time and unit of volume, which also depends on the angular width of the deposition region. The trend is that models with a larger $\dot{E} / V_{\text {dep }}$ develop larger outflow opening angles. Model B03 is special only by the fact that due to the large opening angle of the deposition cone and a modest total rate of energy release, most of the energy is deposited in the densest part of the torus and hence can only trigger a moderately relativistic outflow. In case of models B02 and B04 the final opening angle of the ultrarelativistic outflow, might be smaller than at $0.5 \mathrm{~s}$ because both models show substantially negative values of $\overline{\Gamma v_{\theta}}(\approx-0.5 c$; Fig. 23$)$.

Stretching of the fireballs in the radial direction should occur in all models discussed in this section, and is indeed found up to $0.5 \mathrm{~s}$. The radial extension increases similarly in all models. In agreement with our numerical results and, according to our estimate of Eq. (14), the small differences result from somewhat different velocities of the tail of the fireballs (the forward radial front of the fireballs moves in all the cases except B03 at $v_{\mathrm{p}} \simeq c$ ).

The maximum Lorentz factor found within the fireball at $t=0.5 \mathrm{~s}$ varies substantially from model to model and lies in the range from $\sim 16$ to $\sim 1000$ (Table 2 ), following the trend that a larger $\dot{E} / V_{\text {dep }}$ leads to a larger maximum Lorentz factor. Figure 24 shows that soon after the end of energy release the total (internal plus kinetic) isotropic equivalent energy of the fireball core reaches a maximum and saturates, while there is an ongoing transformation of internal into kinetic energy (which is constantly rising). During this conversion of energies the Lorentz factors within the fireball grow. They have not saturated until the end of the simulations.

A comparison of the estimated terminal Lorentz factors $\Gamma_{\infty}$ of the different models (Table 2) reveals that models B01, B04, B05 and B06 are somewhat more efficient than models B02 and B03, the main reason being the larger opening angle of the energy deposition cone in the latter two cases in combination with a smaller energy deposition rate per volume. This causes an increase of the baryon loading of the fireball relative to the energy stored in the pair-photon plasma and is a crucial difference between models B03 and, e.g., B04. The latter develops an energetic ultrarelativistic jet although the energy deposition per solid angle is smaller by a factor of $\sim 10$ than in B03. In model B03 the estimate of the asymptotic Lorentz factor is not meaningful because the model develops a collimated jet with only a very narrow and light relativistic core (where $\Gamma \gtrsim 10$; Fig. 23). As the estimate $\Gamma_{\infty}$ only considers the mass of the outflow with $\Gamma>10$, the amount of mass that fulfills this criterion in model B03 is dynamically negligible compared to the mass of the whole outflow, which would have to be used for calculating the asymptotic value of $\Gamma$ in this case.

All models (except B03) display a similar structure of the radially averaged energy density (Fig. 23). Close to the symmetry axis and up to a few degrees off axis it is nearly constant. At larger angles $\theta$ a hump appears where the energy density is about a factor of three higher than near the axis. From this local maximum the energy density declines gently towards the lateral edges of the fireball where it starts rising again due to the
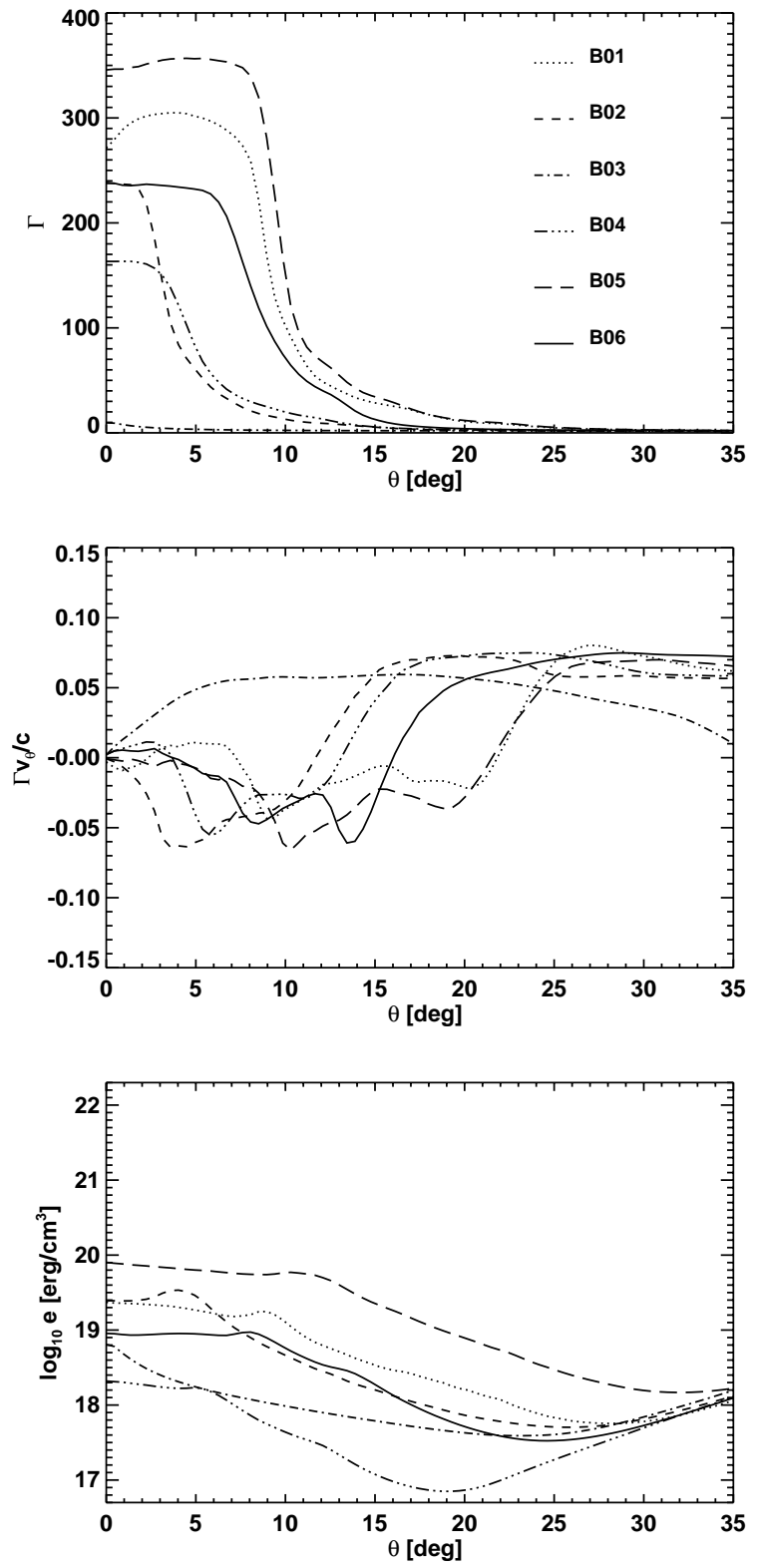

Fig. 23. Radially averaged values of the Lorentz factor (top panel), angular component of the four-velocity (intermediate panel) and energy density (bottom panel) as functions of the polar angle after $0.5 \mathrm{~s}$ of evolution. The different lines correspond to different models from B01 to B06.

interaction with the external medium. The energy hump within the fireball coincides with a region of large compression of the fluid where the comoving lateral velocity reaches a local minimum that, in some models (e.g., in B02, B04 and B05), is an absolute minimum; (Fig. 23, middle panel).

The isotropic equivalent total energy as a function of polar angle $\theta$ for matter with Lorentz factors larger than 50 (Fig. 24) consists of two parts: a central core of $4^{\circ}$ (model B02) to $10^{\circ}$ (model B01) where it is nearly uniform and maximal, surrounded by an extended layer where it drops roughly like $\theta^{-2}$. The generation of the energy profile of the burst seems neither to depend on the width of the deposition region (it is similar for a wide range of $\theta_{0}$ values) nor on the absolute value of the 


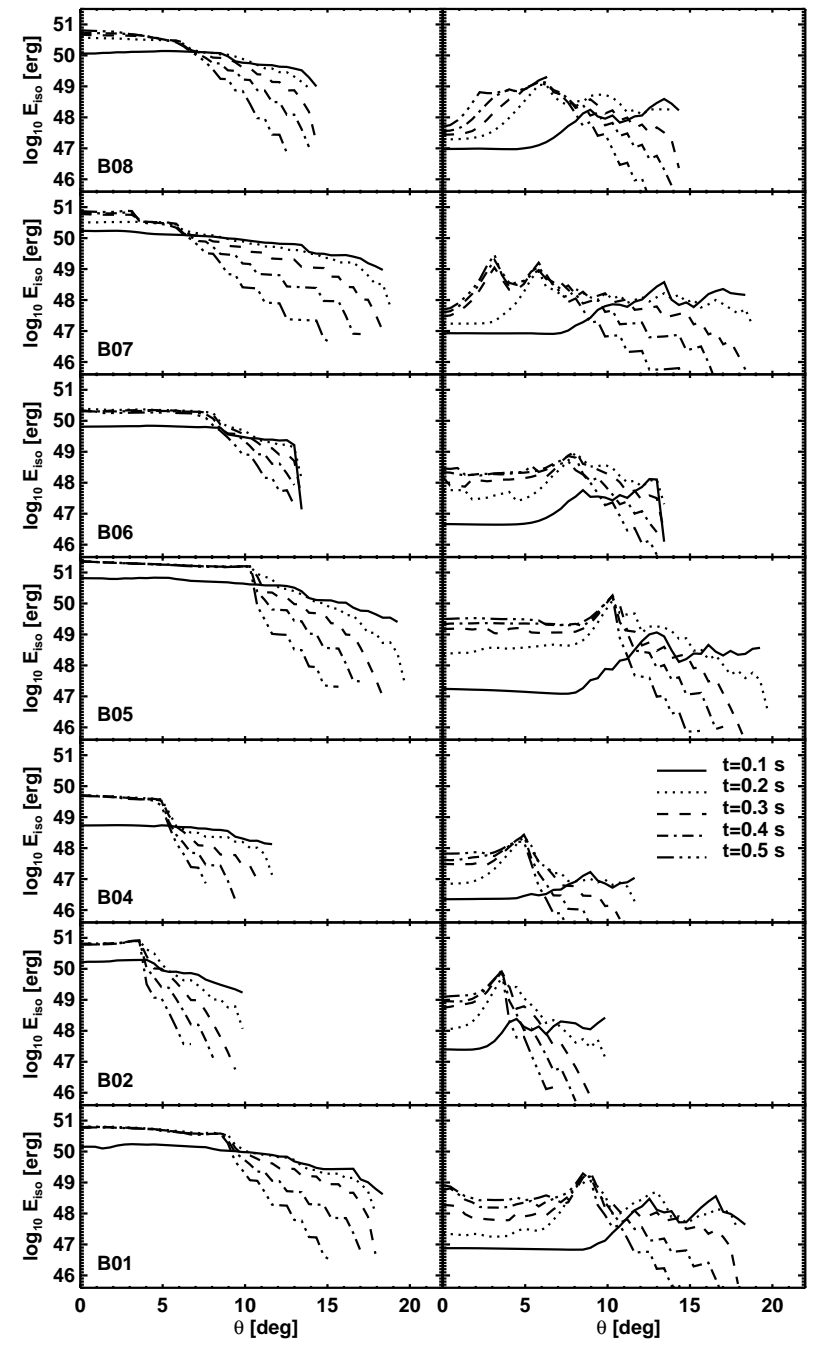

Fig. 24. Isotropic equivalent total (left) and kinetic (right) energies of matter with $\Gamma>100$ for all B-type models at different evolutionary times. Model B03 is omitted in this figure because it does not develop an outflow with $\Gamma>100$. Note that the central plateau of the isotropic equivalent total energy increases substantially between $0.1 \mathrm{~s}$ and $0.2 \mathrm{~s}$ when the source is already off. This is due to the continuous acceleration of matter to Lorentz factors above 100 as a result of the conversion of internal to kinetic energy in the fireball (even after the shut-off of the energy release).

energy deposition rate (or its time dependence). Nevertheless, we are not in a condition yet to claim that this is a universal profile, because it might be linked to the particular spatial dependence of the assumed energy deposition law or to the properties of the BH-torus system. Future simulations will have to investigate these aspects.

\subsubsection{Models B07 and B08}

In these models, the energy is released with a time-variable deposition rate (Eq. (10)) and continues for longer that $100 \mathrm{~ms}$. Note that due to the assumed power-law decay the energy deposition rate is still at a level of $3.5 \times 10^{48} \mathrm{erg} \mathrm{s}^{-1}$ after $500 \mathrm{~ms}$. This is the reason why the high-Lorentz factor outflow that has formed an almost conical fireball does not detach from the energy deposition region and, thus, the axial funnel of low density and high Lorentz factor stays open until the end of the computed evolution (Figs. 25, 26, 29, 28). This axial spine decreases in width with time and, if we continued to follow the evolution of B07 or B08 for a longer period, the energy supply at some later time would not be sufficient any longer to keep the funnel open.

The structure of the fireball is non-uniform (Figs. 29, 25) and its morphology does not differ substantially from that of model B01 (except for the long trailing tail which results from the continuous energy deposition). The plateau of the maximal, radially averaged Lorentz factor is more narrow $\left(\approx 2^{\circ}\right.$ in both model B07 and B08) than in model B01 (Fig. 27), because of the progressive contraction of the fireball fluid (which is larger in model B07 than in B08; Fig. 27). Furthermore, the radially averaged maximum Lorentz factor after $0.5 \mathrm{~s}$ is larger for these two models (with values above 375 ) than for B01. However, the absolute maximum Lorentz factor $\left(\Gamma_{\max }\right)$ at the end of the simulations corresponds to model B01 (Table 2). The reason for these differences is that B01 is more efficient than B07 or B08 in using the released energy to accelerate matter to Lorentz factors above 100 (see the values of $E_{\Gamma>100} / E_{\mathrm{d}}$ in Table 2).

The estimates for the asymptotic Lorentz factor $\Gamma_{\infty}$ for models B07 and B08 in Table 2 may not be meaningful because energy is released even beyond the simulated epoch of evolution.

The time variability of the energy deposition rate of model B08 produces a wrinkled boundary of the fireball with a modulation period of $10 \mathrm{~ms}$ as the energy release (Fig. 28, left panel). A similar wrinkled surface occurs in model B05 (Fig. 16), but different from model B08 the modulation of the density in the surface is not exactly periodic, and the values of the density in the fireball core up to $\sim 2 \times 10^{9} \mathrm{~cm}$ are much higher $\left(\sim 10^{-2} \mathrm{~g} \mathrm{~cm}^{-3}\right.$ very near the axis; Fig. 16). After $0.25 \mathrm{~s}$ models B07 and B08 display a fairly similar structure. This is, to a large extent, due to the grid coarsening in radial direction. Beyond a distance of $\approx 10^{10} \mathrm{~cm}$ the grid spacing exceeds 10 light-milliseconds and, therefore, most of the variability on scales less than $\approx 15 \mathrm{~ms}$ is damped or erased. For this reason, we cannot draw firm conclusions on the short-time variability from our models, and in particular we cannot answer the question how it depends on the intrinsic variability of the source or the interaction with the external medium.

The radially averaged energy density of models B01, B07 and B08 varies somewhat differently with the polar angle (Fig. 27). The central, most energetic core of the fireball of models B07 and B08 is more narrow. Model B08 does not possess a clear plateau around the symmetry axis and also has, on average, a smaller energy content compared to the other two models. The kinetic energy in the central core of the ultrarelativistic outflow is smaller than in the surrounding layer in all B-models at $t=0.5 \mathrm{~s}$ (Fig. 30) because the higher Lorentz factors in the cores are compensated by larger densities outside. The efficiency for converting thermal to kinetic energy until $\sim 0.5 \mathrm{~s}$ is $\sim 25 \%$ smaller in case of model B07 than in model B01 or B08 (Table 2). However, in all three cases and all other B-models the kinetic energy in the fireball relative to the total energy of matter with $\Gamma>100$ is still at most a few 


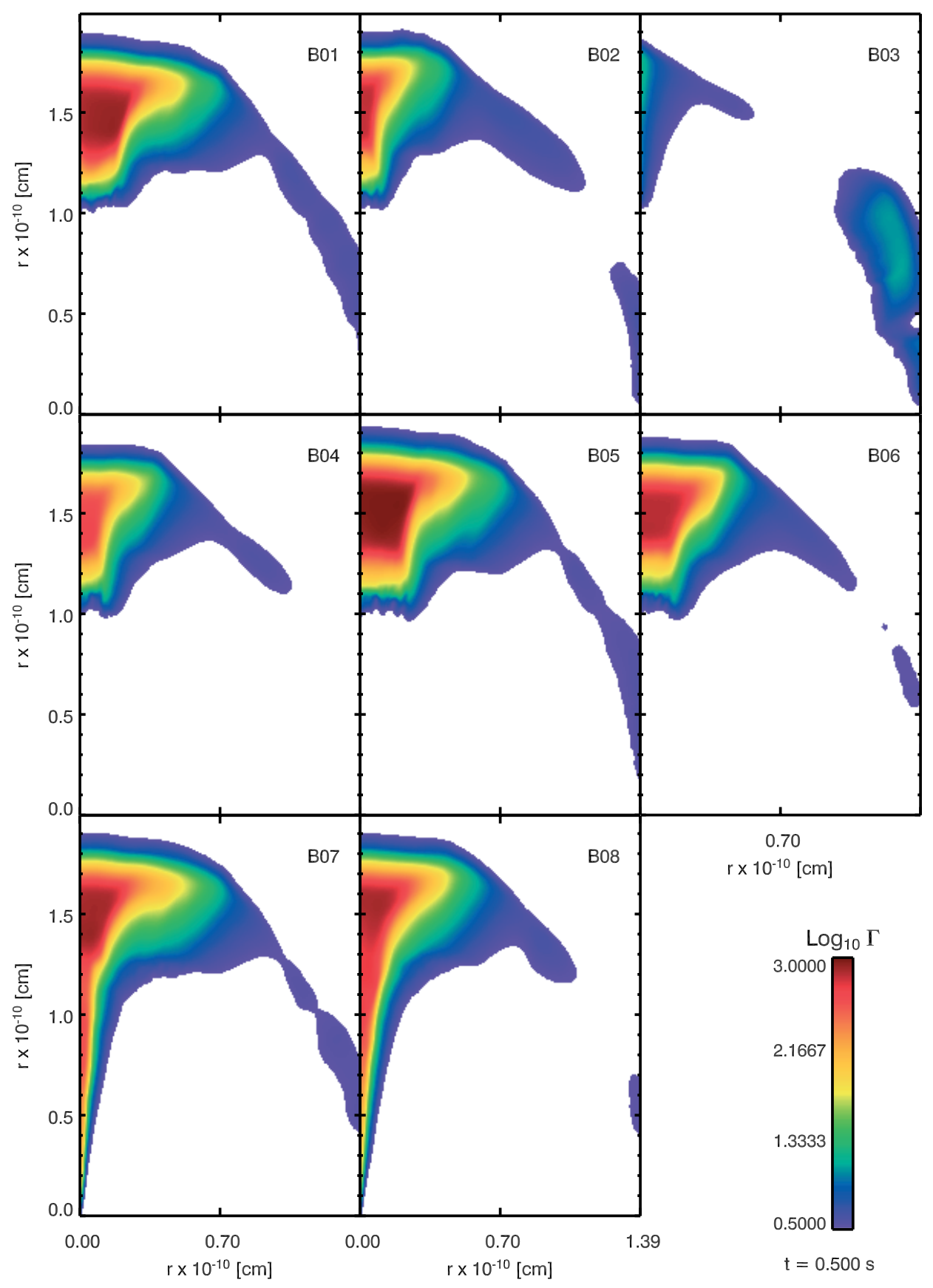

Fig. 25. Logarithm of the Lorentz factor of the type-B models after $0.5 \mathrm{~s}$ of evolution time. The color scale is limited in order to enhance the details of the outflow region.

per cent at $t=0.5 \mathrm{~s}$ (Table 2), i.e., there is ample room for further fluid acceleration. Figure 30 shows that only model B03 is an exception, as it has essentially reached its terminal state. Figure 30 also shows that the contribution to the kinetic energy of the parts of the outflow having bulk Lorentz factors larger than 50 is small while most of the kinetic energy is transported by fluid with Lorentz factors below 10. It is also evident that the fraction of kinetic to internal energy at $0.5 \mathrm{~s}$ is closer to unity for matter with low Lorentz factors farther off axis, i.e., this material will not reach much higher asymptotic velocities during its adiabatic expansion. The ultrarelativistic core, however, can be expected to continue accelerating, when its internal energy is converted to kinetic energy.

The evolution of the ratio of kinetic energy to total (internal plus kinetic) energy in the outflow of the prototype model B01 is shown in Fig. 31. In this figure the kinetic and total energies of the relativistic outflow are computed as the integral values for all cells with $\Gamma>\Gamma_{\min }$ for different lower limits $\Gamma_{\min }$ of the Lorentz factor. One can notice a slight, non-monotonic increase of the fraction of kinetic energy with time. Several conclusions can be drawn from Fig. 31. First, if the rate of increase can be extrapolated until the time when transparency sets in, it must be 


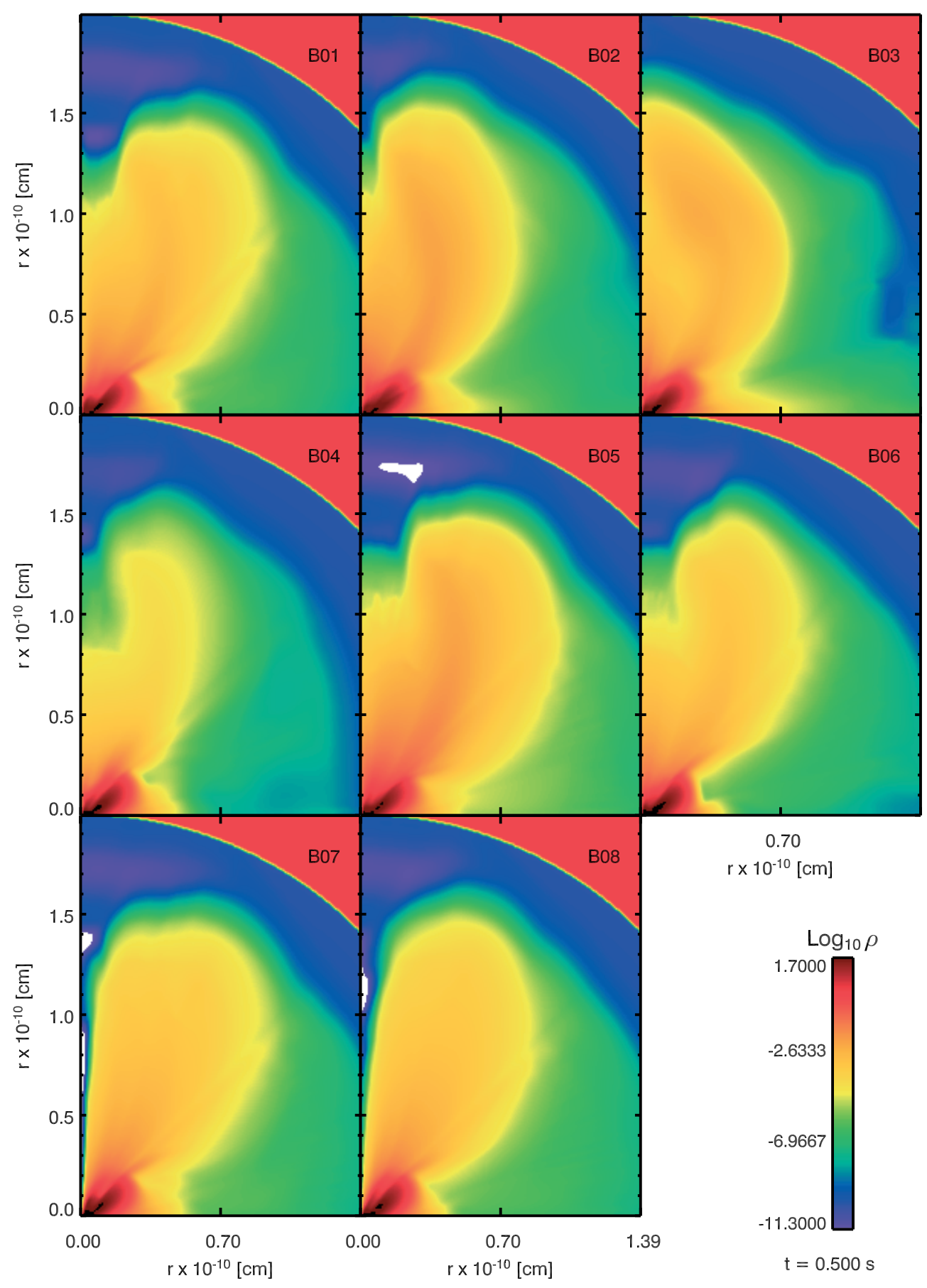

Fig. 26. Logarithm of the rest-mass density $\left(\right.$ in $\mathrm{g} \mathrm{cm}^{-3}$ ) of the B-models after $0.5 \mathrm{~s}$ of evolution time. The color scale is limited in order to enhance the details of the outflow region.

expected that most of the internal energy will be converted to kinetic energy. Second, after $0.5 \mathrm{~s}$ of evolution about half of the kinetic energy of the outflow is in fluid with $\Gamma \geq 10$. However, it is impossible to reliably predict the final distribution of kinetic energy versus Lorentz factor (after, e.g., $\sim 1000 \mathrm{~s}$ ), because the trends shown for fluid elements with different values of $\Gamma_{\text {min }}$ are slightly different and, in some cases it is hardly possible to deduce an accurate number for the net increase of the ratio of kinetic to total energy (e.g., in case of $\Gamma_{\min } \geq 100$; Fig. 31).

\section{Summary and conclusions}

We have performed general relativistic, axisymmetric hydrodynamic simulations to study the creation and evolution of relativistic outflow in response to the deposition of thermal energy above the poles of a black hole-accretion torus system. The latter is considered to be a necessary condition for obtaining baryon-poor fireballs, in particular if the energy is provided by neutrino-antineutrino annihilation. Above the poles 

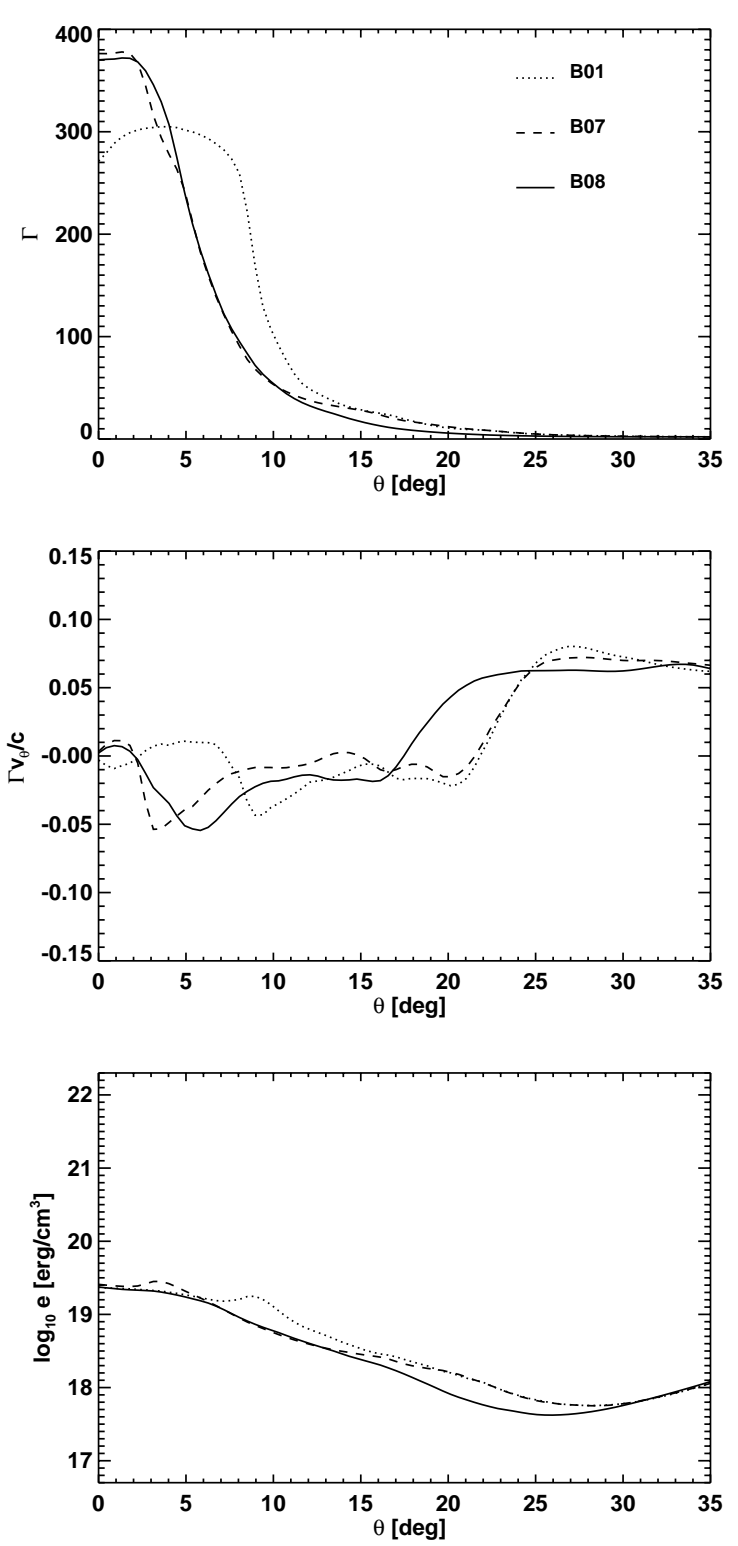

Fig. 27. Radially averaged values of the Lorentz factor (top panel), angular component of the four-velocity (intermediate panel) and energy density (bottom panel) as functions of the polar angle after $0.5 \mathrm{~s}$ of evolution. The different lines correspond to models B01, B07 and B08, respectively.

of the black hole a favorable environment is encountered for the creation of an ultrarelativistic $\mathrm{e}^{ \pm}$-pair-photon plasma by the mentioned process, different from the situation in case of neutron stars, where neutrino energy deposition in the surface-near layers produces a nonrelativistic baryonic wind (Duncan et al. 1986; Woosley 1993; Qian \& Woosley 1996; Thompson et al. 2001).

Being guided by numerical models of Ruffert \& Janka (1999) and Janka et al. (1999), the BH-torus systems for our studies were set up to represent the remnants of NS+NS or $\mathrm{NS}+\mathrm{BH}$ mergers. We chose Schwarzschild BHs of $2.44 M_{\odot}$ or $3 M_{\odot}$, being girded by a thick torus of $0.13 M_{\odot}$ or $0.17 M_{\odot}$, respectively. The accretion torus was constructed either by relaxation of an initial toroidal cloud of matter or by creating a near-equilibrium configuration with the technique of Font \& Daigne (2002). In the first case the BH-torus system is surrounded by a fairly dense $\left(\sim 100 \mathrm{~g} \mathrm{~cm}^{-3}\right)$ gas halo with a radius of about $10^{9} \mathrm{~cm}$ (containing a mass of roughly $10^{-4} M_{\odot}$ ), e.g., considered to be the consequence of a neutrino-driven wind from a transient, metastable NS which underwent collapse to a $\mathrm{BH}$-torus system only after some time delay. In the second case the density of the surrounding gas is much lower and decreases radially approximately as $r^{-3.4}$. Correspondingly, we discriminate between high-density type-A models and low-density type-B models. Both are considered as possible results of a violent, preceding merger history. Thermal energy was deposited within a cone with chosen opening angle above the poles of the black hole, starting at the inner grid boundary at $4 G M_{\mathrm{BH}} / c^{2}$ or $2 G M_{\mathrm{BH}} / c^{2}$ for type-A or type-B models, respectively. Cases with constant energy deposition rate were studied or with a burst-like initial phase, followed by a long-time, gradual decay. The energy deposition rate was assumed to depend only on the vertical height $z$ measured along the rotation axis and to drop like $z^{-5}$. This prescription is an approximate representation of deposition maps obtained by detailed calculations of neutrinoantineutrino annihilation in the vicinity of the BH-torus system (Ruffert \& Janka 1999; Janka et al. 1999). Disregarding the modest lateral bending of the computed surfaces of constant $v \bar{v}$-energy deposition rate turned out not to be essential for the discussion in this work, but the deviation of these surfaces from spheres had important consequences.

We calculated sequences of type-A and type-B models, systematically varying the total rate of energy deposition $(\dot{E})$ while keeping the lateral width of the deposition region unchanged. Alternatively, we changed the opening angle of the energydeposition cone for a fixed integral rate or for a fixed energy deposition rate per unit of volume $\left(\dot{E} / V_{\text {dep }}\right)$, respectively. In summary, our studies revealed the following dynamical behavior of the evolving outflow:

- Relativistic outflow develops in form of narrow, knotty jets or wide, ultrarelativistic winds or jets. The generation of relativistic outflow requires a high energy deposition rate or low environmental density. In case of the high-density type-A models the energy deposition rate has to exceed a certain threshold value (around $10^{49} \mathrm{erg} \mathrm{s}^{-1}$ ) in order to produce any outflow. Slightly above this threshold an inhomogeneous jet is formed rather than an ultrarelativistic wind.

- In the case of type-A models the collimation of the outflow is mainly determined by the interaction with the highdensity ambient medium. The opening angle of the polar outflow increases with $\dot{E} / V_{\text {dep }}$ and varies from a few degrees for the low-density (baryon-poor) jets that develop just above the ejection threshold to more than $25^{\circ}$ for the smooth, low-density, wide-angle winds in case of the highest considered $\dot{E} / V_{\text {dep }}$. The interaction between outflow and ambient medium resembles the hydrodynamic collimation mechanism discussed by Levinson \& Eichler (2000), and the unshocked part of the baryon-poor outflow can be well fitted by power laws with indices close to the ones predicted by the analytic model of these authors. Because of the large mass that is swept up, the 


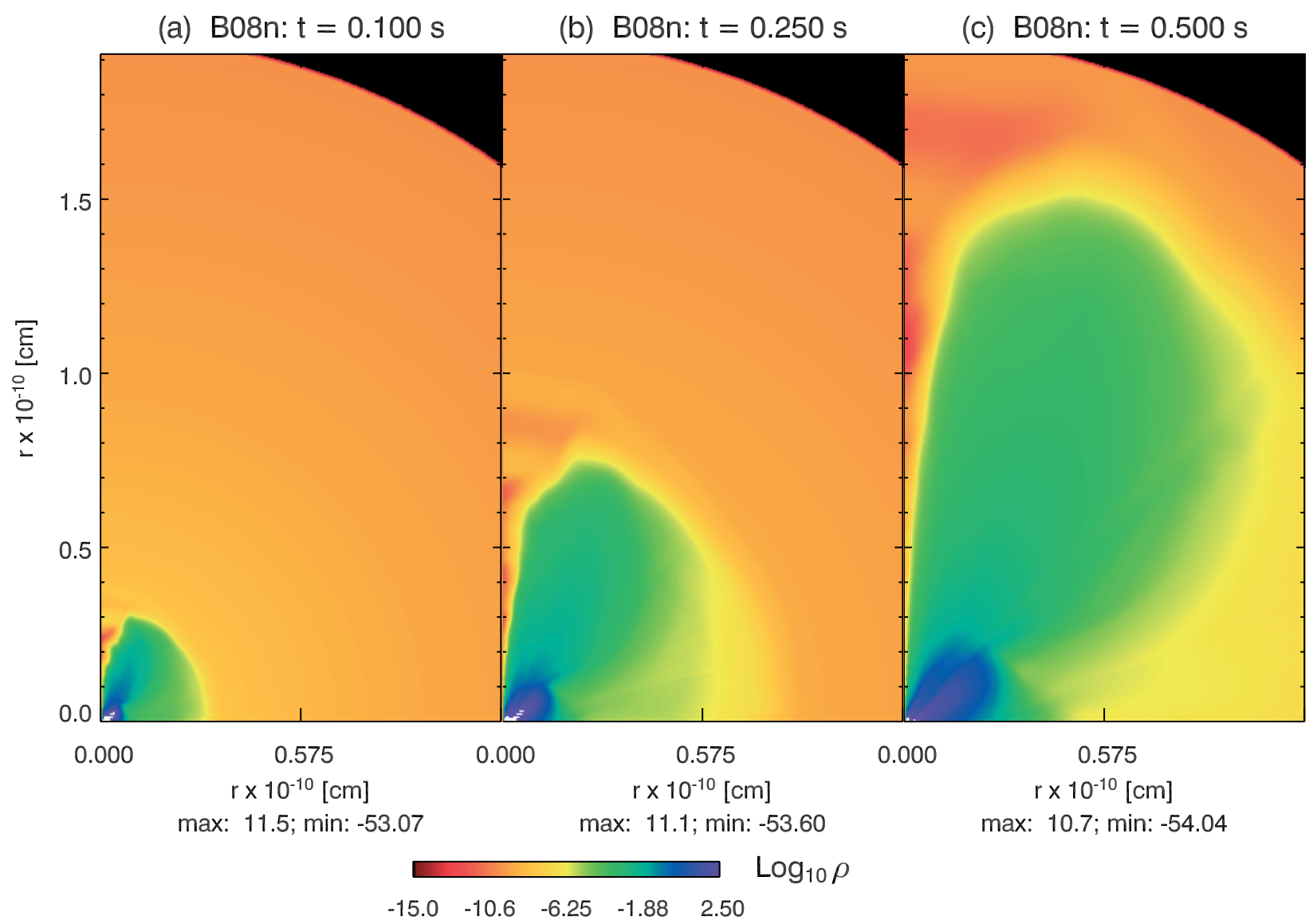

Fig. 28. Evolution of the logarithm of the rest-mass density $\left(\mathrm{g} \mathrm{cm}^{-3}\right)$ of model B08 after $100 \mathrm{~ms}$. Time is measured from the moment when the energy deposition was started.

propagation speed of the shocked, forward part of the outflow becomes only mildly relativistic in all models, not allowing for favorable conditions to produce GRB emission. Instead, a low-luminosity (at most $\sim 10^{43} \mathrm{erg} \mathrm{s}^{-1}$ ) soft UV-flash at a temperature of $\sim 5 \times 10^{4} \mathrm{~K}$ with a duration of $\sim 1000 \mathrm{~s}$ can be expected as the observable signature of merger events surrounded by high-density halos. Due to their small luminosity, only nearby events might be detected.

- In the case of type-B models all employed energy deposition rates (above $\sim 10^{49} \mathrm{erg} \mathrm{s}^{-1}$ ) led to non-uniform, wideangle, radially and laterally structured ultrarelativistic jets provided the rate of energy deposition per unit of volume was sufficiently high. The propagation velocities are found to be very close to the speed of light, corresponding to Lorentz factors of several hundred, also in the forward, shocked part of the flow. This suggests favorable conditions for the production of GRBs. The simple power-law fits of the analytic model of Levinson \& Eichler (2000) do not work well. The latter authors assumed that a baryonpoor jet is confined by the ram pressure of a baryonic (nonrelativistic) wind which originates from a toroidal ring of negligible thickness. They further assumed that the baryonrich wind collides with the relativistic jet and gets deflected into a very thin, shocked layer that envelopes the relativistic flow. Our hydrodynamic simulations do not support this picture. We found that the collimation of the baryon-poor flow in type-B models is caused by the interaction of the accelerating fluid with the dense accretion torus, whose vertical extension cannot be ignored. This interaction produces a thick shell of gas which surrounds the ultrarelativistic core and which shows large inhomogeneities due to Kelvin-Helmholtz instabilities. The half-opening angle of the ultrarelativistic flow is set within the first millisecond of its expansion on a distance that corresponds to the vertical thickness of the accretion torus. It is determined by the curvature of the torus walls around the poles of the $\mathrm{BH}$ and was found to be around $20^{\circ}$ in the computed type-B models. - Type-A and type-B models also showed a different behavior after the deposition of energy was switched off or had decayed. In case of a high-density halo around the BH-torus system (type-A) the conical, relativistic wind structure is destroyed within a few source activity timescales $\left(t_{\mathrm{sa}}\right)$, because the tail of the outflow moves into a cleared funnel and is much faster. Therefore, it catches up with the leading edge of the flow, which gets decelerated by swept-up matter. In contrast, in type-B models with their steeply decreasing halo density, the front part of the outflow is steadily accelerated while its rear end moves somewhat slower because of the lack of support by further strong energy release at the origin. Therefore the fireball cone is preserved instead of being destroyed. However, preservation is a 


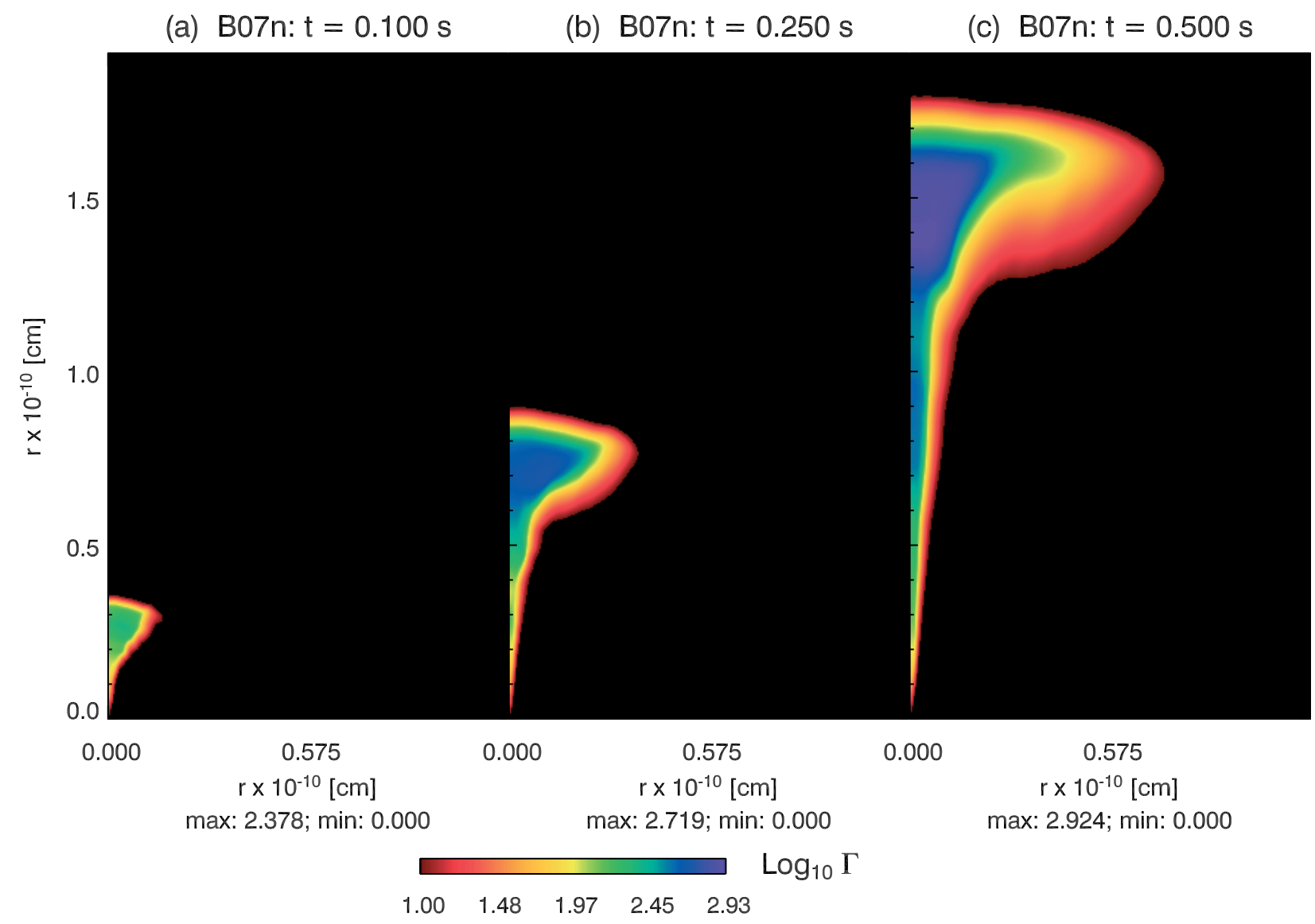

Fig. 29. Evolution of the logarithm of the Lorentz factor of model B07 after $100 \mathrm{~ms}$. Time is measured from the moment when the energy deposition was started.

necessary condition for GRB production. The fireball can in fact be radially stretched before it reaches optically thin conditions. Assuming that the GRB duration, $\Delta t_{\mathrm{b}}$, is defined by the time difference between the front and rear ends of the fireball reaching the "transparency radius", e.g., $10^{13} \mathrm{~cm}$, we can estimate, by extrapolating the results of our computed models, a burst duration $\Delta t_{\mathrm{b}}$ that might be significantly longer than the on-time $(\sim 0.1 \mathrm{~s})$ of the source: $\Delta t_{\mathrm{b}} \cong 4.3_{-3.0}^{+10.3} \mathrm{~s}$. Note that this stretching can be the dominant contribution to $\Delta t_{\mathrm{b}}$ in case of source activity times of significantly less than one second as expected for the accretion timescale of post-merger disks. The latter timescale is expected to vary with the torus mass. It is set by the viscous transport within the compact accretion torus, in contrast to collapsars where the accretion disk is fed by an external reservoir of several solar masses of stellar matter and the accretion timescale is therefore determined by the collapse timescale of the massive, rotating star. Even if the accretion phase of the remnant $\mathrm{BHs}$ of $\mathrm{NS}+\mathrm{NS}$ or $\mathrm{NS}+\mathrm{BH}$ mergers lasts only fractions of a second, our simulations suggest that such events can account for the measured durations of short GRBs. Of course, our estimation of $\Delta t_{\mathrm{b}}$ can only be considered as an exercise for demonstrating a fundamental possibility. It has no predictive power for GRB durations, because we need to extrapolate our hydrodynamic results over several orders of magnitude in radius.
This is rather uncertain due to difficulties in extracting accurate values for the fluid velocities from the computations in case of very high Lorentz factors and due to the geometrical dilution of the spatial grid resolution with increasing radial distance. Extrapolation of our results from about $0.5 \mathrm{~s}$ ( $\sim 0.4 \mathrm{~s}$ ) after the onset (shutdown) of the energy release to more than $300 \mathrm{~s}$ later also ignores how the fireball properties continue to change and how the long-time propagation and expansion of the fireball may depend on the structure of the ambient medium of the merger site. Moreover, the GRB emission might be shorter than our estimate if it is produced in a region that is smaller than the whole fireball. Nonthermal emission of radiation requires the dominant energy of the flow to be kinetic energy of relativistic baryons but not internal energy. Unfortunately our simulations had to be stopped before definite statements about the terminal fireball structure and the final ratio of its kinetic to internal energy were possible.

- The total energy (internal plus kinetic) of the relativistic fireball scales roughly linearly with the deposited energy and saturates after the energy release has ended. A burstlike, short initial phase of energy deposition, followed by a long-time, gradual decay turned out to channel somewhat less energy into the relativistic outflow and to be slightly less efficient in converting internal energy to kinetic energy than a constant rate of energy release with a sudden end. In 


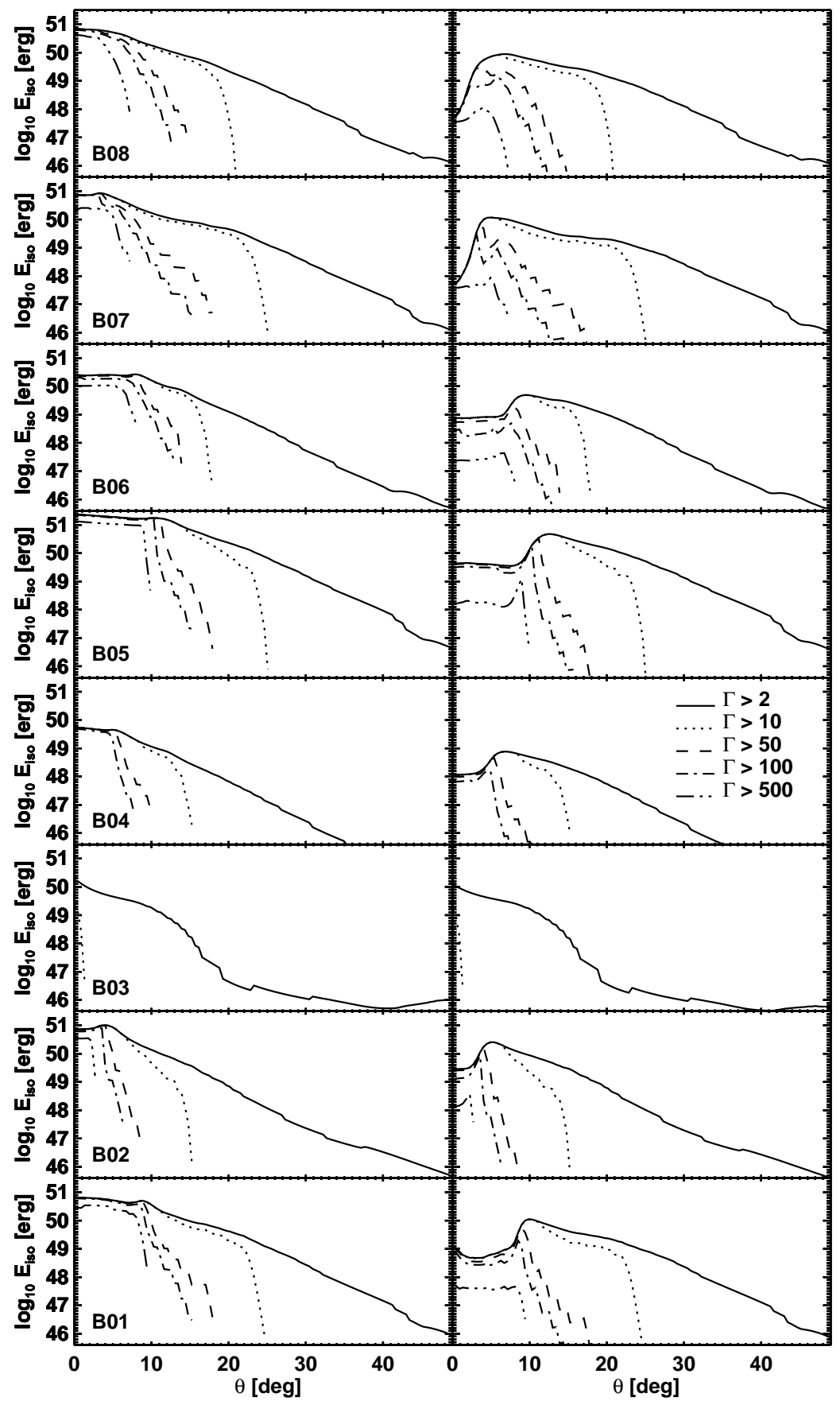

Fig. 30. Isotropic equivalent total energy (left panels) and isotropic equivalent kinetic energy (right panels) corresponding to outflows with Lorentz factors above certain thresholds at $500 \mathrm{~ms}$ of computed evolution. Resuls for different thresholds are plotted with different line styles. Note that in the panels corresponding to model B03 there are no lines for Lorentz factor thresholds above 50, because there is no outflow with such a large Lorentz factor.

both cases a minor part (a few per cent at most) of the energy was in form of kinetic energy after $0.5 \mathrm{~s}$ of computed evolution, with the tendency to continue rising.

- The relativistic flow in type-B models reveals a highly inhomogeneous structure in both the radial and lateral directions. A small negative radial gradient of the radiationdominated pressure corresponds to a radial increase of the Lorentz factor also in the shocked region of the flow, where the average behavior of different quantities can roughly be described by power laws of the radius (though different from those of the analytic model of Levinson \& Eichler 2000). While pressure and Lorentz factor reveal only moderate variations (factors of a few), the density shows huge fluctuations up to three orders of magnitude. These inhomogeneities are a relic of Kelvin-Helmholtz instabilities which are triggered by the interaction of the fireball with the torus medium. In lateral direction the structure cannot be fitted by simple top-hat, power-law or Gaussian profiles but can posses off-axis maxima of the Lorentz factor. This feature results from a genuinely relativistic effect that 

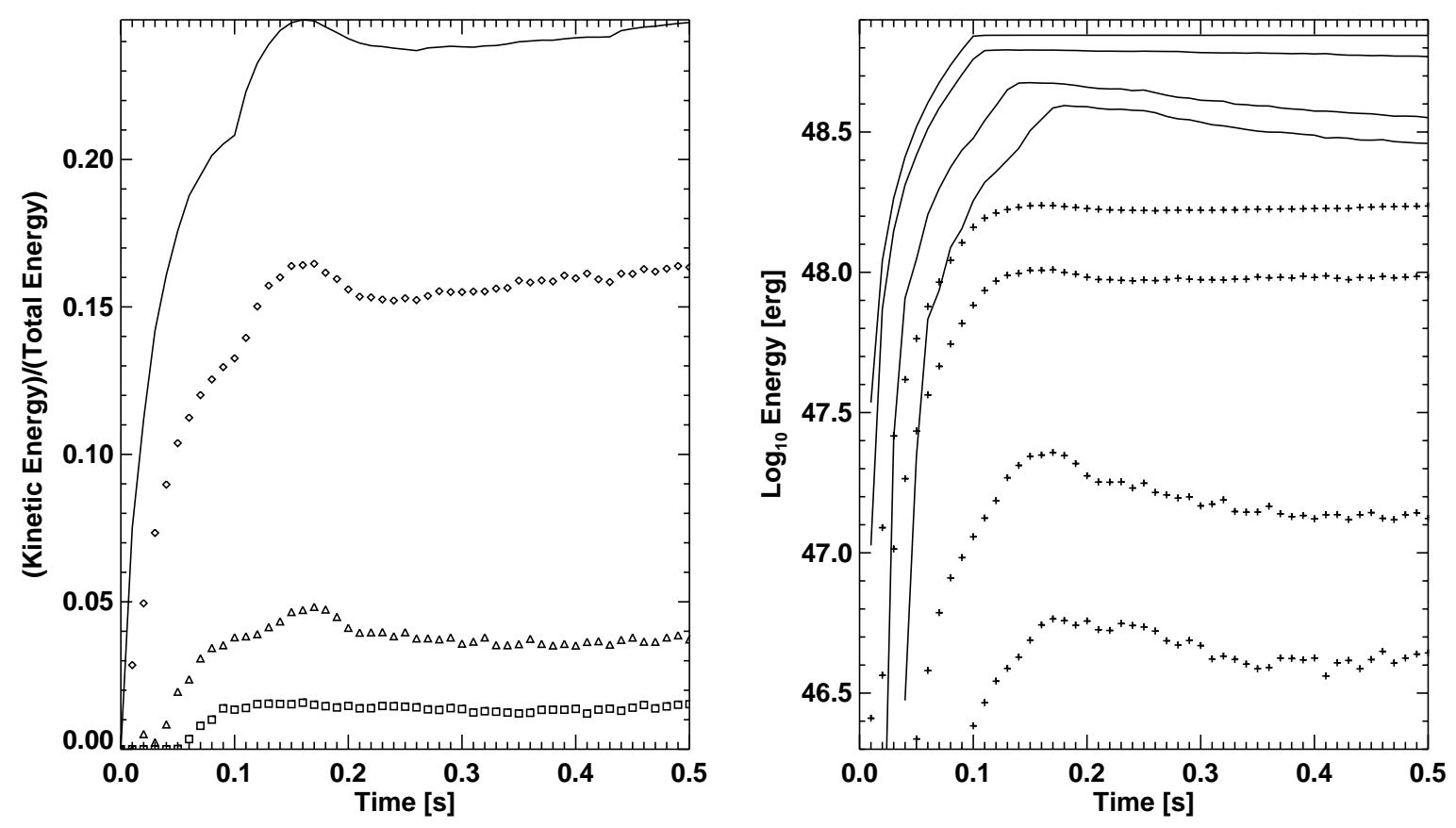

Fig. 31. Time evolution of the ratio of kinetic to total (internal plus kinetic) energy (left panel) of relativistic outflow in model B01 with Lorentz factor above 2, 10, 50 and 100, from top to bottom, respectively. The right panel shows the corresponding evolution of the total energy (solid lines) and of the kinetic energy (crosses) in the same order from top to bottom.

occurs in the presence of tangential velocity components at a propagating shock. It is favored by the fact that the surfaces of constant energy deposition deviate from spheres leading to relatively high energy deposition rates at large radii off the symmetry axis. A relativistic shock with large tangential velocities causes the formation of a rarefaction wave that moves slowly from the lateral edges towards the axis of the polar outflow and allows the Lorentz factor in some of our B-models to become about $20 \%$ larger in the rarefied region than near the symmetry axis of the radial flow. At the same time the ultrarelativistic core, where Lorentz factors of several hundred are reached, is kept collimated by a subsonic negative lateral velocity component while only the surrounding, mildly relativistic ejecta exhibit a very slow sideways expansion. Thus we expect the structure of the fireball to be essentially preserved during the subsequent evolution.

- The lateral profile of the radially averaged energy of type-B models shows a central hot core (of $\approx 3^{\circ}-9^{\circ}$ width) of nearly constant value, surrounded by a layer where the radially averaged energy drops proportional to $\theta^{-2}$. This profile arises generically in all our models. The lateral profile of the Lorentz factor displays a more complex structure whose functional form cannot be properly fitted by a $\theta^{-2}$ or Gaussian decay (which is required in the standard structured jet model) mainly because of off-axis maxima of the Lorentz factor. In case these profiles are able to survive until the fireball reaches transparency, the angular energetic profiles of short bursts may be very similar to those of the long bursts, but the Lorentz factor distribution might be more complex.
Despite the coarsening of the computational grid with increasing radius, the fireball could be well followed from the shutdown of the energy source at $t_{\mathrm{sa}}$ (or during the long-time, gradual decay of the energy release, respectively) to the end of our simulations (at $0.5 \mathrm{~s}$ or $5 t_{\mathrm{sa}}$ ) when the fireball reaches a radial extension of about $1.5 \times 10^{10} \mathrm{~cm}$. For discussing gross observational features we therefore feel safe to assume that global fireball properties can be sufficiently reliably extrapolated to much larger distances. Inhomogeneities on angular scales $\theta_{\text {ih }}>$ $\Gamma^{-1}$ should persist and are expected to be smoothed out only after the Lorentz factor has dropped below $\theta_{\text {ih }}^{-1}$ (e.g., Kumar $\&$ Granot 2003). The maximum Lorentz factors in the front of the outflow are higher than 500. In some of the type-B models regions in the fireball with $\Gamma>1000$ are found at the end of our simulations. The terminal value of the Lorentz factor should depend on the ambient density of the merger site. Our results are obtained for a fireball that accelerates into a $r^{-3.4}$ density profile (chosen mainly to overcome numerical problems in the construction of the initial configuration). However, the real situation may be more complex, e.g., a steeper density gradient might connect the torus "surface" (or a small cloud of baryons that surrounds the BH-torus system as the relic of the preceding, violent merger history) with the much lower and almost constant density of the interstellar medium.

With a half-opening angle of $\theta_{\mathrm{j}} \sim 5^{\circ}-10^{\circ}\left(15^{\circ}-25^{\circ}\right)$ the collimated ultrarelativistic outflows of type-B models having $\Gamma \gtrsim 100(\Gamma \gtrsim 10)$ at $t=0.5 \mathrm{~s}$ after the onset of the energy release by the GRB engine, cover a fraction $f_{\Omega}=$ $1-\cos \theta_{\mathrm{j}} \sim 0.4 \%-1.5 \%(<10 \%)$ of the $\mathrm{sky}^{2}$. Assuming equal

\footnotetext{
2 The numbers in brackets correspond to outflow with Lorentz factors $\gtrsim 10$ at $0.5 \mathrm{~s}$, which shows still ongoing acceleration so that much larger Lorentz factors can finally result, cf. Table 2 .
} 
detectability from all directions within the opening angle this fraction implies about 100 (more than 10$)^{2}$ times more events than measured gamma-ray bursts. A rate of short GRBs of about 100 per year therefore requires an event rate per galaxy and year of $10^{-5}\left(f_{\Omega} / 0.01\right)^{-1}\left(N_{\mathrm{g}} / 10^{9}\right)^{-1}$ where $N_{\mathrm{g}}$ is the number of visible galaxies. Comparing with estimated NS+NS and $\mathrm{NS}+\mathrm{BH}$ merger rates, which are typically around $10^{-5}$ per year and galaxy (with about a factor of 10 or more uncertainty; e.g., Kalogera et al. 2004; Fryer et al. 1999), we therefore conclude that a significant fraction of such mergers but probably not all, should produce GRB viable outflows. Of course, our rate estimate is very simplistic and neglects many important effects (e.g., the redshift distribution of mergers) which a more careful analysis must take into account (see the recent paper by Guetta $\&$ Piran 2004). Nevertheless, our numbers are in the ballpark of their results, and the conclusions are similar. Taking our computed jet opening angles for granted (see also Appendix A) the analysis by Guetta \& Piran 2004 would also mean that not all compact binary mergers can produce GRBs. This in fact must even be expected, considering the special requirements for GRB suitable conditions, e.g., a baryon-poor environment around a black hole-torus system. If the merger remnant, for example, does not immediately or not at all collapse to a black hole (as is probably the case when two neutron stars with rather low masses merge, see e.g. Morrison et al. 2004), the rapidly spinning remnant will pollute its environment by a neutrinodriven wind. In this case a situation similar to our type-A models may result, i.e., the system will be enveloped by a dense, extended baryonic halo, and a GRB is disfavored.

The edges of the ultrarelativistic $(\Gamma>100)$ jet core are very sharp in terms of the isotropic equivalent energy $E_{\text {iso }}$. Maximum (terminal) Lorentz factors of the order of 1000 suggest the potential to account for hard GRB spectra. Our simulations actually showed inhomogeneous and anisotropic, collimated outflows with lateral variation of the Lorentz factor and of the apparent isotropic energy. Hence, we do not expect equal observability from all positions in the beam direction. The maximum values of the apparent isotropic energy, $E_{\mathrm{iso}}$, are found to be up to about $10^{51} \mathrm{erg}$ at angles $\lesssim 10^{\circ}$ around the symmetry axis of the ultrarelativistic outflow, declining towards the outer wings of less relativistic ejecta. These numbers are obtained for an energy deposition rate of a few $10^{50} \mathrm{erg} \mathrm{s}^{-1}$ over a period of typically $100 \mathrm{~ms}$. These are reasonable and not extreme values in view of model calculations for the energy release by neutrino-antineutrinoannihilation in case of post-merging BH accretion (Ruffert \& Janka 1999; Janka et al. 1999; Setiawan et al. 2004). Provided a major fraction of the energy of the ultrarelativistic fireball gets converted to gamma-rays, our maximum isotropic equivalent energies are in good agreement with estimates based on a comparison of the energetics of short and long GRBs, suggesting an approximate fluence-duration proportionality (Balázs et al. 2003). Since long bursts last typically about 50-100 times longer, a similar luminosity (e.g., Mao et al. 1994; $L_{\text {iso }}^{\text {short }} \sim$ $L_{\text {iso }}^{\text {long }} \sim 10^{51-52} \mathrm{erg} \mathrm{s}^{-1}$ ) implies an apparent energy which is around $10^{51} \mathrm{erg}$ for short bursts instead of $\sim 10^{53} \mathrm{erg}$ for long ones (Frail et al. 1997).
Due to the lateral structure of the outflow we also expect some degree of variability between observed bursts, depending on the viewing angle relative to the system axis. Such differences will be superimposed on variations due to intrinsic properties of the binary systems and remnant BH-torus systems, e.g., associated with different masses and spins of the merging neutron stars or black holes. This finding should be taken into account in studies of the diversity of short gamma-ray bursts like the recent one by Rosswog \& Ramirez-Ruiz (2003). These authors also employed the assumption that the ultrarelativistic outflow is confined as suggested by Levinson \& Eichler (2000). Our models, however, show a much different hydrodynamic scenario for the fireball evolution and collimation, in which the outflow-torus interaction, relativistic shock effects, and Kelvin-Helmholtz instabilities play a crucial role. We therefore emphasize that conclusions based on grounds of the simplified picture developed by Levinson \& Eichler (2000) should be drawn with caution. Finally, we repeat that it is impossible to estimate the energetics of ultrarelativistic outflows without performing simulations that follow the complex hydrodynamics phenomena which develop in response to the deposition of energy in the vicinity of the BH-torus system. A static analysis of time slices for mass distribution and energy deposition by $v \bar{v}$-annihilation (e.g., Rosswog \& Ramirez-Ruiz 2002) can therefore be misleading, in particular with respect to the outflow energetics and asymptotic Lorentz factor which are crucial for judging the viability of $v \bar{v}$-annihilation for powering GRBs.

Our simulations are only a preliminary step towards fully self-consistent models. So far we have investigated the relativistic hydrodynamic flow that is triggered by the deposition of energy near the BH-torus system. However, this energy release was prescribed according to a defined functional behavior instead of being linked to the neutrino emission of the evolving accretion torus. We are in the process of removing this limitation by preparing two-dimensional relativistic simulations of the viscosity-driven evolution of $\mathrm{BH}$-torus systems with a simplified, but consistent treatment of neutrino transport and $v \bar{v}$-annihilation.

Acknowledgements. We thank M. Ruffert for providing us with data to construct our initial models. This work was supported by the Sonderforschungsbereich 375 "AstroTeilchenphysik" and the Sonderforschungsbereich-Transregio 7 "Gravitationswellenastronomie" of the Deutsche Forschungsgemeinschaft. M.A.A. acknowledges the partial support of the Spanish Ministerio de Ciencia y Tecnología (AYA2001-3490-C02C01) and the possibility of making use of the SGI-Altix computer of the University of Valencia.

\section{Appendix A: Dependence of the results on the numerical resolution}

The grid resolution chosen for our numerical models is the result of a compromise between computation time and accuracy. The values of $\left(r_{i+1}-r_{i}\right) / r_{i}$ (where $i=1, \ldots, N_{r}, N_{r}$ is the number of grid points in the radial direction and $r_{i}$ the radial position of the center of cell $i$ ) are smaller than 2\% (typically $\sim 1.3 \%$ ) for $N_{r}=500$. This is usually sufficient for problems of astrophysical interest. We have performed a sequence 


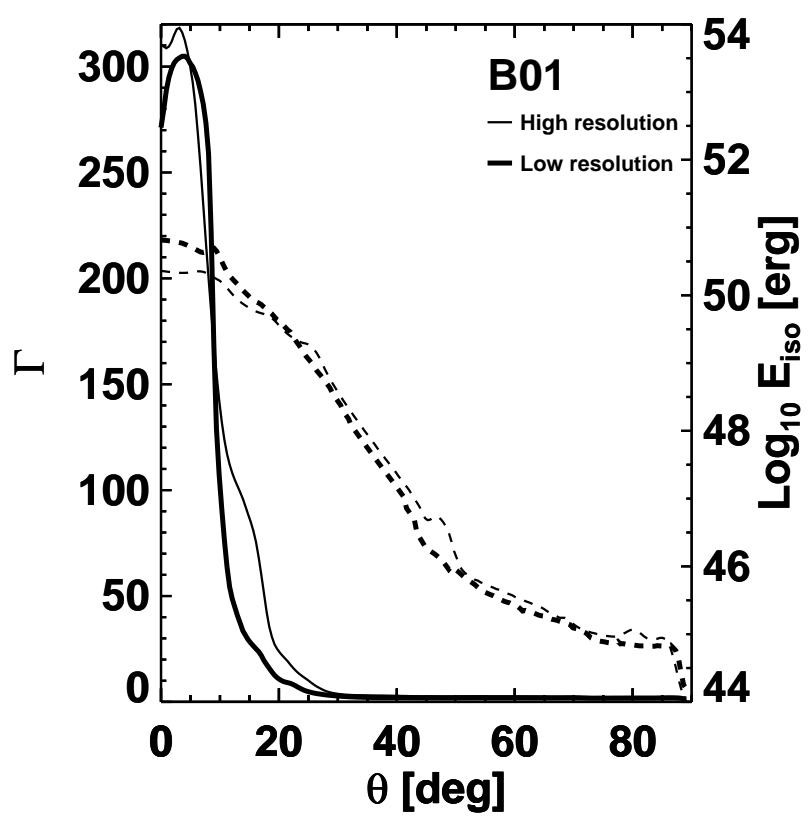

Fig. A.1. Radially averaged values of the Lorentz factor (solid lines) and isotropic equivalent total energies (dashed lines) for model B01 as functions of the polar angle $\theta$ after $0.5 \mathrm{~s}$ of evolution. The thick lines show our standard case computed with 500 radial zones, the thin lines correspond to a simulation with 2000 radial cells.

of tests with larger numbers of zones in the radial direction (up to $N_{r}=2000$, logarithmically spaced). These runs showed more fine structure, in particular at distances $r>3 \times 10^{9} \mathrm{~cm}$. Moreover, a broadening of the forward shock, which is caused by numerical diffusion on the coarser grid at large radii and leads to slightly superluminal propagation, is clearly reduced when the grid has higher resolution. Comparing results for model B01 with $N_{r}=500$ and $N_{r}=2000$ radial zones we find that this numerical artifact has disappeared in the latter case.

Despite of quantitative differences in detail, the two simulations show the same qualitative features (with respect to, e.g., formation, morphology, and dynamics of the outflow, the stretching of the fireball with time, etc.) and agree satisfactorily well in important global properties. Figure A.1 displays lateral profiles of the radially averaged Lorentz factor and of the isotropic equivalent total energy for both cases. The angular width of the ultrarelativistic jet core is nearly the same. As expected, the simulation with the higher resolution reveals more fine structure in the Kelvin-Helmholtz shear layer along the lateral boundary of the ultrarelativistic jet, which induces some differences of the Lorentz factor mainly in the jet wings (below a value of $\Gamma \sim 100$ ). The maximum Lorentz factors are very similar, despite the fact that the better resolved simulation shows less mass entrainment by the jet. This, however, is essentially compensated by a somewhat reduced outflow energy, which manifests itself in a lower (roughly by a factor of 2) maximum of the total isotropic equivalent energy (Fig. A.1). Most other numbers agree on the $10 \%$ level. This supports our confidence in the results obtained with a standard resolution of 500 radial zones. The latter resolution appears as a reasonable compromise for the numerical accuracy considering the approximative nature of our present simulations with respect to employed modeling assumptions, e.g., the setup of the initial model, prescription of the energy release around the black hole, and treatment of the torus evolution without viscous dissipation.

\section{References}

Aloy, M., Martí, J., Gómez, J., et al. 2003, ApJ, 585, L109

Aloy, M. A., Gómez, J.-L., Ibáñez, J. M., Martí, J. M., \& Müller, E. 2000, ApJ, 528, L85

Aloy, M. A., Ibáñez, J. M., Martí, J. M., \& Müller, E. 1999, ApJS, 122,151

Aloy, M. A., \& Martí, J. M. 2002, in Relativistic Flows in Astrophysics, LNP, 589, 197

Balázs, L., Bagoly, Z., Horváth, I., Mészáros, A., \& Mészáros, P. 2003, A\&A, 401, 129

Birkinshaw, M. 1991, MNRAS, 252, 505

Blandford, R. D., \& Znajek, R. L. 1977, MNRAS, 179, 433

Bremer, M., Krichbaum, T. P., Galama, T. J., et al. 1998, A\&A, 332, L13

Cavallo, G., \& Rees, M. J. 1978, MNRAS, 183, 359

Costa, E., Frontera, F., Heise, J., et al. 1997, Nature, 387, 783

Di Matteo, T., Perna, R., \& Narayan, R. 2002, ApJ, 579, 706

Drenkhahn, G. 2002, A\&A, 387, 714

Drenkhahn, G., \& Spruit, H. C. 2002, A\&A, 391, 1141

Duez, M. D., Liu, Y. T., Shapiro, S. L., \& Stephens, B. C. 2004, PRD, 69, 104030

Duncan, R. C., Shapiro, S. L., \& Wasserman, I. 1986, ApJ, 309, 141

Eichler, D., Livio, M., Piran, T., \& Schramm, D. N. 1989, Nature, 340, 126

Font, J. A., \& Daigne, F. 2002, MNRAS, 334, 383

Frail, D. A., Kulkarni, S. R., Nicastro, S. R., Feroci, M., \& Taylor, G. B. 1997, Nature, 389, 261

Fryer, C. L., Woosley, S. E., \& Hartmann, D. H. 1999, ApJ, 526, 152

Galama, T. J., Vreeswijk, P. M., van Paradijs, J., et al. 1998, Nature, 395,670

Goodman, J. 1986, ApJ, 308, L47

Guetta, D., \& Piran, T. 2004, ArXiv Astrophysics e-prints

Hjorth, J., Sollerman, J., Møller, P., et al. 2003, Nature, 423, 847

Janka, H.-T., Eberl, T., Ruffert, M., \& Fryer, C. L. 1999, ApJ, 527, L39

Janka, H.-T., \& Ruffert, M. 2002, in Stellar Collisions, Mergers and their Consequences, ed. M. M. Shara, ASP Conf. Ser., 263, 333

Kalogera, V., Kim, C., Lorimer, D. R., et al. 2004, ApJ, 601, L179

Kohri, K., \& Mineshige, S. 2002, ApJ, 577, 311

Kouveliotou, C., Meegan, C. A., Fishman, G. J., et al. 1993, ApJ, 413, L101

Kumar, P., \& Granot, J. 2003, ApJ, 591, 1075

Lee, W. H., \& Ramirez-Ruiz, E. 2002, ApJ, 577, 893

Levinson, A., \& Eichler, D. 2000, PRL, 85, 236

MacFadyen, A., \& Woosley, S. E. 1999, ApJ, 524, 262

MacFadyen, A., Woosley, S. E., \& Heger, A. 2001, ApJ, 550, 410

Mao, S., Narayan, R., \& Piran, T. 1994, ApJ, 420, 171

Mészáros, P. 2002, ARA\&A, 40, 137

Mizuno, Y., Yamada, S., Koide, S., \& Shibata, K. 2004, ApJ, 606, 395

Mochkovitch, R., Hernanz, M., Isern, J., \& Martin, X. 1993, Nature, 361,236

Morrison, I. A., Baumgarte, T. W., \& Shapiro, S. L. 2004, ApJ, 610, 941

Narayan, R., Piran, T., \& Kumar, P. 2001, ApJ, 557, 949 
Oechslin, R., Uryū, K., \& Thielemann, F. K. 2004, MNRAS, 349, 1469

Pacyński, B. 1986, ApJ, 308, L43

Pacyński, B., \& Wiita, P. J. 1980, A\&A, 88, 23

Piran, T. 1999, Phys. Rep., 314, 575

Piran, T. 2000, Phys. Rep., 333, 529

Piro, L., Heise, J., Jager, R., et al. 1998, A\&A, 329, 906

Pons, J. A., Martí, J. M., \& Müller, E. 2000, J. Fluid Mech., 422, 125

Popham, R., Woosley, S. E., \& Fryer, C. 1999, ApJ, 518, 356

Proga, D., MacFadyen, A. I., Armitage, P. J., \& Begelman, M. C. 2003, ApJ, 599, L5

Qian, Y.-Z., \& Woosley, S. E. 1996, ApJ, 471, 331

Rezolla, L., \& Zanotti, O. 2002, PRL, 114, 501

Rosswog, S., \& Liebendörfer, M. 2003, MNRAS, 342, 673

Rosswog, S., \& Ramirez-Ruiz, E. 2002, MNRAS, 336, 7

Rosswog, S., \& Ramirez-Ruiz, E. 2003, MNRAS, 343, L36

Rosswog, S., Ramirez-Ruiz, E., \& Davies, M. B. 2003, MNRAS, 345, 1077

Rosswog, S., Speith, R., \& Wynn, G. A. 2004, MNRAS, 345, MNRAS, accepted [arXiv: astro-ph/0403500]
Ruffert, M., \& Janka, H.-T. 1999, A\&A, 344, 573

Ruffert, M., \& Janka, H.-T. 2001, A\&A, 380, 544

Ruffert, M., Janka, H.-T., \& Schäfer, G. 1996a, A\&A, 311, 532

Ruffert, M., Janka, H.-T., Takahashi, K., \& Schäfer, G. 1996b, A\&A, 319,122

Salmonson, J. 2003, ApJ, 592, 1002

Sari, R., \& Piran, T. 1997, ApJ, 485, 270

Setiawan, S., Ruffert, M., \& Janka, H.-T. 2004, MNRAS, 352, 753

Shibata, M., Taniguchi, K., \& Uryū, K. 2003, PRD, 68, 084020

Shibata, M., \& Uryū, K. 2000, PRD, 61, 064001

Stanek, K. Z., Matheson, T., Garnavich, P. M., et al. 2003, ApJ, 591, L17

Thompson, T. A., Burrows, A., \& Meyer, B. S. 2001, ApJ, 562, 887 van Paradijs, J., Groot, P. J., Galama, T., Kouveliotou, C., \& Strom, R. G. 1997, Nature, 386, 686

Witti, J., Janka, H.-T., \& Takahashi, K. 1994, A\&A, 286, 841

Woosley, S. E. 1993, ApJ, 405, 273

Zhang, W., Woosley, S. E., \& MacFadyen, A. I. 2003, ApJ, 586, 356 